\title{
A Generalised Fractional Differencing Bootstrap for Long Memory Processes*
}

\author{
George Kapetanios \\ King's Business School, UK \\ Data Analytics for Finance and Macro Research Centre \\ Fotis Papailias ${ }^{\dagger}$ \\ King's Business School, UK \\ Data Analytics for Finance and Macro Research Centre \\ Quantf Research
}

A. M. Robert Taylor

Essex Business School, University of Essex, UK

\begin{abstract}
A bootstrap methodology, first proposed in a restricted form by Kapetanios and Papailias (2011), suitable for use with stationary and nonstationary fractionally integrated time series is further developed in this paper. The resampling algorithm involves estimating the degree of fractional integration, applying the fractional differencing operator, resampling the resulting approximation to the underlying short memory series and, finally, cumulating to obtain a resample of the original fractionally integrated process. While a similar approach based on differencing has been independently proposed in the literature for stationary fractionally integrated processes using the sieve bootstrap by Poskitt, Grose and Martin (2015), we extend it to allow for general bootstrap schemes including blockwise bootstraps. Further, we show that it can also be validly used for nonstationary fractionally integrated processes. We establish asymptotic validity results for the general method and provide simulation evidence which highlights a number of favourable aspects of its finite sample performance, relative to other commonly used bootstrap methods.
\end{abstract}

JEL Codes: C15, C22, C63.

Keywords: Fractional Differencing Bootstrap, Fractional Integration, Resampling.

${ }^{*}$ We thank both the Guest Editor and two anonymous referees for their helpful and constructive comments on earlier versions of the paper.

†Correspondence to: Fotis Papailias, King's Business School, King's College London, Bush House, 30 Aldwych, WC2B 4BG, UK. E-mail Address: fotis.papailias@kcl.ac.uk 


\section{Introduction}

Since the seminal paper by Efron (1979), the bootstrap has rapidly increased in popularity. Initial developments in the bootstrap literature assumed the sample observations to be independent and identically distributed (i.i.d.) and implementation proceeded by randomly resampling single observations with replacement. This approach is, however, inappropriate when dealing with dependent samples. The dependence pattern in the original data is highly unlikely to be preserved and, hence, the bootstrap resamples do not, in general, replicate the key properties of the original series. Consequently, an extensive literature has developed on the use of bootstrap methods for weakly autocorrelated (short memory) series; see, among others, Carlstein (1986), Künsch (1989), Kreiss (1992), Politis and Romano (1992), Politis and Romano (1994) and Bühlmann (1997).

Within the literature two main approaches have been developed for applying bootstrap methods to weakly autocorrelated data: namely, block bootstrap methods and residual-based resampling methods. The idea underlying all block bootstrap approaches is to resample blocks of temporally contiguous observations rather than resampling single observations. For a weakly autocorrelated process the blocks of observations should be approximately independent in large samples and the joint distribution of the variables in different blocks should be approximately the same across blocks. A variety of different block resampling methods has been proposed; among other aspects, the blocks can be non-overlapping or moving, and they can have fixed or variable block length. Block structure optimality and data-driven block length analysis are further considered by Hall, Horowitz and Jing (1995), Bühlmann and Künsch (1999), Politis and White (2004) and Patton, Politis and White (2009), among others. In contrast, residual-based bootstrap approaches are based on the principle of estimating a model and obtaining the residuals. A residual resampling device can be obtained, for example, using i.i.d. random resampling. The estimated parameters from the model fitted to the original data and the residual resample are then used to generate the corresponding resample of the original series.

The above methods were developed for weakly autocorrelated series. In the case of processes which exhibit long range dependence, Lahiri (1993) shows that the block bootstrap is not in general valid even when large block lengths are used. ${ }^{1}$ The residual-based approach, referred to as a sieve autoregressive bootstrap, which obtains the residuals from fitting a long autoregression to the data, is asymptotically valid for fractionally integrated processes whose order of integration is less than one-half (the usual weak stationarity boundary). For relevant such methods in the time domain see, for example, Kapetanios and Psaradakis (2006), Kapetanios (2010), Poskitt (2008) and Poskitt, Grose and Martin (2015).

\footnotetext{
${ }^{1}$ Kim and Nordman (2011) consider the application of block bootstrap-based methods for approximating the sample mean. The resampling is performed on the original long memory series and the normalised bootstrap sample mean is inflated by a factor which depends on the block length and the long memory parameter. This method cannot, however, be used in approximating the distribution of other statistics.
} 
In light of these issues, it is important to develop further bootstrap methods which are asymptotically valid for series which display long range dependence. In this paper we consider an approach to resampling which allows the practitioner to validly implement blockwise and residual-based bootstrap methods designed for weakly autocorrelated with, possibly nonstationary, fractionally integrated series. The procedure can be summarised as follows. Suppose we have a fractionally integrated series. We first use a consistent method to estimate the fractional integration parameter for the series in hand. We then apply the fractional differencing operator appropriate to this estimated parameter, to deliver an approximately short memory series. Established block or residual-based resampling methods are then implemented on this series. Finally, the fractional cumulating operator is applied to the resampled series, using the estimate of the fractional integration parameter obtained from the data. This yields the corresponding resample of the original data. Bootstrap approximations to the distribution of statistics formed from the original data can then be obtained in the usual way. We detail the asymptotic validity of this approach for both stationary and nonstationary fractionally integrated processes. The finite sample performance of our proposed bootstrap procedures is evaluated using a statistic based on the unconditional mean (for stationary fractionally integrated series) and a statistic based on the fractional exponent. We compare it to other widely used block and residual-based bootstrap methods, both where the latter are asymptotically valid and where they are not.

It should at this point be acknowledged that the idea of "pre-filtering" the time series by fractional differencing based on an estimate of fractional integration parameter is not new to the literature. Kapetanios (2010), Kapetanios and Papailias (2011), Papailias and Dias (2015) and Poskitt, Grose and Martin (2015) have all previously adopted this approach in various contexts. In particular, the research in this paper extends the work of Kapetanios and Papailias (2011), and supplements Poskitt, Grose and Martin (2015) by establishing the asymptotic validity of this resampling method when used in conjunction with general bootstrap methodologies, including block bootstrap approaches, for both stationary and nonstationary fractionally integrated series.

The remainder of the paper is organised as follows. Section 2 introduces the fractionally integrated model and the assumptions we will work under, and briefly reviews some semiparametric estimation methods for this model. Here we also review the block, sieve autoregressive and spectral-density-driven bootstrap approaches. Section 3 presents the proposed generalised bootstrap methodology for fractionally integrated series and establishes its asymptotic validity. Section 4 details the results of our simulation experiments into the finite sample behaviour of our approach, relative to existing methods, for the case of bootstrapping a statistic based on the unconditional mean and a statistic based on the fractional exponent. Section 5 concludes. Supporting material, including a proof of our main theoretical result, details of our simulation methodology and additional simulation results, is contained in an accompanying on-line supplementary appendix. 


\section{Preliminaries}

\subsection{Fractional Integration and Semiparametric Estimation}

We consider the fractionally integrated process $Y_{t}$ generated by,

$$
Y_{t}=\Delta_{+}^{-d} u_{t}, \quad t=1,2, \ldots
$$

where the operator $\Delta_{+}^{-d}$, for a generic variable $y_{t}$, is given by $\Delta_{+}^{-d}:=\Delta^{-d} y_{t} \mathbb{I}(t \geq 1)=$ $\sum_{n=0}^{t-1} \pi_{n}(d) y_{t-n}, \mathbb{I}(\cdot)$ denoting the indicator function and $\pi_{n}(d):=\frac{\Gamma(n+d)}{\Gamma(d) \Gamma(n+1)}=\frac{d(d+1) \ldots(d+n-1)}{n !}$ the coefficients in the binomial expansion of $(1-z)^{-d}$. To ensure that $Y_{t}$ is uniquely integrated of order $d$, denoted $I(d)$, we need to define $u_{t}$ in (1) to be a short memory, or $I(0)$, process. This we do by assuming that $u_{t}$ satisfies the following assumption.

Assumption 1. The shocks $u_{t}$ are generated according to the infinite-order moving average $[M A], u_{t}=\varepsilon_{t}+\sum_{j=1}^{\infty} c_{j} \varepsilon_{t-j}$, which satisfies the following conditions:

(a) The MA polynomial $C(z):=1+\sum_{j=1}^{\infty} c_{j} z^{j}$ is such that $C(z) \neq 0,|z| \leq 1$, and $\sum_{j=1}^{\infty} j\left|c_{j}\right|<\infty$.

(b) The innovation process $\varepsilon_{t}$ is ergodic and is such that $E\left(\varepsilon_{t} \mid F_{t-1}\right)=0$ and $E\left(\varepsilon_{t}^{2} \mid F_{t-1}\right)=$ $\sigma_{\varepsilon}^{2}>0$, where $F_{t}$ denotes the $\sigma$-algebra of events determined by $\varepsilon_{s}, s \leq t$. Furthermore, $E\left(\varepsilon_{t}^{4}\right)<\infty$.

Remark 1. Assumption 1 ensures that $u_{t}$ is an $I(0)$ series driven by martingale difference innovations. Under Assumption 1, $u_{t}$ possesses a spectral density which is both finite and bounded away from zero everywhere in the interval $[0, \pi]$. This rules out the possibility of any root at unity in $C(z)$ cancelling with $(1-z)^{d}$, and so $Y_{t}$ is an $I(d)$ process within a wide class of linear processes, including the popular ARFIMA family of models. Under Assumption 1, $u_{t}$ also admits the Autoregressive $[\mathrm{AR}]$ representation $u_{t}=\sum_{j=1}^{\infty} \alpha_{j} u_{t-j}+\varepsilon_{t}$ with $\sum_{j=1}^{\infty}\left|\alpha_{j}\right|<$ $\infty$. Assumption 1 is standard in the sieve autoregressive bootstrap literature and we have therefore chosen to adopt this as our base assumption on $u_{t}$. However, for the other possible bootstrap methods discussed in Section 2.2, additional regularity conditions may be needed on $u_{t}$ to ensure bootstrap validity; we will direct the reader to relevant papers for the precise conditions needed, rather than reproducing them here.

Remark 2. The model in (1) is of so-called "Type II" fractional integration. This type allows the same definition to be valid for any value of the fractional parameter, $d$, and therefore allows for both weakly stationary and nonstationary time series.

A great many papers have appeared in the literature addressing parametric and semiparametric estimation of the parameters of fractionally integrated models. Maximum likelihood 
and pseudo-maximum likelihood approaches are developed in, among others, Fox and Taqqu (1986), Sowell (1992), Hosoya (1997) Robinson (2006), Johansen and Nielsen (2010), Hualde and Robinson (2011), Johansen and Nielsen (2012), Nielsen (2015), Johansen and Nielsen (2016) and Cavaliere, Nielsen and Taylor (2017), among others. Geweke and Porter-Hudak (1983) first considered the semiparametric estimation of the long memory parameter in the frequency domain. Further work on semiparametric estimation has been developed in, among others, Robinson (1995), Andrews and Sun (2004), Nielsen (2005), Shimotsu and Phillips (2005), Shimotsu and Phillips (2006), Abadir, Distaso and Giraitis (2007), Haldrup and Nielsen (2007), Frederiksen and Nielsen (2008) and Frederiksen, Nielsen and Nielsen (2012); see also Nielsen and Frederiksen (2005) for a review of estimation methods for fractionally integrated models.

For our purposes we require an estimate of the long memory parameter, $d$, in (1) which is consistent without the need to specify a parametric model for the short memory process, $u_{t}$. To this end, we therefore focus our attention on the semiparametric estimation methods of Robinson (1995) and Shimotsu and Phillips (2005). Each of these estimators solves a minimisation problem of the form $\widehat{d}:=\arg _{d \in\left[d_{1}, d_{2}\right]} \min R(d)$, where $d_{1}$ and $d_{2}$ are the lower and upper bounds of the values for $d$ such that $-\infty<d_{1}<d_{2}<\infty$ and $R(\cdot)$ is the relevant objective function.

Consider the time series $Y_{t}$ with $t=1,2, \ldots, T$ observations. Following Robinson (1995), the Local Whittle $(L W)$ estimator of $d$ is obtained by minimising the objective function,

$$
R^{L W}(d):=\log \left[\frac{1}{m} \sum_{j=1}^{m} \omega_{j}^{2 d} I\left(\omega_{j}\right)\right]-2 d \frac{1}{m} \sum_{j=1}^{m} \log \omega_{j}
$$

with respect to $d$, where $\omega_{j}:=(2 \pi j) / T$ for $j=1,2, \ldots, T$ and $I\left(\omega_{j}\right)$ is the periodogram, defined as $I\left(\omega_{j}\right):=\frac{1}{2 \pi T}\left|\sum_{j=1}^{T} Y_{t} e^{i \omega_{j} t}\right|^{2}$.

Shimotsu and Phillips (2005) propose the Exact Local Whittle (ELW) estimator which uses a "corrected" discrete Fourier transform of the series. The relevant objective function is now given by,

$$
R^{E L W}(d):=\log \left[\frac{1}{m} \sum_{j=1}^{m} I_{\Delta_{+}^{d} Y_{t}}\left(\omega_{j}\right)\right]-2 d \frac{1}{m} \sum_{j=1}^{m} \log \omega_{j}
$$

where $I_{\Delta_{+}^{d} Y_{t}}\left(\omega_{j}\right)$ is the periodogram of the differenced series, $\Delta_{+}^{d} Y_{t}$.

Assumptions A1 to A4 of Robinson (1995) and Assumptions 1 to 5 of Shimotsu and Phillips (2005) are required for consistency of the $L W$ and $E L W$ estimators, respectively. Under Assumptions A1 to A4 in Robinson (1995), $d \in\left[d_{1}, d_{2}\right], y_{t}$ is invertible and might not be strictly stationary and the bandwidth, $m$, tends to infinity but at a slower rate than T. $L W$ is consistent in the stationary region, while the asymptotic theory is discontinuous 
at $d=\frac{3}{4}$ and $d=1$ and the estimator is inconsistent when $d>1$. Assumptions 1 to 5 of Shimotsu and Phillips (2005) are analogous to Assumptions A1 to A4 in Robinson (1995); however Shimotsu and Phillips (2005) impose a stronger assumption on the rate of $m$ and restrict the permissible values such that $d_{2}-d_{1} \leq \frac{9}{2}$. In Section 3 we introduce a high level assumption concerning the consistency of the estimator of $d$ which therefore entails that the required regularity conditions for consistency hold for a given estimator of $d$.

Remark 3. The model in (1) imposes the unconditional mean of $Y_{t}$ to be zero. In practice, one might want to allow for the possibility that $Y_{t}$ has a non-zero unconditional mean. This can be done through generalising (1) to include a constant, viz,

$$
Y_{t}=\mu+\Delta_{+}^{-d} u_{t}, \quad t=1,2, \ldots
$$

Robinson (1995) discusses the case where $Y_{t}$ is generated according to (4), noting that there is no need to correct the periodogram of $Y_{t}$ for the unknown mean, provided $m<\frac{1}{2} T$, and that here the $L W$ estimator of $d$ will still be consistent for $d \in\left(-\frac{1}{2}, 1\right)$. In the case of the $E L W$ estimator, Shimotsu (2010) suggests estimating $\mu$ by the sample mean, $\bar{Y}=T^{-1} \sum_{t=1}^{T} Y_{t}$, and to use the resulting de-meaned data when calculating the right member of (3). The resulting $E L W$ estimator is consistent for $d \in\left(-\frac{1}{2}, 1\right)$ and also asymptotically normal for $d \in\left(-\frac{1}{2}, \frac{3}{4}\right)$. However, as $d$ increases, the magnitude of the estimation error, $\bar{Y}-\mu$, increases. For $d \geq \frac{1}{2}, \bar{Y}$ is not a consistent estimator of $\mu$ and diverges. In such cases, Shimotsu (2010) suggests the use of the first observation as an estimate for $\mu$; that is, $\widehat{\mu}:=Y_{1}$. In this case, $E L W$ is consistent for $d \geq \frac{1}{2}$ and asymptotically normal for $d \in\left[\frac{1}{2}, 2\right)$. Shimotsu (2010) suggests the James-Stein-type estimator of $\mu$ given by,

$$
\widehat{\mu}(d):=w(d) \bar{Y}+(1-w(d)) Y_{1},
$$

where $w(d)$ is a twice continuously differentiable weight function such that $w(d)=1$ for $d \leq \frac{1}{2}$ and $w(d)=0$ for $d \geq \frac{3}{4}$, and to use $Y_{t}-\widehat{\mu}(d)$ in calculating the periodograms in the objective function of $E L W$ in (3).

Remark 4. Johansen and Nielsen (2016) investigate the effect that initialisation (cf. Remark 2) has on the parametric estimation of nonstationary fractionally integrated time series. Taking a process $Y_{t}$ generated according to (4) they conduct the thought experiment that it exists from a point in time $\left(-N_{0}+1\right)$ but we start to observe the series only from a later time, say $t=1$, onwards. They propose splitting the sample into two parts: (i) pseudoinitial values, $1, \ldots, N$, and (ii) pseudo-observed values $N+1, \ldots, N+T$. Then they mitigate the effect of the unobserved initial values by estimating the model using $N+1, \ldots, N+T$ conditional on $1, \ldots, N$. They find that the estimation of the parameter $\mu$ picks up the effect of the initial values even for the choice $N=0$, in which case $Y_{1}=\mu+u_{1}$. 


\subsection{Resampling Procedures}

In this section we briefly review a number of bootstrap algorithms used in the literature with weakly autocorrelated time series; these will be considered in the rest of this paper. In particular, we describe the sieve autoregressive bootstrap of Kreiss (1992) and Bühlmann (1997), the moving blocks bootstrap of Künsch (1989), the stationary bootstrap of Politis and Romano (1994), and the spectral-density-driven bootstrap of Krampe, Kreiss and Paparoditis (2018).

For the purposes of the discussion in this section we detail the algorithms for the case where the are directly applied to $Y_{t}$ where this is a weakly autocorrelated process; i.e. such that $d=0$ in (1). The probability law characterising the data-generating mechanism is denoted by $\mathcal{P}_{\left\{Y_{1}, \ldots, Y_{T}\right\}}$. We consider the statistic of interest $S_{T}=S_{T}\left(Y_{1}, . ., Y_{T}\right)$ where $F_{S_{T}}(\mathbf{s})$ is the distribution function of $S_{T}$ under $\mathcal{P}_{\left\{Y_{1}, \ldots, Y_{T}\right\}}$. Throughout this paper we assume that the corresponding limiting distribution of $S_{T}$, say $F_{S_{\infty}}(\mathbf{s})$, is continuous. Bootstrap procedures are used to approximate $F_{S_{T}}(\mathbf{s})$ by approximating $\mathcal{P}_{\left\{Y_{1}, \ldots, Y_{T}\right\}}$. Further details on these bootstrap procedures, including the precise regularity conditions on the weakly autocorrelated process under consideration and the class of statistics they can be validly applied to under those regularity conditions, can be found in, inter alia, Kreiss (1992), Bühlmann (1997), Künsch (1989), Politis and Romano (1994), and Krampe, Kreiss and Paparoditis (2018), to whom we direct the reader for further details. Extensions of the sieve bootstrap approach to allow for wild rather than i.i.d. resampling can also be found in the recent paper by Fragkeskou and Paparoditis (2018). In Section 3, we subsequently propose a generalised methodology which allows these bootstrap procedures to be applied to $Y_{t}$ when $d \neq 0$, provided the regularity conditions are met by the process $u_{t}$ in (1).

\subsubsection{Sieve Autoregressive Bootstrap}

The sieve autoregressive (AR) bootstrap was introduced by Kreiss (1992) and further analysed by Bühlmann (1997). Consider the weakly autocorrelated process given by $Y_{t}$ in (1) with $d=0$. Under Assumption 1, $Y_{t}$ admits the infinite-order AR representation, $Y_{t}=\sum_{j=1}^{\infty} \alpha_{j} Y_{t-j}+u_{t}$, where $\sum_{j=1}^{\infty}\left|\alpha_{j}\right|<\infty$; for more details see, among others, Bühlmann (1997), Kreiss, Paparoditis and Politis (2011), and Fragkeskou and Paparoditis (2018).

The sieve bootstrap approximates this infinite order AR by a truncated AR whose order is allowed to increase with the sample size. The sieve AR bootstrap algorithm is as follows.

\section{Algorithm 1.}

Step 1. Given the data $Y_{t}, t=1, \ldots, T$, calculate the statistic of interest $S_{T}=S_{T}\left(Y_{1}, . ., Y_{T}\right)$. Using ordinary least squares [OLS] calculate the AR parameter estimates from fitting an $h$-th order $A R$, denoted $A R(h)$, approximation to $Y_{t}$, and let those estimates be denoted by $\widehat{\alpha}:=\left(\widehat{\alpha}_{1}, \ldots, \widehat{\alpha}_{h}\right)^{\prime}$. 
Step 2. Define the corresponding residuals,

$$
\widehat{u}_{t}:=Y_{t}-\sum_{j=1}^{h} \widehat{\alpha}_{j} Y_{t-j},
$$

and the associated residual variance estimate, $\widehat{\sigma}_{h}^{2}$. Using $\widehat{u}_{t}$, calculate the standardised residuals,

$$
\widetilde{u}_{t}:=\frac{\widehat{u}_{t}-\bar{u}}{s_{\bar{u}}}
$$

where $\bar{u}$ and $s_{\bar{u}}$ denote the sample mean and standard deviation of $\widehat{u}_{t}$, respectively.

Step 3. Let $I_{1}, \ldots, I_{T}$ be i.i.d. random variables with discrete uniform distribution on $\{1, \ldots, T\}$. Then, construct a random sample with replacement from $\widetilde{u}_{t}$ denoted by $\widetilde{u}^{*}:=\left(\widetilde{u}_{1}^{*}, \ldots, \widetilde{u}_{T}^{*}\right)^{\prime}=$ $\left(\widetilde{u}_{I_{1}}, \ldots, \widetilde{u}_{I_{T}}\right)^{\prime}$.

Step 4. The sieve AR bootstrap data observations are generated via the recursion,

$$
Y_{t}^{*}=\sum_{j=1}^{h} \widehat{\alpha}_{j} Y_{t-j}^{*}+\widehat{\sigma}_{h} \widetilde{u}_{t}^{*},
$$

initialised at $Y_{1-j}^{*}=Y_{\tau-j+1}, j=1, \ldots, h$ where $\tau$ has a discrete uniform distribution on $\{h, \ldots, T\}$.

Step 5. Next, based on the bootstrap sample data, compute $S_{T}^{*}=S_{T}\left(Y_{1}^{*}, . ., Y_{T}^{*}\right)$, the bootstrap analogue of $S_{T}$.

Step 6. Repeat Steps 3 to 5, to construct B independent bootstrap samples and bootstrap statistics, denoted $Y_{l, T}^{*}$ and $S_{l, T}^{*}, l=1, \ldots, B$, respectively, and collect $S_{B, T}^{*}:=\left(S_{1, T}^{*}, \ldots, S_{B, T}^{*}\right)^{\prime}$. Approximate $F_{S_{T}}(\mathbf{s})$ by the bootstrap distribution function,

$$
\bar{F}_{S_{B, T}^{*}}(\mathbf{s})=\frac{1}{B} \sum_{l=1}^{B} I\left(S_{B, T}^{*} \leq \mathbf{s}\right) .
$$

Remark 5. The distribution of $S_{T}^{*}$ under the probability law induced by the bootstrap, $\mathcal{P}_{\left\{Y_{1}^{*}, \ldots, Y_{T}^{*}\right\}}$, should mimic that of $S_{T}$ under $\mathcal{P}_{\left\{Y_{1}, \ldots, Y_{T}\right\}}$ and, therefore, $F_{S_{T}^{*}}(\mathbf{s})$ should approximate $F_{S_{T}}(\mathbf{s})$ reasonably well provided that $\mathcal{P}_{\left\{Y_{1}, \ldots, Y_{T}\right\}}$ is in some sense close to $\mathcal{P}_{\left\{Y_{1}^{*}, . ., Y_{T}^{*}\right\}}$. As explained in Poskitt (2008), the analytical determination of $F_{S_{T}^{*}}(\mathbf{s})$ is generally intractable but, using the Glivenko-Cantelli theorem, $\bar{F}_{S_{B, T}^{*}}(\mathbf{s})$ converges to $F_{S_{T}^{*}}(\mathbf{s})$ almost surely (a.s.) as $B \rightarrow \infty$ uniformly in s. Consequently, for a sufficiently large number of bootstrap draws, $B$, we can approximate $F_{S_{T}^{*}}(\mathbf{s})$ arbitrarily closely and anticipate that $\bar{F}_{S_{B, T}^{*}}(\mathbf{s})$ also approximates $F_{S_{T}}(\mathbf{s})$ closely, provided that $F_{S_{T}^{*}}(\mathbf{s})$ is sufficiently close to $F_{S_{T}}(\mathbf{s})$. 
Remark 6. The autoregressive order $h$ used in Step 1 of Algorithm 1 needs to grow with the sample size $T$, such that the residuals $\widehat{u}_{t}$ are approximately uncorrelated. Bühlmann (1997) suggests selecting $h$ using Akaike's Information Criterion (AIC). Poskitt, Grose and Martin (2015) also adopt AIC selection of $h$ using a maximum lag length of $H=\left\lfloor(\log T)^{2}\right\rfloor$, where $\lfloor\cdot\rfloor$ denotes the integer part of its argument.

Remark 7. Fragkeskou and Paparoditis (2018) propose an alternative to the i.i.d. resampling scheme used in Step 3 of Algorithm 1, whereby a wild bootstrap is used. This can allow for the presence of unconditional heteroscedasticity in $u_{t}$. In this case the standardisation in (7) is no longer needed. See Cavaliere, Nielsen and Taylor (2017) for recent application of wild bootstrap methods in long memory time series.

Remark 8. Although designed for the short memory case where $d=0$ in (1), Kapetanios and Psaradakis (2006), Poskitt (2008) and Poskitt, Grose and Martin (2015), show that the sieve AR bootstrap, outlined in Algorithm 1, when applied directly to $Y_{t}$ retains asymptotic validity, provided $h$ satisfies the conditions outlined in Remark 6, even when $|d|<\frac{1}{2}$. However, it is not asymptotically valid for $d \geq \frac{1}{2}$.

\subsubsection{Block Resampling}

An alternative bootstrap approach which can account for the presence of weak dependence in the data is the block bootstrap. Blocks of observations of weakly autocorrelated processes should be constructed such that they are approximately independent and the joint distribution of the variables in different blocks should be almost the same. The main difficulty we face here is the choice of an optimal block size which guarantees the above properties. This can be achieved using data-driven methods such as those developed in Hall, Horowitz and Jing (1995), Politis and White (2004) and Patton, Politis and White (2009).

Consider the weakly autocorrelated process, $Y_{t}$ with $d=0$, and a choice for the block size, b. In addition to Assumption 1, we need to assume that $Y_{t}$ is strictly stationary; see, e.g., Politis and Romano (1994). We consider the following two block bootstrap methodologies:

Moving Block Bootstrap [MBB]: Given the data $Y_{t}, t=1, \ldots, T$, calculate the statistic of interest $S_{T}=S_{T}\left(Y_{1}, . ., Y_{T}\right)$. The MBB of Künsch $(1989)$ divides $\left(Y_{1}, \ldots, Y_{T}\right)^{\prime}$ into $M$ overlapping blocks of $b$ observations, with $M:=T-b+1$. Denote the collection of blocks by $\left\{B_{1}, \ldots, B_{M}\right\}$ and let $I_{1}, \ldots, I_{T / b}$ be i.i.d. random variables with discrete uniform distribution on $\{1, \ldots, M\}$. A $M B B$ resample for $Y_{t}$ is then obtained with replacement from the collection of blocks, $Y_{t}^{*}:=\left(B_{I_{1}}, \ldots, B_{I_{T / b}}\right)^{\prime}$. Repeating this procedure, we construct $B$ independent bootstrap samples and bootstrap statistics, denoted $Y_{l, T}^{*}$ and $S_{l, T}^{*}, l=1, \ldots, B$, respectively, 
and collect $S_{B, T}^{*}:=\left(S_{1, T}^{*}, \ldots, S_{B, T}^{*}\right)^{\prime}$. Approximate $F_{S_{T}}(\mathbf{s})$ by the bootstrap distribution function $\bar{F}_{S_{B, T}^{*}}(\mathbf{s})=\frac{1}{B} \sum_{l=1}^{B} I\left(S_{B, T}^{*} \leq \mathbf{s}\right)$.

Stationary Block Bootstrap [SBB]: Given the data $Y_{t}, t=1, \ldots, T$, calculate the statistic of interest $S_{T}=S_{T}\left(Y_{1}, . ., Y_{T}\right)$. The SBB of Politis and Romano (1994) differs from the $\mathrm{MBB}$ in that it uses a random block length for $b$. Let $L_{1}, L_{2}, \ldots$ be a sequence of geometrically distributed i.i.d. random variables, so that the probability of the event $\left\{L_{i}=\eta\right\}$ is $(1-p)^{\eta-1}$ for $\eta=1,2, \ldots$ and $p$ is a fixed number in $[0,1]$. Denote the collection of blocks by $\left\{B_{L_{1}}, B_{L_{2}}, \ldots\right\}$. The indicator $L_{\eta}$ denotes the length of the relevant block. Let $I_{1}, I_{2}, \ldots$ be a sequence of i.i.d. variables which have discrete uniform distributions on $\{1, \ldots, T\}$. Then, a $S B B$ resample for $Y_{t}$ is obtained by random draws with replacement from the collection of blocks, $Y_{t}^{*}:=\left(B_{L_{1}} B_{L_{2}}, \ldots,\right)^{\prime}$ until $t=T$. Repeating this procedure, we construct $B$ independent bootstrap samples and bootstrap statistics, denoted $Y_{l, T}^{*}$ and $S_{l, T}^{*}, l=1, \ldots, B$, respectively, and collect $S_{B, T}^{*}:=\left(S_{1, T}^{*}, \ldots, S_{B, T}^{*}\right)^{\prime}$. Approximate $F_{S_{T}}(\mathbf{s})$ by the bootstrap distribution function $\bar{F}_{S_{B, T}^{*}}(\mathbf{s})=\frac{1}{B} \sum_{l=1}^{B} I\left(S_{B, T}^{*} \leq \mathbf{s}\right)$.

Remark 9. We refer the reader to Künsch (1989) and Politis and Romano (1994) for the precise regularity conditions needed for the application of the MBB and SBB, respectively. Depending on the choice of the bootstrap method and the statistic of interest, additional restrictions on $u_{t}$ over and above those imposed by Assumption 1 may be necessary. For example, for the case of the SBB Politis and Romano (1994) show that $u_{t}$ needs to be strictly stationary and $\alpha$-mixing with coefficients of size $-3(6+\delta) / \delta$ for $\delta>0$ when the statistic of interest is the mean; see Theorems 1 and 2 and Remark 4 in Politis and Romano (1994). $\diamond$

Remark 10. Kim and Nordman (2011) (henceforth KN) suggest a direct application of the usual block bootstraps in long memory series in order to approximate the distribution of the mean. They consider the fractional noise model; hence in (1) they let $u_{t}$ be i.i.d. variables with zero mean and finite variance and allow $|d|<\frac{1}{2}$ but not $d \geq \frac{1}{2}$. They then apply $M B B$ in the usual way. Disregarding the presence of long memory, they split the sample into blocks and randomly resample from these blocks. In order for the resample to successfully mimic the true mean, they "inflate" the statistic multiplying it by $b^{d}$. This methodology, however, cannot be generalised to other statistics and cannot be applied, in general, in nonstationary long memory series; see KN for further details.

\subsubsection{Spectral-Density-Driven Bootstrap}

The sieve and block bootstraps procedures outlined above are both carried out in the time domain. We also consider the spectral-density-driven bootstrap (SDDB) of Krampe, Kreiss 
and Paparoditis (2018). We next provide a brief description of the algorithm. We refer the reader to Krampe, Kreiss and Paparoditis (2018) for further technical details.

Consider the $M A(\infty)$ representation of the weakly autocorrelated process $Y_{t}$ in (1) with $d=0$

$$
Y_{t}=\varepsilon_{t}+\sum_{j=1}^{\infty} c_{j} \varepsilon_{t-j},
$$

and consider an estimator $\widehat{f}_{Y}$ of the spectral density of $Y_{t}, f_{Y}$; see Krampe, Kreiss and Paparoditis (2018) for precise conditions placed on the estimator of $f_{Y}$. Given the statistic of interest $S_{T}=S_{T}\left(Y_{1}, . ., Y_{T}\right)$ evaluated using the original data, the SDDB consists of the following steps.

\section{Algorithm 2.}

Step 1. Compute the Fourier coefficients of $\log \left(\widehat{f}_{Y}\right)$ given by,

$$
\widehat{a}_{k}:=\frac{1}{2 \pi} \int_{0}^{2 \pi} \log \left\{\widehat{f}_{Y}(\omega)\right\} e^{-i k \omega} d \omega
$$

for $k=0,1,2, \ldots$

Step 2. Let $\widehat{\sigma}_{a}^{2}:=2 \pi e^{\widehat{a}_{0}}$ and compute the coefficients $\widehat{c}_{k}$ using,

$$
\widehat{c}_{k+1}=\sum_{j=0}^{k}\left(\frac{1-j}{k+1}\right) \widehat{a}_{k+1-j} \widehat{c}_{j}
$$

for $k=0,1,2, \ldots$ and starting value $\widehat{c}_{0}=1$.

Step 3. Generate i.i.d. pseudo-innovations $\left(\varepsilon_{1}^{*}, \ldots ., \varepsilon_{T}^{*}\right)^{\prime}$ with mean 0 and variance $\widehat{\sigma}_{a}^{2}$.

Step 4. Generate the pseudo-time series $\left(Y_{1}^{*}, \ldots ., Y_{T}^{*}\right)^{\prime}$ obtained by,

$$
Y_{t}^{*}=\sum_{j=0}^{\infty} \widehat{c}_{j} \varepsilon_{t-j}^{*}, \text { for } t=1,2, \ldots, T
$$

Step 5. Next, using the bootstrap data from Step 4, compute $S_{T}^{*}=S_{T}\left(Y_{1}^{*}, . ., Y_{T}^{*}\right)$, the bootstrap analogue of $S_{T}$.

Step 6. Repeat Steps 3 to 5, to construct B independent bootstrap samples and bootstrap statistics, denoted $Y_{l, T}^{*}$ and $S_{l, T}^{*}, l=1, \ldots, B$, respectively, and collect $S_{B, T}^{*}:=\left(S_{1, T}^{*}, \ldots, S_{B, T}^{*}\right)^{\prime}$. Approximate $F_{S_{T}}(\mathbf{s})$ by the bootstrap distribution function $\bar{F}_{S_{B, T}^{*}}(\mathbf{s})=\frac{1}{B} \sum_{l=1}^{B} I\left(S_{B, T}^{*} \leq\right.$ s). 
Remark 11. Algorithm 2 can be modified to use the estimated AR representation instead of the MA representation. In the accompanying on-line supplementary appendix, we discuss how the SDDB using the MA, as well as the AR representations, is implemented in our experiments; for more details see Section 3.1 in Krampe, Kreiss and Paparoditis (2018).

\section{The Fractional Differencing Bootstrap}

In the previous section, we discussed the well-known sieve AR bootstrap, together with the MBB, SBB and SDDB methods for the special case where $Y_{t}$ in (1) is a weakly autocorrelated process such that $d=0$. If we restrict $|d|<\frac{1}{2}$, then the sieve AR bootstrap can be validly applied to the original series $Y_{t}$; see Poskitt (2008). This partially solves the problem of resampling stationary fractionally integrated processes. However, block bootstraps still cannot be used and, furthermore, when there is increased persistence in the series, i.e. $d$ approaches or exceeds the $\frac{1}{2}$ value, the finite sample performance of the sieve AR bootstrap deteriorates; see Figure 1(iv) in Poskitt, Grose and Martin (2015). Poskitt, Grose and Martin (2015) suggest that filtering the original series using an estimate of $d$ can improve the performance of the sieve AR bootstraps; see Figure 3(iv) in Poskitt, Grose and Martin (2015). Nonetheless, the issues of (i) resampling nonstationary fractionally integrated processes $(d \geq$ $\frac{1}{2}$ ), and (ii) applying block bootstraps in stationary and nonstationary fractionally integrated processes still remain.

Remark 12. In an analogous fashion to Remark 4 in Shimotsu and Phillips (2005), we can also define fractionally integrated processes as,

$$
Y_{t}:=\left\{\begin{array}{c}
\Delta_{+}^{-d} u_{t}, d \in\left(-\infty, \frac{1}{2}\right), \\
\sum_{k=1}^{t} Z_{k}, Z_{t}:=\Delta_{+}^{1-d} u_{t}, \quad d \in\left[\frac{1}{2}, \frac{3}{2}\right),
\end{array}\right.
$$

with extensions for larger values of $d$ so that $Y_{t}$ (or its higher-order) difference is stationary. This "difference-and-add-back" approach corresponds to the "unconditional fractional model" discussed in Johansen and Nielsen (2016). Using this definition, we can partially solve the problem of resampling nonstationary fractionally integrated processes because the sieve AR bootstrap can be applied directly to $Y_{t}$ or its higher-order difference. This is also discussed in Poskitt, Grose and Martin (2015). However, other types of bootstrap, such as block resampling, still cannot be used, since the underlying series is not weakly autocorrelated.

In a similar fashion to the "difference-and-add-back" approach, we propose a generalised bootstrap methodology which allows bootstrap methods designed for weakly autocorrelated series to be validly implemented for both stationary and nonstationary long memory series. 
The proposed methodology does not require any range restrictions on $d$ provided that an estimator of $d$ can be found which satisfies the following assumption.

Assumption 2. Suppose that $Y_{t}$ is generated by (1), and let Assumption 1 hold. Then, we assume that there exists an estimator, $\widehat{d}$, of the true fractional integration parameter, $d$, which satisfies the condition that $\operatorname{Var}(\widehat{d}-d)=o(1)$.

Remark 13. Assumption 2 is rather general and does not specify any particular method for estimating $d$. In our context, obvious candidates are the $L W$ and $E L W$ estimators discussed in Section 2. For consistency, the bandwidth, $m$, used for the $L W$ estimator must satisfy the condition that $\frac{1}{m}+\frac{m}{T} \rightarrow 0$ as $T \rightarrow \infty$, while for $E L W$ the required rate on $m$ is that $\frac{1}{m}+\frac{m(\log m)^{\frac{1}{2}}}{T}+\frac{\log T}{m^{\gamma}} \rightarrow 0$ as $T \rightarrow \infty$, for any $\gamma>0$. For $E L W$ the range of permissible values in the optimisation in (3) is such that $d_{2}-d_{1} \leq \frac{9}{2}$, although note that this does not restrict the value of $d$ itself. The full set of required conditions for consistency are given in Assumptions A1 to A4 of Robinson (1995) for the $L W$ estimator and in Assumptions 1 to 5 of Shimotsu and Phillips (2005) for the ELW estimator. Under the above conditions, Assumption 2 holds for both $L W$ and $E L W$ estimators of $d$, although in the case of $L W$ it is important to note that this estimator is consistent for $d<1$ alone.

The Fractional Differencing bootstrap procedure (FDB) we propose in this paper is now detailed in Algorithm 3.

\section{Algorithm 3.}

Step 1. Given the data $Y_{t}, t=1, \ldots, T$, calculate the statistic of interest $S_{T}=S_{T}\left(Y_{1}, . ., Y_{T}\right)$.

Step 2. Obtain an estimate, $\widehat{d}$, of the long memory parameter, d, satisfying Assumption 2.

Step 3. [Differencing] Filter $Y_{t}$ using the fractional differencing operator, $\Delta_{+}^{\widehat{d}}$, to obtain,

$$
\widehat{u}_{t}:=\Delta_{+}^{\widehat{d}} Y_{t}
$$

Step 4. [Resampling] Apply any asymptotically valid bootstrap method for weakly autocorrelated series to obtain a resample from $\widehat{u}_{t}$, denoted by $\widehat{u}_{t}^{*}$.

Step 5. [Cumulation] Apply the fractional cumulating operator, $\Delta_{+}^{-\widehat{d}}$, to $\widehat{u}_{t}^{*}$ and obtain the corresponding fractionally integrated resampled data,

$$
Y_{t}^{*}:=\Delta_{+}^{-\widehat{d}_{u_{t}}^{*}}
$$

Step 6. Calculate $S_{T}^{*}=S_{T}\left(Y_{1}^{*}, . ., Y_{T}^{*}\right)$ which is the bootstrap analogue of $S_{T}$. 
Step 7. Repeat Steps 4 to 6, to construct B independent bootstrap samples and bootstrap statistics, denoted $Y_{l, T}^{*}$ and $S_{l, T}^{*}, l=1, \ldots, B$, respectively, and collect $S_{B, T}^{*}:=\left(S_{1, T}^{*}, \ldots, S_{B, T}^{*}\right)^{\prime}$. Approximate $F_{S_{T}}(\mathbf{s})$ by the bootstrap distribution function $\bar{F}_{S_{B, T}^{*}}(\mathbf{s})=\frac{1}{B} \sum_{l=1}^{B} I\left(S_{B, T}^{*} \leq\right.$ s).

The idea behind the proposed bootstrap methodology detailed in Algorithm 3 is that, under Assumptions 1 and 2, in Step 4 we resample from the fractionally differenced series, $\widehat{u}_{t}$, which, given the consistency of $\hat{d}$, is an approximately weakly dependent, $I(0)$, series, rather than from the levels data $Y_{t}$. We then obtain the corresponding fractionally integrated resample in Step 5. This allows the use of bootstrap methods in Step 4 originally designed to be applied to weakly autocorrelated series. In particular, we can apply any of the sieve AR bootstrap, MBB, SBB, SDDB approaches in Step 4 of Algorithm 3. In doing so we follow the steps for those resampling methods exactly as described in the algorithms of Section 2 . These bootstrap methods are valid, provided that the regularity conditions stated in the original papers, and discussed in Section 2, hold for the $I(0)$ series, $u_{t}$, in (1). The approach detailed in Algorithm 3 can therefore accommodate both stationary and nonstationary fractionally integrated processes, although it should be noted that for the nonstationary case, $d \geq 1 / 2$ in (1), the class of statistics which can be validly bootstrapped using Algorithm 3 is reduced, as discussed below. It is important to recognise that our aim in this paper is to provide a general framework for resampling long memory time series which retains the large sample properties that would be obtained using the same resampling method applied directly to a weakly autocorrelated series with the necessary regularity conditions imposed on the underlying shocks, $u_{t}$.

As mentioned in the Introduction, this paper is not the first to discuss the use of the "differencing" technique based on an estimate of the fractional integration parameter employed in Algorithm 3. Kapetanios (2010), Kapetanios and Papailias (2011), Papailias and Dias (2015) and Poskitt, Grose and Martin (2015) also apply the same method in time series analysis and forecasting. Algorithm 3 extends the work in Kapetanios and Papailias (2011) and shares some similarities with Poskitt, Grose and Martin (2015) who investigate the case of the sieve AR bootstrap. However, we further generalise Poskitt, Grose and Martin (2015) in two aspects: (i) we allow the application of any valid bootstrap for weakly autocorrelated series, including block bootstraps and bootstraps in the frequency domain, and (ii) we do not restrict the value of the long memory parameter, accommodating both stationary and nonstationary series.

A natural question arises about the nature of the statistics under consideration. In what follows, we can apply our approach to statistics which satisfy the following assumption.

Assumption 3. Suppose that $Y_{t}$ is generated by (1) and let Assumption 2 hold. Define $\boldsymbol{Y}_{T}:=\left(Y_{1}, \ldots, Y_{T}\right)^{\prime}$ and let the corresponding bootstrap sample, obtained according to Algorithm 3, be denoted by $\boldsymbol{Y}_{T}^{*}:=\left(Y_{1}^{*}, \ldots, Y_{T}^{*}\right)^{\prime}$. Furthermore, let $\mathcal{N}$ be a compact subset 
of $\mathbb{R}^{T}$. Then for all $\boldsymbol{Y}_{T}, \boldsymbol{Y}_{T}^{*} \in \mathcal{N}$ there exists a family of Borel-measurable functions $B_{t}: \mathbb{R}^{T} \times \mathbb{R}^{T} \rightarrow[0, \infty)$, satisfying:

$$
\lim _{T \rightarrow \infty} \sup T^{-1} \sum_{t=1}^{T} E\left[E^{*}\left[B_{t}\left(\boldsymbol{Y}_{T}, \boldsymbol{Y}_{T}^{*}\right)^{2}\right]\right]<\infty,
$$

for which,

$$
\left\|S_{T}-S_{T}^{*}\right\|^{2} \leq T^{-\left(1+\max \left\{0, d-\frac{1}{2}\right\}\right)} \sum_{t=1}^{T} B_{t}\left(\boldsymbol{Y}_{T}, \boldsymbol{Y}_{T}^{*}\right)\left|Y_{t}-Y_{t}^{*}\right| .
$$

where $E[\cdot]$ and $E^{*}[\cdot]$ denote the expectations with respect to $\mathcal{P}_{\boldsymbol{Y}_{T}}$ and $\mathcal{P}_{\boldsymbol{Y}_{T}^{*}}$, respectively, $S_{T}$ is the statistic calculated on the original data and $S_{T}^{*}$ the bootstrap statistic calculated using the bootstrap data obtained using $\widehat{d}$ for both differencing and cumulation (Steps 3 and 5 in Algorithm 3).

Remark 14. For $d<\frac{1}{2}$ Assumption 3 coincides with Assumption 4 of Poskitt (2008). In this case an upper bound of $T^{-1} \sum_{t=1}^{T} B_{t}\left(\boldsymbol{Y}_{T}, \boldsymbol{Y}_{T}^{*}\right)^{2}\left|Y_{t}-Y_{t}^{*}\right|$ in (10) suffices. Where $Y_{t}$ is nonstationary, this upper bound must be strengthened because the term $\left|Y_{t}-Y_{t}^{*}\right|$ needs to be scaled by $T^{-\max \left\{0, d-\frac{1}{2}\right\}}$ for it to be bounded when $d>\frac{1}{2}$. Therefore, for $0 \leq d \leq \frac{1}{2}$, $T^{-\max \left\{0, d-\frac{1}{2}\right\}}=1$ and so the upper bound in (10) coincides with that given in Assumption 4 of Poskitt (2008). Where $d>\frac{1}{2}$, the scale factor applied to the right member of (10) is, therefore, strengthened from $T^{-1}$ to $T^{-\frac{1}{2}-d}$.

Remark 15. As discussed in Remark 14, Assumption 3 coincides with Assumption 4 of Poskitt (2008) when $d<\frac{1}{2}$. In this case Assumption 3 therefore places exactly the same restrictions on the class of statistics which may be considered as are imposed by Assumption 4 of Poskitt (2008). Lemma 1 of Poskitt (2008) therefore also applies here and characterises the class of statistics allowed. As discussed in Poskitt (2008), these include the sample mean, sample autocorrelations, sample autocovariances and sample partial autocorrelations. The expansions needed to establish the normality of the semiparametric $L W$ and $E L W$ estimates of $d$ discussed in Section 2.1, indicate that Lemma 1 of Poskitt (2008) also holds for these statistics, as the estimators are differentiable functions of sums of functions of observed data with continuous partial derivatives; see Equation (4.2) in Robinson (1995) for the $L W$ estimator and Equation (54) in Shimotsu and Phillips (2005) for the $E L W$ estimator. The conditions imposed by Assumption 3 where $d \geq \frac{1}{2}$ are considerably stronger, however, and no longer allow for statistics such as the sample variance and sample autocorrelations, but do still allow for the $L W$ and $E L W$ estimates although these would of course need to be such that they satisfy the regularity conditions ensuring consistency, discussed in Section 2.1. Such restrictions on the class of statistics allowed is not unique to the methods we discuss in this paper, and similar restrictions apply in general in the fractionally integrated literature on the class of statistics upon which asymptotically valid inference can be conducted when 
$d \geq \frac{1}{2}$. One commonly suggested solution in the literature where it is thought that $d>\frac{1}{2}$, is first to difference the data and then conduct inference on the resulting differenced series. The same approach could be taken here and, provided $d<\frac{3}{2}$, only Assumption 4 of Poskitt (2008) would then need to be satisfied in the differenced series.

Remark 16. Assumption 3 requires that $\boldsymbol{Y}_{T}^{*}$ is obtained using Algorithm 3. It is important to recognise that Step 4 of Algorithm 3 requires that a bootstrap method which is asymptotically valid for use with weakly autocorrelated series is employed. Any of the methods outlined in Section 2.2 can therefore be used, provided the regularity conditions referred to there are met. Specific statements regarding asymptotic behaviour will depend on the bootstrap method being applied to the underlying weakly autocorrelated series and the particular statistic of interest.

We now state our main theorem concerning the large sample validity of the FDB method described in Algorithm 3. A general proof of the result, which covers any of the bootstrap resampling schemes discussed in Section 2.2 and statistics that satisfy Assumption 3, is provided in the accompanying on-line supplementary appendix.

Theorem 1. Let $\eta\left(F_{X}, F_{Y}\right)$ denote the Mallows metric for the distance between two probability distributions $F_{X}$ and $F_{Y}$, defined as inf $\left\{E\|X-Y\|^{2}\right\}^{\frac{1}{2}}$, where the infimum is taken over all square integrable random variables $X$ and $Y$ in $\mathbb{R}$ with marginal distributions $F_{X}$ and $F_{Y}$. Consider any statistic $S_{T}$ which satisfies Assumption 3 and which has a continuous limiting distribution. Then, with probability one,

$$
\eta\left(F_{S_{T}^{*}}, F_{S_{T}}\right) \rightarrow 0 \text { as } T \rightarrow \infty
$$

where $F_{S_{T}}$ is the distribution function of $S_{T}$ under $\mathcal{P}_{\left\{Y_{1}, \ldots, Y_{T}\right\}}$ and $F_{S_{T}^{*}}$ is the distribution of $S_{T}^{*}$ under $\mathcal{P}_{\left\{Y_{1}^{*}, \ldots, Y_{T}^{*}\right\}} \cdot$

Remark 17. For a discussion regarding the properties of $\eta\left(F_{X}, F_{Y}\right)$ see Bickel and Freedman (1981) and Remark 1 of Poskitt (2008). As in Poskitt (2008), from the triangular inequality we have $\eta\left(\bar{F}_{S_{B, T}^{*}}, F_{S_{T}}\right) \leq \eta\left(\bar{F}_{S_{B, T}^{*}}, F_{S_{T}^{*}}\right)+\eta\left(F_{S_{T}^{*}}, F_{S_{T}}\right)$. Because $\eta\left(\bar{F}_{S_{B, T}^{*}}, F_{S_{T}^{*}}\right) \rightarrow$ 0 a.s. (Lemma 8.4 in Bickel and Freedman (1981)), it follows from Theorem 1 that $\eta\left(\bar{F}_{S_{B, T}^{*}}, F_{S_{T}}\right) \rightarrow$ 0 a.s. This validates the use of bootstrap in the scenarios considered in this paper.

Remark 18. Theorem 1 relies on the assumption that the limiting distribution of the statistic of interest, $S_{T}$, is continuous. This rules out degenerate cases, an obvious example being where the statistic of interest was inappropriately scaled in the sample size, $T$, such that $S_{T}$ was of $o_{p}(1)$; the result in Theorem 1 would not be informative in such cases. Where $d<\frac{1}{2}$, the sample mean, sample autocorrelations, and the semiparametric $L W$ and $E L W$ 
estimates of $d$ all satisfy the conditions imposed by Theorem 1, given that they possess asymptotic normal distributions; see Theorem 8 in Hosking (1996) for the normality of the sample mean, Theorem 2 in Robinson (1995) for the normality of the $L W$ estimate and Theorem 2.2 in Shimotsu and Phillips (2005) for the normality of the ELW estimate. Where $d \geq \frac{1}{2}$, statistics such as the sample mean and sample autocorrelations do not satisfy the requirements of Theorem 1; see again the discussion in Remark 15. However, the $L W$ estimate is still covered by Theorem 1 for $d<1$ (excluding $d=\frac{3}{4}$ ), as is the $E L W$ estimate for any value of $d$; see again the discussion in Section 2.1 .

Remark 19. In the bootstrap literature a conventional way to establish bootstrap consistency for weakly autocorrelated processes is to show that the following result holds:

$$
\sup _{x}\left|\mathcal{P}_{\boldsymbol{x}^{*}}\left(S^{*}>x\right)-\mathcal{P}_{\boldsymbol{x}}(S>x)\right|=o_{p}(1)
$$

The use of Mallows' distance in our context, as is also done in Poskitt (2008), can be justified by Lemma 8.3 (b) of Bickel and Freedman (1981) which states that Mallows convergence is equivalent to convergence in distribution and the existence of second moments for the statistic of interest. Since convergence in distribution in the original and bootstrap probability space implies (11) by Pólya's theorem, we obtain a link between Mallows convergence and the general $I(0)$ bootstrap literature.

\section{Simulations}

\subsection{Implementation}

As discussed in Remark 3, the model in (1) assumes that the unconditional mean of $Y_{t}$ is known to be zero. However, in practice it is more reasonable to take the unconditional mean to be unknown and model $Y_{t}$ according to (4). The standard bootstrap algorithms discussed in Section 2 and the FDB Algorithm 3 of Section 3 should therefore be modified accordingly to allow for the estimation of $\mu$. The modified algorithm, Algorithm 4, which we use in the simulation experiments reported here, is detailed in the accompanying on-line supplementary appendix. All experiments are based on $B=199$ bootstrap draws and $R=1000$ Monte Carlo repetitions. ${ }^{2}$ We report results for two statistics based on the mean and the fractional

\footnotetext{
${ }^{2}$ All simulations were performed in $\mathrm{R}$ using the rnorm() random number generator, along with the arima.sim() and ar.ols() routines. The fractionally integrated series were generated using the fracdiff() routine of Jensen and Nielsen (2014), available from http://www. econ.queensu.ca/faculty/mon/software/. For computing the MBB procedures the tsbootstrap() routine from the tseries $\mathrm{R}$ package was used. The b.star() routine from the $n p \mathrm{R}$ package was used to compute the optimal data-dependent block length of Patton, Politis and White (2009). Additional code from Krampe, Kreiss and Paparoditis (2018) available from https://www.tu-braunschweig.de/Medien-DB/stochastik/code-snippet_sddb.txt and Shimotsu and Phillips (2005) available from http://shimotsu.web.fc2.com/Site/Matlab_Codes.html was also used.
} 
differencing parameter of $Y_{t}$. Our aim is to evaluate the finite sample accuracy of the FDB estimate of the distribution of the statistic under consideration and to what extent, if any, it improves upon the finite sample properties of the standard bootstrap methods from Section 2. The statistics we consider are given by,

$$
S_{T}^{\mu}:=T^{\frac{1}{2}-d}(\bar{Y}-\mu) \text { and } S_{T}^{d}:=m^{\frac{1}{2}}(\widehat{d}-d)
$$

where $\bar{Y}$ denotes the sample mean of $Y_{t}$. Analogously, their bootstrap counterparts are,

$$
S_{T}^{\mu *}:=T^{\frac{1}{2}-\widehat{d}}\left(\bar{Y}^{*}-\bar{Y}\right) \text { and } S_{T}^{\widehat{d} *}:=m^{\frac{1}{2}}\left(\widehat{d}^{*}-\widehat{d}\right)
$$

where $\bar{Y}^{*}$ and $\widehat{d}^{*}$ denote the sample mean and estimated long memory parameter (the latter using the same estimator as for the original data) calculated from the bootstrap data. We report empirical $90 \%$ coverage rates using the relevant bootstrap quantiles for each statistic, $q_{0.05}^{*}$ and $q_{0.95}^{*}$, where $q_{\alpha}^{*}$ denotes the $100 \alpha \%$ bootstrap empirical quantile.

Our simulation DGP for $Y_{t}$ is the $\operatorname{ARFIMA}(1, d, 1)$ model given by $Y_{t}=\mu+\Delta_{+}^{-d} u_{t}$, $t=1,2, \ldots, T$, setting $\mu=0$ in the DGP without loss of generality. Results are reported for samples of size $T=120$ and $T=500$. As mentioned above, we account for $\mu$ in the estimation of $d$, applying $L W$ and $E L W$ to the demeaned series $Y_{t}-\widehat{\mu}(d)$, with $\widehat{\mu}(d)$ as defined in (5). The bandwidth $m$ used in connection with these estimators was chosen according to the popular rule-of-thumb $m:=\left\lfloor T^{1 / 2}\right\rfloor$. Results are reported for $d \in\{0,0.15,0.25,0.35,0.45,0.49,0.75\}$, with $d=0.75$ omitted in the case of the results for bootstrapping the mean because the sample mean does not provide a consistent estimate of $\mu$ in this case. The disturbances $u_{t}$ were generated according to the $A R M A(1,1)$ process, $u_{t}=\phi u_{t-1}+\varepsilon_{t}+\theta \varepsilon_{t-1}$ with $\varepsilon \sim \operatorname{NIID}(0,1)$ and $u_{t}=\varepsilon_{t}=0, t \leq 0$. Results are reported for $\phi \in\{0,0.8,-0.8\}$ and $\theta \in\{0,0.8,-0.8\}$. By including: (i) a large positive value of $\phi$, and (ii) a large negative value of $\theta$, in the range of simulation DGPs considered we have included cases where: (i) there are two separate sources of significant persistence in the time series arising through both positive fractional integration, $d>0$, and relatively high persistence in the underlying weakly autocorrelated series, and (ii) we have a positively fractionally integrated process driven by large negative moving average shocks. Both of these scenarios are known to be extremely challenging from the perspective of obtaining reliable inference in finite samples, for both bootstrap and asymptotic-based methods.

The bootstrap procedures we include are:

- The $M B B$ and $S B B$ methods applied directly to the original series using the following block length choices: $b_{1}:=T^{1 / 5}, b_{2}:=T^{1 / 4}$ and $b_{3}:=T^{1 / 3}$, as suggested by Hall, Horowitz and Jing (1995) Additionally, we also use a data-dependent block length, denoted by $b^{*}$, of the form given in Patton, Politis and White (2009). We will use $b^{*}$ for both $S B B$ and $M B B$. 
- The KN versions of the $M B B$ and $S B B$ methods applied directly to the original series using the following block length choices: $b_{4}:=\frac{1}{2} T^{1 / 2}, b_{5}:=T^{1 / 2}$ and $b_{6}:=2 T^{1 / 2}$, as suggested by KN.

- The SDDB method using both the AR and MA representations.

- The sieve AR bootstrap method with two choices for $h$ : (i) using AIC with a maximum lag order of $H:=\left\lfloor(\log T)^{2}\right\rfloor$, and (ii) $h=H$ which therefore results in a fixed-length long autoregressive order.

- The FDB versions of the $M B B, S B B, S D D B$ using either the AR or MA representation, and sieve AR bootstrap methods.

Algorithm 5 in the accompanying on-line supplementary appendix provides technical details concerning the simulation design and the calculation of coverage rates. We implement Algorithms 4 and 5 using the $L W$ and $E L W$ estimates of $d$ in all experiments. In the interests of space, we present results relating only to the $E L W$ estimate in Tables 1 to 10. The results relating to $L W$ are qualitatively similar and are reported in the accompanying on-line supplementary appendix. ${ }^{3}$

\subsection{Empirical Coverage Rates for $S_{T}^{\mu}$}

First, we consider the $A R F I M A(0, d, 0)$ case where $\phi=\theta=0$. Here, we observe from the results in the top panel of Table 1 that the application of various bootstraps on levels data, including the sieve AR bootstrap which is valid for long memory series, does not in general yield empirical coverage rates close to the nominal 0.90 level. For $d=0$ and $T=120$ using $E L W$ we see that $S D D B_{M A}$ has the highest coverage rate, among the bootstrap methods considered, of 0.784 , while Sieve $_{H}$ has the lowest coverage rate of 0.599 . The performance of each of the bootstrap methods deteriorates further for $d>0$. The KN modification to the block bootstraps can be seen to yield improvements to empirical coverage rates. Looking at the middle panel of Table 1 , we see that the $b_{5}$ block choice returns coverage rates for both MBB and SBB in the range of $0.832-0.874$ in the smaller sample size $T=120$, with further improvements for the larger sample size, $T=500$.

The results in the bottom panel of Table 1 relate to our proposed FDB methods. Improvements in empirical coverage rates are seen using the FDB method for all of the bootstrap methods considered across all DGPs. For $T=120$, we observe that the best coverage rates for the $M B B$ using the $\mathrm{FDB}$, taken across the range of values of $d$ considered, is $M B B_{b_{1}}^{F D B}$

\footnotetext{
${ }^{3}$ It is easily verified that the statistics reported in our simulations satisfy the regularity conditions imposed by Assumption 3; see the discussion in Remark 15. With the exception of $L W$ for $d=0.75$, these statistics also all have continuous limiting distributions as required by Theorem 1 across the DGPs we consider; see the discussion in Remark 18. We have nonetheless chosen to retain $L W$ for $d=0.75$ in the full set of results reported in the supplementary appendix for reasons of comparison for the interested reader.
} 
which delivers coverage rates in the range of $0.823-0.870$. Compared to the best performing $M B B$ applied to the levels data, $M B B_{b^{*}}$, we see that there is therefore a very significant improvement in the lower bound of the range of coverage rates from 0.460 to 0.860 displayed by the latter across $d$. The best performing $S B B$ using the FDB is $S B B_{b_{1}}^{F D B}$ which displays empirical coverage rates in the range of 0.805-0.859. Comparing it to the best $S B B$ method applied directly to the levels data, $S B B_{b^{*}}$, we see that this constitutes an increase of 0.345 in the lower bound seen for the range of coverage rates of the latter which is $0.458-0.783$. Moreover, the FDB method also improves the coverage rates of both $S D D B_{M A}$ and $S D D B_{A R}$. The range of empirical coverage rates when $S D D B_{M A}$ and $S D D B_{A R}$ are applied directly to levels data is $0.473-0.783$ and $0.527-0.784$, respectively, across $d$. The corresponding FDB versions provide improved coverage rates in the range of $0.827-0.869$ and $0.825-0.872$ for $S D D B_{M A}^{F D B}$ and $S D D B_{A R}^{F D B}$, respectively. The FDB method also improves on the coverage rate of the sieve bootstrap methods. For example, the empirical coverage rates for Sieve $A I C$ and Sieve $e_{A I C}^{F D B}$, taken across all of the reported cases for $T=120$, lie in the range 0.397-0.682 and $0.644-0.847$, respectively.

Similar improvements are also seen to be delivered by the FDB method for the larger sample size, $T=500$. In particular, the best performing $M B B, M B B_{b^{*}}$, has coverage rates in the range of $0.384-0.800$ and the best $S B B, S B B_{b^{*}}$, has coverage rates in the range of 0.389-0.800. The corresponding FDB variants, $M B B_{b^{*}}^{F D B}$ and $S B B_{b^{*}}^{F D B}$, have empirical coverage rates in the range of $0.825-0.876$ and $0.819-0.875$, respectively. Comparing $M B B_{b^{*}}$ and $S B B_{b^{*}}$ to the best performing FDB variants, which are $M B B_{b_{1}}^{F D B}$ and $S B B_{b_{1}}^{F D B}$, we see that there are further improvements in performance, with both FDBs yielding coverage rates in the range $0.832-0.877$ across $d$. $S D D B_{M A}^{F D B}$ and $S D D B_{A R}^{F D B}$ also improve on the standard $S D D B_{M A}$ and $S D D B_{A R}$ increasing the empirical coverage rates from the range of $0.466-0.803$ and $0.515-0.799$, respectively, to $0.833-0.885$ and $0.836-0.885$, respectively. The Sieve $_{A I C}$ bootstrap which has coverage rates in the range of $0.415-0.782$ is also improved by the FDB. In particular, Sieve ${ }_{A I C}^{F D B}$ offers coverage rates in the range of $0.813-0.844$.

Consider next the results for the persistent $A R F I M A(1, d, 0)$ case where $\phi=0.8$ reported in Table 2. In relation to the results in Table 1 , empirical coverage rates are significantly decreased for all approaches with none delivering close to the nominal 0.90 level. This is not unexpected as $u_{t}$ is approaching the degree of persistence shown by a unit root process which creates two sources of difficulty: (i) the semiparametric estimation of $d$ deteriorates, which will affect all of the bootstrap methods, and (ii) the underlying (approximately) short memory process displays a strong pattern of persistence (recall that, other than the sieve, the bootstrap resampling methods are valid for weak dependence alone). The KN modification seems now to work only for $M B B_{b_{6}}^{K N}$ with $d=0.25$ and $d=0.35$ and for the larger sample size considered. These results are also not particularly robust; we see that when $d$ is small, there is a downward bias in the empirical coverage rates, while for larger values of $d$ the coverage rates display an upward bias. Applying the FDB certainly improves on the 
standard bootstrap methods, but still fails to deliver empirical coverage rates close to the nominal level. For example, for $T=500$, the best performing $M B B$ is $M B B_{b^{*}}$ with coverage rates in the range of $0.173-0.438$ across all $d$, while the corresponding $\mathrm{FDB}, M B B_{b^{*}}^{F D B}$, has improved coverage rates in the range of $0.564-0.590$. Similarly, $S B B_{b^{*}}$ is improved using $S B B_{b^{*}}^{F D B}$ with coverage rates improving from 0.186-0.436 to 0.550-0.586. $S D D B_{M A}^{F D B}$ and $S D D B_{A R}^{F D B}$ also improve on the standard $S D D B_{M A}$ and $S D D B_{A R}$ methods with empirical coverage rates improving from $0.219-0.464$ and $0.363-0.516$, respectively, to $0.595-0.631$

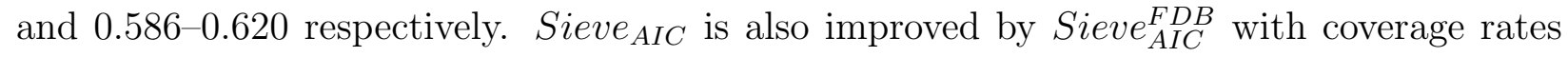
increasing from the range $0.261-0.443$ to $0.607-0.645$ across all $d$.

Next, consider the ARFIMA(1,d,0) case with $\phi=-0.8$. The results in Table 3 show that there are KN bootstrap variants which yield satisfactory results across all choices of $d$, even for $T=120$. However, it is important to note that, for this DGP, the choice of the block length results in bootstraps with an upward bias in their coverage rates, as seen for example with $M B B_{b_{6}}^{K N}$ for $d \geq 0.45$. The FDB variants also provide accurate coverage rates across most cases. As before, we can still find block bootstrap variants, e.g., $M B B_{b_{1}}^{F D B}$, which display an upward bias as with the $\mathrm{KN}$ variants. However it is important to note the impact of the block length. In particular, we see that $M B B_{b_{1}}^{F D B}$ has coverage rates in the range of 0.969-0.987, but $M B B_{b^{*}}^{F D B}$ shows coverage rates closer to the nominal level in the range of $0.858-0.884$, across all $d$. For $T=500$, the best performing $M B B$, across all $d$, is $M B B_{b^{*}}$ when directly applied to the original levels data with coverage rates in the range of $0.417-$ 0.842. The best performing FDB variant is $M B B_{b_{3}}^{F D B}$ which has improved coverage rates in the range of $0.902-0.94$ across all $d$. $S D D B_{M A}^{F D B}$ and $S D D B_{A R}^{F D B}$ improve the standard $S D D B_{M A}$ and $S D D B_{A R}$ increasing their coverage rates from $0.452-0.822$ and $0.544-0.818$, respectively, to $0.805-0.865$ and $0.805-0.863$, respectively. Sieve $A I C$ is also improved by Sieve $_{A I C}^{F D B}$ with coverage rates increasing from the range of $0.446-0.787$ to $0.839-0.864$ across all $d$.

Consider next the $\operatorname{ARFIMA}(0, d, 1)$ case. For $\theta=0.8$, the results in Table 4 show that the KN block bootstraps using $b_{5}$ and $b_{6}$ yield accurate coverage rates across all DGPs for both sample sizes. For FDB variants, we see that the larger sample size generally improves the coverage rates, which now are in the range of 0.764-0.888 across all $d$. For example, for $T=500, S D D B_{M A}, S D D B_{A R}$ and Sieve $_{A I C}$ have coverage rates in the range of $0.495-0.804$, $0.554-0.808$ and $0.510-0.776$, respectively. FDB improves the coverage rates of these to the ranges $0.812-0.888,0.820-0.886$ and $0.810-0.843$, respectively. For $\theta=-0.8$, the results in Table 5 highlight a tendency to upward bias across all the bootstrap methods considered. Moreover, for $T=500$ almost all of the block bootstrap methods exceed the nominal 0.90 coverage rate. This is the case even for $d=0$, indicating that the block bootstraps do not accurately capture the dynamics of the series. $S D D B_{M A}^{F D B}$ and $S D D B_{A R}^{F D B}$ improve on the coverage rates of the standard $S D D B_{M A}$ and $S D D B_{A R}$ increasing these from $0.665-$ 0.906 and $0.675-0.938$, respectively, to $0.900-0.927$ and $0.899-0.930$, respectively. Sieve $A I C$ 
is also improved by $\operatorname{Sieve}_{A I C}^{F D B}$ with coverage rates improving from the range $0.741-0.960$ to 0.895-0.967 across all $d$.

\subsection{Coverage Rates for $S_{T}^{d}$}

Consider first the results for the $\operatorname{ARFIMA}(0, d, 0)$ case reported in Table 6 . Here we see, as expected, that the direct application of bootstraps to the levels data does not yield accurate coverage rates. This holds across all bootstrap variants for $T=120$, including the sieve AR bootstrap which, it must be recalled, is asymptotically valid for stationary long memory series. To illustrate, $M B B_{b^{*}}$ has coverage rates in the range $0.571-0.655$ and $S B B_{b^{*}}$ in the range $0.591-0.655$ across all $d$. The FDB is seen to improve these with $M B B_{b^{*}}^{F D B}$ and $S B B_{b^{*}}^{F D B}$ having empirical coverage rates in the ranges $0.806-0.875$ and 0.816-0.874, respectively. Moreover, the best FDB variants of the $M B B$ and $S B B$ methods are $M B B_{b_{1}}^{F D B}$ and $S B B_{b_{1}}^{F D B}$ which display coverage rates in the range 0.829-0.894 and $0.815-0.884$, respectively. $S D D B_{M A}, S D D B_{A R}$ and Sieve $_{A I C}$ have coverage rates in the ranges $0.581-0.863,0.577-0.772$ and $0.528-0.613$, respectively. The corresponding FDB

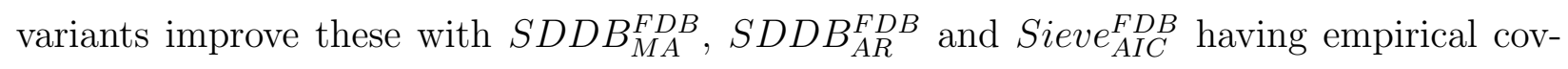
erage rates in the ranges $0.819-0.886,0.819-0.887$ and $0.714-0.786$, respectively. For the larger sample, $T=500$, we see that $S D D B_{M A}$ and $S D D B_{A R}$ return coverage rates closer to the nominal value for $d \in\{0.35,0.45,0.49,0.75\}$. Also, Sieve ${ }_{H}$ has good coverage rates for $d \in\{0.15,0.25,0.35,0.45,0.49\}$. Moreover, the FDB approach improves the coverage rates of $S D D B_{M A}, S D D B_{A R}$ and Sieve $_{H}$ from the range 0.526-0.892, 0.523-0.861, 0.731-0.894 to $0.839-0.902,0.837-0.904$ and $0.797-0.839$, respectively.

Table 7 reports the coverage rates for the persistent $A R F I M A(1, d, 0)$ case with $\phi=0.8$. Here we see that none of the bootstrap variants applied directly to the original series returns coverage rates close to the nominal 0.90 value. In particular, the largest coverage rate we have across both sample sizes and all methods is 0.736 . FDB improves most bootstraps, most evidently so for the larger sample size. For $T=500, M B B_{b_{3}}$ and $S B B_{b_{3}}$ have very poor empirical coverage rates in the range of $0.003-0.361$ and $0.012-0.389$, respectively, across all $d$. The FDB method very significantly improves these rates with $M B B_{b_{3}}^{F D B}$ and $S B B_{b_{3}}^{F D B}$ having coverage rates in the range $0.652-0.720$ and $0.635-0.717$, respectively. Moreover, $S D D B_{M A}, S D D B_{A R}$ and Sieve $_{A I C}$ have empirical coverage rates in the range $0.455-0.642$, 0.427-0.648 and 0.435-0.572, respectively, across all $d$, with the FDB improving these to $0.635-0.725,0.653-0.723$ and $0.598-0.678$, respectively.

Tables 8 and 9 present the results for $A R F I M A(1, d, 0)$ with $\phi=-0.8$ and $A R F I M A(0, d, 1)$, with $\theta=0.8$, respectively. In both tables we see that bootstrapping the original series directly yields unsatisfactory results for most methods. Particularly in Table 8 , we see that for ARFIMA $(1, d, 0)$ with $\phi=-0.8$, only Sieve $_{H}$ for $T=500$ has coverage rates in the range of $0.765-0.851$, which are relatively close to the nominal 0.90 value, particularly for $d \geq 0.35$. 
For $A R F I M A(0, d, 1)$, with $\theta=0.8$, in Table 9 , we see that Sieve $_{H}$ provides coverage rates in the range $0.504-0.904$ using the larger sample.

The FDB approach improves coverage rates for all of the bootstraps. Using the FDB, we see in Table 8 that for $T=120$, the best coverage rates for $M B B$ are given by $M B B_{b_{2}}^{F D B}$ and are in the range $0.846-0.906$ and for $S B B$ are given by $S B B_{b_{2}}^{F D B}$ and are in the range 0.858-0.898. For $T=500$ the best $M B B$ and $S B B$ are $M B B_{b_{2}}^{F D B}$ and $S B B_{b_{1}}^{F D B}$ with coverage rates in the range $0.881-0.900$ and $0.862-0.888$, respectively, across $d$.

Similarly, in Table 9 we see that for $T=120, M B B_{b_{2}}^{F D}$ has coverage rates in the range of 0.801-0.899. In the larger sample, these rates change to 0.850-0.904. We also see that the $F D B$ improves the other bootstrap variants as well. In particular, we see that in the

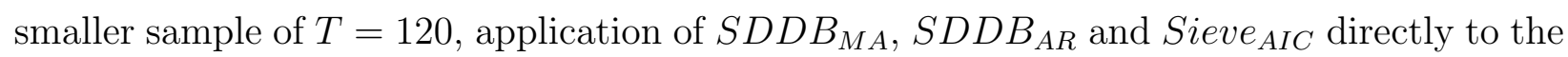
original levels data yield coverage rates in the range 0.595-0.836, 0.594-0.810, 0.604-0.707, respectively, across all $d$. FDB improves these coverage rates to $0.775-0.861,0.772-0.867$ and 0.673-0.756 respectively. In the larger sample, $S D D B_{M A}, S D D B_{A R}$ and Sieve $_{A I C}$ have coverage rates in the range of $0.559-0.886,0.495-0.836$ and $0.678-0.860$, respectively. FDB improves these rates to $0.831-0.877,0.830-0.876$ and $0.779-0.812$, respectively.

Finally, Table 10 presents the results for $\operatorname{ARFIMA}(0, d, 1)$, with $\theta=-0.8$. As for the results in Table 7 , we again see that most of the bootstrap methods do not return accurate coverage rates when they are applied directly to the original levels data. The FDB method is seen to improve the empirical coverage rates of all bootstraps by more than $30 \%$ (on average across all DGPs). For $T=500$, we note from Table 10 that the application of $M B B_{b^{*}}, S B B_{b^{*}}, S D D B_{M A}$ and Sieve $A I C$ directly to original levels data delivers coverage rates in the range $0.503-0.789,0.518-0.810,0.524-0.739$ and $0.387-0.599$, respectively, across all $d . M B B_{b^{*}}^{F D B}, S B B_{b^{*}}^{F D B}, S D D B_{M A}^{F D B}$ and Sieve ${ }_{A I C}^{F D B}$ improve the coverage rate range to $0.701-0.743,0.685-0.735,0.688-0.714,0.686-0.721$ and $0.659-0.729$, respectively.

We conclude this section by providing some recommendations based on our simulation results. As discussed above, we deliberately included in our study a number of DGPs known to be extremely challenging from the perspective of obtaining reliable inference in finite samples. Our simulation results indicate that the FDB method yields significant improvements in empirical bootstrap coverage rates (compared with the corresponding standard bootstrap methods applied to the levels of the data) in these cases while also performing very well for less challenging DGPs. Overall, we would recommend the FDB method paired with the $S D D B$ (MA or AR) bootstrap, SINCE this appears to deliver the most reliable performance among the methods considered. 


\section{Conclusions}

In this paper we have discussed a fractional differencing-based bootstrap methodology. The idea underlying this approach is simple, intuitive and applicable to both stationary and nonstationary fractionally integrated series. In the first step one estimates the fractional integration parameter using any consistent estimation method. One then obtains an approximately short memory series by applying the fractional differencing operator based on this estimate to the data. One then implements any valid block or residual-based resampling method on this fractionally differenced series. One next applies the fractional cumulating operator, based again on the estimate for the fractional integration parameter from the original data, to form the fractionally integrated bootstrap counterpart data. The material in this paper extends the original work on the fractional differencing bootstrap in Kapetanios and Papailias (2011) and shares some similarities with Poskitt, Grose and Martin (2015), who investigate the case of the sieve bootstrap for stationary fractionally integrated processes.

Our contribution to the literature has been to provide a more general framework which can be applied to both stationary and nonstationary fractionally integrated processes, and to show that this can be used with any valid bootstrap resampling method. The finite sample performance of the fractional differencing bootstrap was demonstrated in a series of simulation experiments. Two statistics were considered: the first based on the unconditional mean of the fractionally integrated process, and the second based on the fractional exponent. The simulation evidence suggested that the proposed methodology can offer significant improvements in empirical bootstrap coverage rates for both of these statistics to the corresponding standard bootstrap methods applied to the levels of the data.

\section{References}

Abadir, M.K., Distaso, W., Giraitis, L., 2007. Nonstationarity-extended local Whittle estimation. Journal of Econometrics, 141, 1353-1384.

Andrews, D.WK., Sun, Y., 2004. Adaptive local polynomial Whittle estimation of long-range dependence. Econometrica, 72, 569-614.

Bickel, P.J., Freedman, D.A., 1981. Some asymptotic theory for the bootstrap. Annals of Statistics, 9, 1196-1217.

Bühlmann, P., 1997. Sieve bootstrap for time series. Bernoulli, 3, 123-148.

Bühlmann, P., Künsch, H.R., 1999. Block length selection in the bootstrap for time series. Computational Statistics and Data Analysis, 31, 295-310.

Carlstein, E., 1986. The use of subseries values for estimating the variance of a general statistic from a stationary sequence. Annals of Statistics, 14, 1171-1179. 
Cavaliere, G., Nielsen, M.Ø., Taylor, A.M.R., 2017. Quasi-maximum likelihood estimation and bootstrap inference in fractional time series models with heteroskedasticity of unknown form. Journal of Econometrics, 198, 165-188.

Efron, B., 1979. Bootstrap methods: another look at the jackknife. Annals of Statistics, 7, $1-26$.

Fox, C., Taqqu, M.S., 1986. Large sample properties of parameter estimates for strongly dependent processes. Annals of Statistics, 14, 517-532.

Fragkeskou, M., Paparoditis, E., 2018. Extending the range of validity of the autoregressive (sieve) bootstrap. Journal of Time Series Analysis, 39, 356-379.

Frederiksen, P., Nielsen, M.Ø., 2008. Bias-reduced estimation of long-memory stochastic volatility. Journal of Financial Econometrics, 6, 496-512.

Frederiksen, P., Nielsen, F.S., Nielsen, M.Ø, 2012. Local polynomial Whittle estimation of perturbed fractional processes. Journal of Econometrics, 167, 426-447.

Geweke, J., Porter-Hudak, S., 1983. The estimation and application of long memory time series models. Journal of Time Series Analysis, 4, 221-238.

Haldrup, N., Nielsen, M.Ø., 2007. Estimation of fractional integration in the presence of data noise. Computational Statistics \& Data Analysis, 51, 3100-3114.

Hall, P., Horowitz, J.L., Jing, B.Y., 1995. On blocking rules for the bootstrap with dependent data. Biometrika, 82, 561-574.

Hosking, J.R.M., 1996. Asymptotic distributions of the sample mean, autocovariance, and autocorrelations of long-memory time series. Journal of Econometrics, 73, 261-284.

Hosoya, Y., 1997. A limit theory for long range dependence and statistical inference on related models. Annals of Statistics, 25, 105-137.

Hualde, J., Robinson, P.M., 2011. Gaussian pseudo-maximum likelihood estimation of fractional time Series. Annals of Statistics, 39, 3152-3181.

Jensen, A.N., Nielsen, M.Ø., 2014. A fast fractional difference algorithm. Journal of Time Series Analysis, 35, 428-436.

Johansen, S., Nielsen, M.Ø., 2010. Likelihood inference for a nonstationary fractional autoregressive model. Journal of Econometrics, 158, 51-66.

Johansen, S., Nielsen, M.Ø., 2012. A necessary moment condition for the fractional functional central limit theorem. Econometric Theory, 28, 671-679. 
Johansen, S., Nielsen, M.Ø., 2016. The role of initial values in conditional sum-of-squares estimation of nonstationary fractional time series models. Econometric Theory, 32, 10951139.

Kapetanios, G., 2010. A generalization of a sieve bootstrap invariance principle to long memory processes. Quantitative and Qualitative Analysis in Social Sciences, 4-1, 19-40. Also available as: "Kapetanios, G., 2004. A bootstrap invariance principle for highly nonstationary long memory processes. School of Economics and Finance, Queen Mary University of London, Working Paper No. 507.".

Kapetanios, G., Papailias, F., 2011. Block bootstrap and long memory. School of Economics and Finance, Queen Mary University of London, Working Paper No. 679.

Kapetanios, G., Psaradakis, Z., 2006. Sieve bootstrap for strongly dependent stationary processes. School of Economics and Finance, Queen Mary University of London, Working Paper No. 552.

Kim, Y.M.K., Nordman, D.J., 2011. Properties of a block bootstrap under long-range dependence. Sankhya: The Indian Journal of Statistics, 73-A, Part 1, 79-109.

Krampe, J., Kreiss, J.-P., Paparoditis, E., 2018. Estimated Wold representation and spectraldensity-driven bootstrap for time series. Journal of the Royal Statistical Society: Series B, Forthcoming.

Kreiss, J.-P., 1992. Bootstrap Procedures for $A R(\infty)$ Processes. Springer: Heidelberg.

Kreiss, J.-P., Paparoditis, E., Politis, D.N., 2011. On the range of validity of the autoregressive sieve bootstrap. Annals of Statistics, 39, 2103-2130.

Künsch, H.R., 1989. The jackknife and the bootstrap for general stationary observations. Annals of Statistics, 7, 1-26.

Lahiri, S.N., 1993. On the moving block bootstrap under long range dependence. Statistics and Probability Letters, 18, 405-413.

Nielsen, M.Ø., 2005. Semiparametric estimation in time-series regression with long-range dependence. Journal of Time Series Analysis, 26, 279-304.

Nielsen, M.Ø., 2015. Asymptotics for the conditional-sum-of-squares estimator in multivariate fractional timeseries models. Journal of Time Series Analysis, 36, 154-188.

Nielsen, M.Ø., Frederiksen, P.H., 2005. Finite sample comparison of parametric, semiparametric, and wavelet estimators of fractional integration. Econometric Reviews, 24, 405-443. 
Papailias, F., Dias, G.F., 2015. Forecasting long memory series subject to structural change: A two-stage approach. International Journal of Forecasting, 31, 1056-1066.

Patton, A., Politis, D.N., White, H., 2009. Correction to "Automatic block-length selection for the dependent bootstrap". Econometric Reviews, 23, 53-70.

Politis, D.N., Romano, J.P., 1992. A circular block resampling procedure for stationary data. In Exploring the Limits of Bootstrap, (LePage, R., Billard, L., eds.), 263-270. Wiley: New York.

Politis, D.N., Romano, J.P., 1994. The stationary bootstrap. Journal of American Statistical Association, 89, 1303-1313.

Politis, D.N., White, H., 2004. Automatic block-length selection for the dependent bootstrap. Econometric Reviews, 23, 53-70.

Poskitt, D.S., 2008. Properties of the sieve bootstrap for fractionally integrated and noninvertible processes. Journal of Time Series Analysis, 29, 224-250.

Poskitt, D.S., Grose, S.D., Martin, G.M., 2015. Higher-order improvements of the sieve bootstrap for fractionally integrated processes. Journal of Econometrics, 188, 94-110.

Robinson, P.M., 1995. Gaussian semi-parametric estimation of long range dependence. Annals of Statistics, 23, 1630-1661.

Robinson, P. M., 2006. Conditional-sum-of-squares estimation of models for stationary time series with long memory. JIMS Lecture Notes-Monograph Series: Time Series and Related Topics, 52, 130-137.

Shimotsu, K., 2010. Exact local Whittle estimation of fractional integration with unknown mean and time trend. Econometric Theory, 26, 501-540.

Shimotsu, K., Phillips, P.C.B., 2005. Exact local Whittle estimation of fractional integration. Annals of Statistics, 33, 1890-1933.

Shimotsu, K., Phillips, P.C.B., 2006. Local Whittle estimation and some of its variants. Journal of Econometrics, 130, 209-233.

Sowell, F.B., 1992. Maximum likelihood estimation of stationary univariate fractionally integrated time series models. Journal of Econometrics, 53, 165-188. 


\section{Tables}

\begin{tabular}{|c|c|c|c|c|c|c|c|c|c|c|c|c|}
\hline \multicolumn{13}{|c|}{$A R F I M A(0, d, 0), S_{T}^{\mu}$} \\
\hline \multirow{3}{*}{ Bootstrap \d } & \multicolumn{12}{|c|}{$E L W$} \\
\hline & \multicolumn{6}{|c|}{$T=120$} & \multicolumn{6}{|c|}{$T=500$} \\
\hline & 0 & 0.15 & 0.25 & 0.35 & 0.45 & 0.49 & 0 & 0.15 & 0.25 & 0.35 & 0.45 & 0.49 \\
\hline$M B B_{b_{1}}$ & 0.779 & 0.676 & 0.555 & 0.482 & 0.377 & 0.361 & 0.801 & 0.609 & 0.468 & 0.331 & 0.255 & 0.191 \\
\hline$M B B_{b_{2}}$ & 0.778 & 0.673 & 0.552 & 0.482 & 0.370 & 0.366 & 0.804 & 0.635 & 0.508 & 0.360 & 0.291 & 0.235 \\
\hline$M B B_{b_{3}}$ & 0.779 & 0.696 & 0.589 & 0.519 & 0.413 & 0.414 & 0.804 & 0.658 & 0.535 & 0.396 & 0.325 & 0.269 \\
\hline$M B B_{b^{*}}$ & 0.781 & 0.676 & 0.571 & 0.533 & 0.460 & 0.466 & 0.800 & 0.643 & 0.568 & 0.479 & 0.448 & 0.389 \\
\hline$S B B_{b_{1}}$ & 0.776 & 0.685 & 0.562 & 0.490 & 0.390 & 0.391 & 0.803 & 0.625 & 0.489 & 0.353 & 0.276 & 0.221 \\
\hline$S B B_{b_{2}}$ & 0.783 & 0.684 & 0.567 & 0.501 & 0.398 & 0.390 & 0.806 & 0.646 & 0.523 & 0.390 & 0.324 & 0.276 \\
\hline$S B B_{b_{3}}$ & 0.782 & 0.696 & 0.590 & 0.523 & 0.428 & 0.426 & 0.807 & 0.667 & 0.547 & 0.431 & 0.371 & 0.308 \\
\hline$S B B_{b^{*}}$ & 0.783 & 0.671 & 0.579 & 0.537 & 0.460 & 0.458 & 0.802 & 0.645 & 0.586 & 0.490 & 0.451 & 0.399 \\
\hline$S D D B_{M A}$ & 0.783 & 0.659 & 0.568 & 0.527 & 0.473 & 0.481 & 0.803 & 0.620 & 0.566 & 0.512 & 0.501 & 0.466 \\
\hline$S D D B_{A R}$ & 0.784 & 0.650 & 0.569 & 0.552 & 0.527 & 0.544 & 0.799 & 0.617 & 0.567 & 0.515 & 0.547 & 0.547 \\
\hline Sieve $_{A I C}$ & 0.682 & 0.588 & 0.520 & 0.462 & 0.397 & 0.414 & 0.782 & 0.637 & 0.567 & 0.474 & 0.451 & 0.415 \\
\hline Sieve $_{H}$ & 0.599 & 0.548 & 0.502 & 0.457 & 0.428 & 0.416 & 0.756 & 0.679 & 0.624 & 0.556 & 0.552 & 0.557 \\
\hline$M B B_{b_{4}}^{K \Lambda}$ & 0.821 & 0.785 & 0.731 & 0.706 & 0.649 & 0.657 & 0.847 & 0.794 & 0.744 & 0.696 & 0.713 & 0.695 \\
\hline$M B B_{b_{5}}^{K_{4} N}$ & 0.842 & 0.853 & 0.832 & 0.867 & 0.855 & 0.874 & 0.849 & 0.860 & 0.863 & 0.875 & 0.921 & 0.918 \\
\hline$M B B_{b_{6}}^{b_{5} N}$ & 0.827 & 0.880 & 0.889 & 0.946 & 0.965 & 0.975 & 0.841 & 0.906 & 0.950 & 0.973 & 0.992 & 0.998 \\
\hline$S B B_{b_{4}}^{K_{6} N}$ & 0.823 & 0.786 & 0.733 & 0.707 & 0.673 & 0.675 & 0.836 & 0.804 & 0.772 & 0.739 & 0.759 & 0.757 \\
\hline$S B B_{b_{5}}^{K_{4} N}$ & 0.836 & 0.838 & 0.834 & 0.851 & 0.852 & 0.867 & 0.856 & 0.876 & 0.882 & 0.901 & 0.934 & 0.939 \\
\hline$S B B_{b_{6}}^{K N}$ & 0.809 & 0.854 & 0.883 & 0.929 & 0.947 & 0.960 & 0.841 & 0.912 & 0.944 & 0.970 & 0.990 & 0.997 \\
\hline$M B B_{b_{1}}^{F D B}$ & 0.870 & 0.870 & 0.860 & 0.860 & 0.826 & 0.823 & 0.877 & 0.877 & 0.855 & 0.843 & 0.857 & 0.832 \\
\hline$M B B_{b_{2}}^{O_{1}} D B$ & 0.864 & 0.868 & 0.851 & 0.850 & 0.821 & 0.824 & 0.859 & 0.878 & 0.853 & 0.841 & 0.841 & 0.832 \\
\hline$M B B_{b_{3}}^{b_{2} D B}$ & 0.853 & 0.858 & 0.839 & 0.849 & 0.812 & 0.816 & 0.867 & 0.872 & 0.848 & 0.820 & 0.828 & 0.824 \\
\hline$M B B_{b^{*}}^{b_{3} D B}$ & 0.856 & 0.859 & 0.847 & 0.844 & 0.801 & 0.817 & 0.868 & 0.876 & 0.847 & 0.834 & 0.840 & 0.825 \\
\hline$S B B_{b_{1}}^{F D B}$ & 0.861 & 0.863 & 0.841 & 0.847 & 0.817 & 0.816 & 0.861 & 0.874 & 0.859 & 0.845 & 0.846 & 0.832 \\
\hline$S B B_{b_{2}}^{b_{1} D B}$ & 0.853 & 0.862 & 0.855 & 0.851 & 0.811 & 0.818 & 0.865 & 0.875 & 0.850 & 0.825 & 0.835 & 0.823 \\
\hline$S B B_{b_{3}}^{b_{2} D B}$ & 0.843 & 0.850 & 0.837 & 0.840 & 0.797 & 0.794 & 0.857 & 0.865 & 0.837 & 0.818 & 0.824 & 0.815 \\
\hline$S B B_{b^{*}}^{F D B}$ & 0.859 & 0.855 & 0.841 & 0.834 & 0.797 & 0.805 & 0.867 & 0.875 & 0.844 & 0.827 & 0.831 & 0.819 \\
\hline$S D D B_{M A}^{F D}$ & 0.861 & 0.869 & 0.858 & 0.852 & 0.827 & 0.827 & 0.876 & 0.885 & 0.862 & 0.835 & 0.846 & 0.833 \\
\hline$S D D B_{A R}^{F D B}$ & 0.866 & 0.872 & 0.860 & 0.848 & 0.828 & 0.825 & 0.879 & 0.885 & 0.866 & 0.843 & 0.850 & 0.836 \\
\hline Sieve $_{A I B}^{F D B}$ & 0.644 & 0.733 & 0.771 & 0.818 & 0.817 & 0.847 & 0.813 & 0.843 & 0.830 & 0.821 & 0.844 & 0.831 \\
\hline Sieve ${ }_{H}^{F D B}$ & 0.505 & 0.602 & 0.647 & 0.686 & 0.730 & 0.769 & 0.751 & 0.792 & 0.775 & 0.767 & 0.793 & 0.778 \\
\hline
\end{tabular}

Table 1: Empirical Coverage Rates for $S_{T}^{\mu}$ using $A R F I M A(0, d, 0)$ DGPs.

Notes: The top panel of the table shows the coverage rates for the bootstrap methods outlined in Section 2 directly applied to the original levels series. The middle panel shows the coverage rates for block bootstraps applying the KN methodology. The bottom panel shows the coverage rates for the bootstrap methods using the suggested FDB methodology. 


\begin{tabular}{|c|c|c|c|c|c|c|c|c|c|c|c|c|}
\hline \multicolumn{13}{|c|}{$\operatorname{ARFIMA}(1, d, 0), \phi=0.8, S_{T}^{\mu}$} \\
\hline \multirow{3}{*}{ Bootstrap \d } & \multicolumn{12}{|c|}{$E L W$} \\
\hline & \multicolumn{6}{|c|}{$T=120$} & \multicolumn{6}{|c|}{$T=500$} \\
\hline & 0 & 0.15 & 0.25 & 0.35 & 0.45 & 0.49 & 0 & 0.15 & 0.25 & 0.35 & 0.45 & 0.49 \\
\hline$M B B_{b_{1}}$ & 0.134 & 0.082 & 0.076 & 0.058 & 0.074 & 0.058 & 0.312 & 0.200 & 0.152 & 0.110 & 0.094 & 0.076 \\
\hline$M B B_{b_{2}}$ & 0.138 & 0.083 & 0.077 & 0.059 & 0.073 & 0.059 & 0.351 & 0.235 & 0.189 & 0.142 & 0.106 & 0.097 \\
\hline$M B B_{b_{3}}$ & 0.162 & 0.096 & 0.090 & 0.069 & 0.091 & 0.071 & 0.390 & 0.267 & 0.218 & 0.165 & 0.127 & 0.117 \\
\hline$M B B_{b^{*}}$ & 0.170 & 0.110 & 0.107 & 0.090 & 0.109 & 0.093 & 0.438 & 0.340 & 0.270 & 0.227 & 0.189 & 0.173 \\
\hline$S B B_{b_{1}}$ & 0.151 & 0.087 & 0.083 & 0.066 & 0.088 & 0.066 & 0.336 & 0.226 & 0.184 & 0.136 & 0.110 & 0.092 \\
\hline$S B B_{b_{2}}$ & 0.152 & 0.086 & 0.087 & 0.065 & 0.086 & 0.066 & 0.380 & 0.258 & 0.209 & 0.158 & 0.122 & 0.115 \\
\hline$S B B_{b_{3}}$ & 0.162 & 0.103 & 0.095 & 0.073 & 0.102 & 0.076 & 0.401 & 0.283 & 0.230 & 0.182 & 0.146 & 0.128 \\
\hline$S B B_{b^{*}}$ & 0.170 & 0.109 & 0.106 & 0.088 & 0.106 & 0.096 & 0.436 & 0.336 & 0.277 & 0.225 & 0.196 & 0.186 \\
\hline$S D D B_{M A}$ & 0.191 & 0.123 & 0.122 & 0.105 & 0.124 & 0.117 & 0.464 & 0.366 & 0.308 & 0.265 & 0.234 & 0.219 \\
\hline$S D D B_{A R}$ & 0.225 & 0.273 & 0.365 & 0.513 & 0.628 & 0.663 & 0.467 & 0.374 & 0.363 & 0.383 & 0.459 & 0.516 \\
\hline Sieve $_{A I C}$ & 0.147 & 0.103 & 0.102 & 0.097 & 0.132 & 0.123 & 0.443 & 0.353 & 0.306 & 0.282 & 0.261 & 0.276 \\
\hline Sieve $_{H}$ & 0.130 & 0.101 & 0.119 & 0.141 & 0.184 & 0.176 & 0.408 & 0.362 & 0.408 & 0.452 & 0.527 & 0.588 \\
\hline$M B B_{b_{4}}^{K N}$ & 0.227 & 0.190 & 0.181 & 0.184 & 0.206 & 0.187 & 0.520 & 0.495 & 0.460 & 0.457 & 0.442 & 0.453 \\
\hline$M B B_{b_{5}}^{K N} N$ & 0.349 & 0.357 & 0.372 & 0.404 & 0.458 & 0.440 & 0.604 & 0.627 & 0.638 & 0.684 & 0.725 & 0.746 \\
\hline$M B B_{b_{6}}^{b_{5} N}$ & 0.444 & 0.506 & 0.576 & 0.663 & 0.775 & 0.781 & 0.662 & 0.763 & 0.821 & 0.914 & 0.953 & 0.959 \\
\hline$S B B_{b_{4}}^{K_{6} N}$ & 0.245 & 0.200 & 0.193 & 0.203 & 0.237 & 0.210 & 0.535 & 0.516 & 0.493 & 0.498 & 0.515 & 0.514 \\
\hline$S B B_{b_{5}}^{K} N$ & 0.331 & 0.321 & 0.366 & 0.404 & 0.464 & 0.435 & 0.592 & 0.650 & 0.650 & 0.714 & 0.758 & 0.780 \\
\hline$S B B_{b_{6}}^{K N}$ & 0.419 & 0.451 & 0.531 & 0.591 & 0.716 & 0.719 & 0.635 & 0.742 & 0.811 & 0.900 & 0.935 & 0.949 \\
\hline$M B B_{b_{1}}^{F D B}$ & 0.306 & 0.283 & 0.283 & 0.292 & 0.308 & 0.285 & 0.452 & 0.470 & 0.456 & 0.451 & 0.431 & 0.448 \\
\hline$M B B_{b_{2}}^{O_{1} D B}$ & 0.308 & 0.278 & 0.282 & 0.293 & 0.310 & 0.283 & 0.524 & 0.525 & 0.505 & 0.511 & 0.501 & 0.500 \\
\hline$M B B_{b_{3}}^{b_{2} D B}$ & 0.323 & 0.303 & 0.307 & 0.317 & 0.334 & 0.316 & 0.567 & 0.573 & 0.545 & 0.562 & 0.544 & 0.551 \\
\hline$M B B_{b^{*}}^{D_{3}} D B$ & 0.319 & 0.293 & 0.311 & 0.305 & 0.337 & 0.308 & 0.590 & 0.587 & 0.568 & 0.575 & 0.564 & 0.579 \\
\hline$S B B_{b_{1}}^{F D B}$ & 0.309 & 0.285 & 0.284 & 0.293 & 0.316 & 0.296 & 0.498 & 0.505 & 0.486 & 0.497 & 0.473 & 0.477 \\
\hline$S B B_{b_{2}}^{F D B}$ & 0.312 & 0.286 & 0.278 & 0.301 & 0.310 & 0.297 & 0.538 & 0.550 & 0.527 & 0.534 & 0.522 & 0.534 \\
\hline$S B B_{b_{3}}^{F_{2} D B}$ & 0.321 & 0.294 & 0.285 & 0.309 & 0.326 & 0.308 & 0.575 & 0.575 & 0.550 & 0.561 & 0.546 & 0.549 \\
\hline$S B B_{b^{*}}^{D_{3}} D B$ & 0.317 & 0.291 & 0.286 & 0.304 & 0.325 & 0.301 & 0.574 & 0.586 & 0.564 & 0.571 & 0.550 & 0.560 \\
\hline$S D D B_{M A}^{F D B}$ & 0.334 & 0.311 & 0.306 & 0.324 & 0.331 & 0.326 & 0.631 & 0.620 & 0.595 & 0.607 & 0.591 & 0.598 \\
\hline$S D D B_{A R}^{F D B}$ & 0.346 & 0.317 & 0.303 & 0.331 & 0.338 & 0.326 & 0.620 & 0.615 & 0.597 & 0.612 & 0.586 & 0.606 \\
\hline Sieve $_{A I C}^{F D B}$ & 0.317 & 0.322 & 0.346 & 0.371 & 0.424 & 0.411 & 0.612 & 0.623 & 0.607 & 0.645 & 0.624 & 0.635 \\
\hline Sieve $_{H}^{F D B}$ & 0.229 & 0.221 & 0.250 & 0.281 & 0.324 & 0.322 & 0.516 & 0.540 & 0.529 & 0.547 & 0.540 & 0.558 \\
\hline
\end{tabular}

Table 2: Empirical Coverage Rates for $S_{T}^{\mu}$ using $A R F I M A(1, d, 0), \phi=0.8$ DGPs.

Notes: The top panel of the table shows the coverage rates for the bootstrap methods outlined in Section 2 directly applied to the original levels series. The middle panel shows the coverage rates for block bootstraps applying the KN methodology. The bottom panel shows the coverage rates for the bootstrap methods using the suggested FDB methodology. 


\begin{tabular}{|c|c|c|c|c|c|c|c|c|c|c|c|c|}
\hline \multicolumn{13}{|c|}{$\operatorname{ARFIMA}(1, d, 0), \phi=-0.8, S_{T}^{\mu}$} \\
\hline \multirow{3}{*}{ Bootstrap \d } & \multicolumn{12}{|c|}{$E L W$} \\
\hline & \multicolumn{6}{|c|}{$T=120$} & \multicolumn{6}{|c|}{$T=500$} \\
\hline & 0 & 0.15 & 0.25 & 0.35 & 0.45 & 0.49 & 0 & 0.15 & 0.25 & 0.35 & 0.45 & 0.49 \\
\hline$M B B_{b_{1}}$ & 0.929 & 0.803 & 0.716 & 0.580 & 0.478 & 0.437 & 0.935 & 0.738 & 0.581 & 0.414 & 0.295 & 0.258 \\
\hline$M B B_{b_{2}}$ & 0.930 & 0.801 & 0.716 & 0.582 & 0.476 & 0.439 & 0.896 & 0.710 & 0.572 & 0.417 & 0.309 & 0.276 \\
\hline$M B B_{b_{3}}$ & 0.891 & 0.768 & 0.704 & 0.583 & 0.494 & 0.458 & 0.858 & 0.675 & 0.561 & 0.443 & 0.333 & 0.313 \\
\hline$M B B_{b^{*}}$ & 0.840 & 0.746 & 0.696 & 0.576 & 0.523 & 0.494 & 0.842 & 0.696 & 0.601 & 0.504 & 0.442 & 0.417 \\
\hline$S B B_{b_{1}}$ & 0.922 & 0.789 & 0.719 & 0.587 & 0.491 & 0.453 & 0.928 & 0.728 & 0.587 & 0.420 & 0.313 & 0.278 \\
\hline$S B B_{b_{2}}$ & 0.924 & 0.790 & 0.710 & 0.578 & 0.489 & 0.448 & 0.892 & 0.706 & 0.581 & 0.438 & 0.339 & 0.309 \\
\hline$S B B_{b_{3}}$ & 0.897 & 0.771 & 0.702 & 0.588 & 0.512 & 0.471 & 0.878 & 0.699 & 0.594 & 0.472 & 0.371 & 0.347 \\
\hline$S B B_{b^{*}}$ & 0.859 & 0.767 & 0.711 & 0.598 & 0.535 & 0.507 & 0.858 & 0.720 & 0.616 & 0.522 & 0.459 & 0.430 \\
\hline$S D D B_{M A}$ & 0.827 & 0.741 & 0.692 & 0.599 & 0.554 & 0.511 & 0.822 & 0.702 & 0.620 & 0.513 & 0.452 & 0.454 \\
\hline$S D D B_{A R}$ & 0.837 & 0.761 & 0.714 & 0.631 & 0.606 & 0.599 & 0.818 & 0.699 & 0.617 & 0.544 & 0.611 & 0.621 \\
\hline Sieve $_{A I C}$ & 0.753 & 0.627 & 0.596 & 0.492 & 0.462 & 0.444 & 0.787 & 0.650 & 0.602 & 0.494 & 0.457 & 0.446 \\
\hline Sieve $_{H}$ & 0.729 & 0.621 & 0.575 & 0.498 & 0.451 & 0.426 & 0.789 & 0.692 & 0.636 & 0.574 & 0.532 & 0.536 \\
\hline$M B B_{b_{4}}^{K N}$ & 0.935 & 0.853 & 0.837 & 0.758 & 0.736 & 0.688 & 0.895 & 0.835 & 0.791 & 0.765 & 0.721 & 0.730 \\
\hline$M B B_{b_{5}}^{b_{4} N}$ & 0.910 & 0.890 & 0.885 & 0.884 & 0.900 & 0.898 & 0.888 & 0.886 & 0.888 & 0.901 & 0.918 & 0.928 \\
\hline$M B B_{b_{6}}^{b_{5}} N$ & 0.868 & 0.891 & 0.925 & 0.949 & 0.973 & 0.972 & 0.872 & 0.930 & 0.956 & 0.976 & 0.993 & 0.998 \\
\hline$S B B_{b_{4}}^{b_{6}}$ & 0.939 & 0.855 & 0.840 & 0.766 & 0.760 & 0.722 & 0.910 & 0.852 & 0.809 & 0.789 & 0.759 & 0.791 \\
\hline$S B B_{b_{5}}^{b_{4}} N$ & 0.920 & 0.880 & 0.891 & 0.881 & 0.897 & 0.897 & 0.895 & 0.904 & 0.892 & 0.921 & 0.933 & 0.943 \\
\hline$S B B_{b_{6}}^{K N}$ & 0.885 & 0.900 & 0.928 & 0.943 & 0.967 & 0.968 & 0.877 & 0.931 & 0.948 & 0.986 & 0.988 & 0.993 \\
\hline$M B B_{b_{1}}^{F D B}$ & 0.969 & 0.978 & 0.987 & 0.977 & 0.984 & 0.985 & 0.991 & 0.995 & 0.983 & 0.992 & 0.991 & 0.983 \\
\hline$M B B_{b_{2}}^{b_{1}} D B$ & 0.966 & 0.981 & 0.987 & 0.982 & 0.983 & 0.981 & 0.978 & 0.972 & 0.966 & 0.976 & 0.960 & 0.955 \\
\hline$M B B_{b_{3}}^{b_{2} D B}$ & 0.948 & 0.962 & 0.966 & 0.955 & 0.961 & 0.948 & 0.932 & 0.940 & 0.914 & 0.927 & 0.914 & 0.902 \\
\hline$M B B_{b^{*}}^{F} D B$ & 0.860 & 0.865 & 0.884 & 0.870 & 0.868 & 0.858 & 0.877 & 0.894 & 0.862 & 0.862 & 0.848 & 0.848 \\
\hline$S B B_{b_{1}}^{F D B}$ & 0.964 & 0.977 & 0.984 & 0.974 & 0.977 & 0.978 & 0.988 & 0.987 & 0.976 & 0.986 & 0.978 & 0.976 \\
\hline$S B B_{h_{2}}^{O_{1}} D B$ & 0.969 & 0.977 & 0.979 & 0.974 & 0.984 & 0.980 & 0.970 & 0.965 & 0.954 & 0.967 & 0.953 & 0.951 \\
\hline$S B B_{b_{3}}^{b_{2} D B}$ & 0.951 & 0.957 & 0.965 & 0.955 & 0.959 & 0.954 & 0.951 & 0.952 & 0.932 & 0.937 & 0.923 & 0.918 \\
\hline$S B B_{b^{*}}^{b_{3}} D B$ & 0.894 & 0.876 & 0.893 & 0.874 & 0.887 & 0.878 & 0.883 & 0.895 & 0.862 & 0.861 & 0.846 & 0.849 \\
\hline$S D D^{\circ} B^{F D B}$ & 0.845 & 0.817 & 0.852 & 0.817 & 0.839 & 0.812 & 0.855 & 0.865 & 0.836 & 0.834 & 0.805 & 0.819 \\
\hline$S D D B_{A R}^{F D B}$ & 0.863 & 0.841 & 0.872 & 0.831 & 0.852 & 0.827 & 0.863 & 0.858 & 0.840 & 0.825 & 0.805 & 0.809 \\
\hline Sieve $_{A I C}^{A D B}$ & 0.659 & 0.755 & 0.813 & 0.849 & 0.907 & 0.912 & 0.839 & 0.864 & 0.864 & 0.856 & 0.853 & 0.849 \\
\hline Sieve $_{H}^{F D B}$ & 0.604 & 0.737 & 0.798 & 0.822 & 0.893 & 0.900 & 0.793 & 0.837 & 0.835 & 0.836 & 0.826 & 0.844 \\
\hline
\end{tabular}

Table 3: Empirical Coverage Rates for $S_{T}^{\mu}$ using $A R F I M A(1, d, 0), \phi=-0.8$ DGPs.

Notes: The top panel of the table shows the coverage rates for the bootstrap methods outlined in Section 2 directly applied to the original levels series. The middle panel shows the coverage rates for block bootstraps applying the KN methodology. The bottom panel shows the coverage rates for the bootstrap methods using the suggested FDB methodology. 


\begin{tabular}{|c|c|c|c|c|c|c|c|c|c|c|c|c|}
\hline \multicolumn{13}{|c|}{$\operatorname{ARFIMA}(0, d, 1), \theta=0.8, S_{T}^{\mu}$} \\
\hline \multirow{3}{*}{ Bootstrap \d } & \multicolumn{12}{|c|}{$E L W$} \\
\hline & \multicolumn{6}{|c|}{$T=120$} & \multicolumn{6}{|c|}{$T=500$} \\
\hline & 0 & 0.15 & 0.25 & 0.35 & 0.45 & 0.49 & 0 & 0.15 & 0.25 & 0.35 & 0.45 & 0.49 \\
\hline$M B B_{b_{1}}$ & 0.717 & 0.615 & 0.481 & 0.430 & 0.370 & 0.336 & 0.776 & 0.594 & 0.479 & 0.320 & 0.261 & 0.231 \\
\hline$M B B_{b_{2}}$ & 0.724 & 0.621 & 0.486 & 0.433 & 0.366 & 0.334 & 0.787 & 0.629 & 0.515 & 0.362 & 0.295 & 0.269 \\
\hline$M B B_{b_{3}}$ & 0.729 & 0.639 & 0.514 & 0.461 & 0.406 & 0.384 & 0.788 & 0.644 & 0.556 & 0.391 & 0.328 & 0.307 \\
\hline$M B B_{b^{*}}$ & 0.713 & 0.642 & 0.530 & 0.489 & 0.449 & 0.433 & 0.786 & 0.662 & 0.603 & 0.488 & 0.449 & 0.423 \\
\hline$S B B_{b_{1}}$ & 0.712 & 0.619 & 0.489 & 0.439 & 0.387 & 0.358 & 0.777 & 0.609 & 0.497 & 0.338 & 0.278 & 0.251 \\
\hline$S B B_{b_{2}}$ & 0.718 & 0.621 & 0.491 & 0.446 & 0.388 & 0.360 & 0.790 & 0.632 & 0.546 & 0.387 & 0.321 & 0.306 \\
\hline$S B B_{b_{3}}$ & 0.715 & 0.641 & 0.513 & 0.471 & 0.416 & 0.399 & 0.795 & 0.659 & 0.575 & 0.427 & 0.361 & 0.340 \\
\hline$S B B_{b^{*}}$ & 0.721 & 0.643 & 0.526 & 0.490 & 0.444 & 0.437 & 0.786 & 0.672 & 0.611 & 0.488 & 0.453 & 0.432 \\
\hline$S D D B_{M A}$ & 0.736 & 0.649 & 0.541 & 0.516 & 0.476 & 0.476 & 0.804 & 0.660 & 0.626 & 0.538 & 0.513 & 0.495 \\
\hline$S D D B_{A R}$ & 0.736 & 0.650 & 0.557 & 0.540 & 0.522 & 0.534 & 0.808 & 0.666 & 0.627 & 0.554 & 0.579 & 0.596 \\
\hline Sieve $_{A I C}$ & 0.618 & 0.550 & 0.479 & 0.461 & 0.445 & 0.432 & 0.766 & 0.677 & 0.625 & 0.528 & 0.519 & 0.510 \\
\hline Sieve $_{H}$ & 0.577 & 0.546 & 0.441 & 0.439 & 0.463 & 0.453 & 0.761 & 0.685 & 0.652 & 0.613 & 0.636 & 0.667 \\
\hline$M B B_{b_{4}}^{K}$ & 0.771 & 0.741 & 0.691 & 0.649 & 0.630 & 0.630 & 0.837 & 0.818 & 0.777 & 0.731 & 0.729 & 0.723 \\
\hline$M B B_{b_{5}}^{K N} N$ & 0.800 & 0.832 & 0.816 & 0.837 & 0.869 & 0.860 & 0.848 & 0.882 & 0.875 & 0.891 & 0.924 & 0.922 \\
\hline$M B B_{b_{6}}^{K} N$ & 0.795 & 0.875 & 0.897 & 0.929 & 0.966 & 0.956 & 0.850 & 0.923 & 0.947 & 0.975 & 0.996 & 0.995 \\
\hline$S B B_{b_{4}}^{K_{6} N}$ & 0.765 & 0.740 & 0.688 & 0.669 & 0.644 & 0.652 & 0.844 & 0.825 & 0.794 & 0.764 & 0.777 & 0.772 \\
\hline$S B B_{b_{5}}^{K} N$ & 0.799 & 0.811 & 0.806 & 0.815 & 0.866 & 0.859 & 0.855 & 0.886 & 0.885 & 0.914 & 0.934 & 0.936 \\
\hline$S B B_{b_{6}}^{K N}$ & 0.772 & 0.850 & 0.861 & 0.908 & 0.946 & 0.954 & 0.842 & 0.922 & 0.938 & 0.977 & 0.993 & 0.996 \\
\hline$M B B_{b_{1}}^{F D B}$ & 0.804 & 0.829 & 0.799 & 0.798 & 0.784 & 0.775 & 0.848 & 0.859 & 0.853 & 0.817 & 0.813 & 0.795 \\
\hline$M B B_{b_{2}}^{O_{1} D B}$ & 0.806 & 0.818 & 0.801 & 0.795 & 0.799 & 0.773 & 0.859 & 0.876 & 0.846 & 0.825 & 0.820 & 0.806 \\
\hline$M B B_{b_{3}}^{D_{2} D B}$ & 0.798 & 0.821 & 0.801 & 0.797 & 0.792 & 0.776 & 0.862 & 0.872 & 0.850 & 0.828 & 0.829 & 0.808 \\
\hline$M B B_{b^{*}}^{D_{3}} D B$ & 0.791 & 0.822 & 0.793 & 0.790 & 0.778 & 0.770 & 0.849 & 0.870 & 0.849 & 0.831 & 0.823 & 0.799 \\
\hline$S B B_{b_{1}}^{F D B}$ & 0.793 & 0.815 & 0.784 & 0.787 & 0.775 & 0.767 & 0.839 & 0.864 & 0.845 & 0.815 & 0.815 & 0.797 \\
\hline$S B B_{b_{2}}^{F D}$ & 0.797 & 0.800 & 0.789 & 0.783 & 0.779 & 0.764 & 0.860 & 0.874 & 0.851 & 0.823 & 0.827 & 0.807 \\
\hline$S B B_{b_{3}}^{F_{2} D B}$ & 0.790 & 0.817 & 0.781 & 0.778 & 0.780 & 0.768 & 0.858 & 0.869 & 0.847 & 0.819 & 0.819 & 0.797 \\
\hline$S B B_{b^{*}}^{F} D B$ & 0.779 & 0.806 & 0.770 & 0.770 & 0.756 & 0.755 & 0.853 & 0.869 & 0.847 & 0.813 & 0.820 & 0.793 \\
\hline$S D D B_{M A}^{F D B}$ & 0.823 & 0.838 & 0.798 & 0.794 & 0.800 & 0.784 & 0.870 & 0.888 & 0.873 & 0.840 & 0.835 & 0.812 \\
\hline$S D D B_{A R}^{F D B}$ & 0.829 & 0.839 & 0.811 & 0.800 & 0.799 & 0.788 & 0.868 & 0.886 & 0.868 & 0.846 & 0.845 & 0.820 \\
\hline Sieve $_{A I C}^{F D B}$ & 0.568 & 0.663 & 0.681 & 0.750 & 0.777 & 0.806 & 0.806 & 0.843 & 0.839 & 0.812 & 0.814 & 0.810 \\
\hline Sieve $_{H}^{F D B}$ & 0.509 & 0.594 & 0.606 & 0.666 & 0.723 & 0.742 & 0.764 & 0.789 & 0.804 & 0.792 & 0.794 & 0.771 \\
\hline
\end{tabular}

Table 4: Empirical Coverage Rates for $S_{T}^{\mu}$ using $A R F I M A(0, d, 1), \theta=0.8$ DGPs.

Notes: The top panel of the table shows the coverage rates for the bootstrap methods outlined in Section 2 directly applied to the original levels series. The middle panel shows the coverage rates for block bootstraps applying the KN methodology. The bottom panel shows the coverage rates for the bootstrap methods using the suggested FDB methodology. 


\begin{tabular}{|c|c|c|c|c|c|c|c|c|c|c|c|c|}
\hline \multicolumn{13}{|c|}{$\operatorname{ARFIMA}(0, d, 1), \theta=-0.8, S_{T}^{\mu}$} \\
\hline \multirow{3}{*}{ Bootstrap \d } & \multicolumn{12}{|c|}{$E L W$} \\
\hline & \multicolumn{6}{|c|}{$T=120$} & \multicolumn{6}{|c|}{$T=500$} \\
\hline & 0 & 0.15 & 0.25 & 0.35 & 0.45 & 0.49 & 0 & 0.15 & 0.25 & 0.35 & 0.45 & 0.49 \\
\hline$M B B_{b_{1}}$ & 0.999 & 0.996 & 0.986 & 0.985 & 0.963 & 0.947 & 0.999 & 0.997 & 0.956 & 0.889 & 0.749 & 0.715 \\
\hline$M B B_{b_{2}}$ & 0.999 & 0.997 & 0.986 & 0.985 & 0.960 & 0.947 & 0.999 & 0.990 & 0.935 & 0.859 & 0.731 & 0.694 \\
\hline$M B B_{b_{3}}$ & 0.998 & 0.991 & 0.984 & 0.978 & 0.956 & 0.943 & 0.991 & 0.984 & 0.917 & 0.851 & 0.725 & 0.706 \\
\hline$M B B_{b^{*}}$ & 0.979 & 0.972 & 0.966 & 0.964 & 0.958 & 0.948 & 0.966 & 0.955 & 0.894 & 0.848 & 0.732 & 0.708 \\
\hline$S B B_{b_{1}}$ & 0.998 & 0.997 & 0.986 & 0.985 & 0.963 & 0.952 & 0.999 & 0.996 & 0.955 & 0.892 & 0.751 & 0.722 \\
\hline$S B B_{b_{2}}$ & 0.999 & 0.997 & 0.988 & 0.986 & 0.959 & 0.954 & 0.999 & 0.992 & 0.936 & 0.873 & 0.742 & 0.724 \\
\hline$S B B_{b_{3}}$ & 0.998 & 0.995 & 0.984 & 0.979 & 0.958 & 0.946 & 0.992 & 0.986 & 0.931 & 0.863 & 0.750 & 0.731 \\
\hline$S B B_{b^{*}}$ & 0.995 & 0.987 & 0.973 & 0.974 & 0.961 & 0.949 & 0.977 & 0.967 & 0.904 & 0.876 & 0.761 & 0.734 \\
\hline$S D D B_{M A}$ & 0.987 & 0.972 & 0.963 & 0.962 & 0.951 & 0.936 & 0.906 & 0.939 & 0.891 & 0.828 & 0.700 & 0.665 \\
\hline$S D D B_{A R}$ & 0.986 & 0.971 & 0.960 & 0.962 & 0.949 & 0.936 & 0.902 & 0.938 & 0.888 & 0.826 & 0.703 & 0.675 \\
\hline Sieve $_{A I C}$ & 0.986 & 0.968 & 0.951 & 0.933 & 0.911 & 0.909 & 0.960 & 0.929 & 0.858 & 0.810 & 0.741 & 0.747 \\
\hline Sieve $_{H}$ & 0.985 & 0.964 & 0.950 & 0.928 & 0.910 & 0.919 & 0.957 & 0.945 & 0.877 & 0.882 & 0.836 & 0.837 \\
\hline$M B B_{b_{4}}^{K N}$ & 0.999 & 0.997 & 0.995 & 0.995 & 0.989 & 0.984 & 0.998 & 0.995 & 0.978 & 0.962 & 0.943 & 0.941 \\
\hline$M B B_{b_{5}}^{K N} N$ & 0.996 & 0.991 & 0.997 & 0.998 & 0.995 & 0.994 & 0.989 & 0.995 & 0.987 & 0.984 & 0.986 & 0.985 \\
\hline$M B B_{b_{6}}^{b_{5} N}$ & 0.972 & 0.971 & 0.983 & 0.985 & 0.991 & 0.995 & 0.971 & 0.994 & 0.988 & 0.998 & 0.999 & 0.999 \\
\hline$S B B_{b_{4}}^{K_{6} N}$ & 0.999 & 0.997 & 0.997 & 0.997 & 0.992 & 0.989 & 0.997 & 0.996 & 0.981 & 0.979 & 0.961 & 0.957 \\
\hline$S B B_{b_{5}}^{K} N$ & 0.999 & 0.997 & 0.998 & 0.998 & 0.997 & 0.996 & 0.995 & 0.996 & 0.990 & 0.995 & 0.991 & 0.992 \\
\hline$S B B_{b_{6}}^{K N}$ & 0.999 & 0.997 & 0.999 & 1.000 & 1.000 & 0.999 & 0.990 & 0.996 & 0.994 & 0.997 & 1.000 & 1.000 \\
\hline$M B B_{b_{1}}^{F D B}$ & 0.933 & 0.964 & 0.958 & 0.976 & 0.981 & 0.983 & 0.992 & 1.000 & 0.999 & 1.000 & 1.000 & 1.000 \\
\hline$M B B_{b_{2}}^{O_{1} D B}$ & 0.933 & 0.956 & 0.953 & 0.974 & 0.983 & 0.984 & 0.991 & 0.999 & 0.998 & 1.000 & 1.000 & 1.000 \\
\hline$M B B_{b_{3}}^{D_{2} D B}$ & 0.914 & 0.960 & 0.947 & 0.981 & 0.984 & 0.985 & 0.987 & 0.997 & 0.999 & 1.000 & 0.999 & 1.000 \\
\hline$M B B_{b^{*}}^{D_{3}} D B$ & 0.885 & 0.932 & 0.934 & 0.961 & 0.979 & 0.975 & 0.951 & 0.988 & 0.971 & 0.978 & 0.970 & 0.963 \\
\hline$S B B_{b_{1}}^{F D B}$ & 0.945 & 0.964 & 0.962 & 0.978 & 0.982 & 0.985 & 0.995 & 1.000 & 0.998 & 1.000 & 1.000 & 1.000 \\
\hline$S B B_{b_{2}}^{F D B}$ & 0.948 & 0.964 & 0.960 & 0.978 & 0.982 & 0.987 & 0.995 & 1.000 & 0.999 & 1.000 & 1.000 & 1.000 \\
\hline$S B B_{b_{3}}^{F_{2} D B}$ & 0.941 & 0.963 & 0.958 & 0.980 & 0.985 & 0.987 & 0.991 & 0.999 & 0.999 & 1.000 & 0.999 & 1.000 \\
\hline$S B B_{b^{*}}^{D_{3}} D B$ & 0.930 & 0.958 & 0.954 & 0.983 & 0.984 & 0.984 & 0.967 & 0.994 & 0.978 & 0.982 & 0.968 & 0.969 \\
\hline$S D D B_{M A}^{F D B}$ & 0.902 & 0.939 & 0.930 & 0.952 & 0.965 & 0.941 & 0.911 & 0.927 & 0.920 & 0.907 & 0.909 & 0.900 \\
\hline$S D D B_{A R}^{F D B}$ & 0.906 & 0.949 & 0.937 & 0.950 & 0.967 & 0.943 & 0.916 & 0.930 & 0.917 & 0.908 & 0.909 & 0.899 \\
\hline Sieve $_{A I C}^{F D B}$ & 0.589 & 0.693 & 0.746 & 0.834 & 0.876 & 0.905 & 0.895 & 0.963 & 0.955 & 0.967 & 0.958 & 0.963 \\
\hline Sieve $_{H}^{F D B}$ & 0.570 & 0.659 & 0.713 & 0.802 & 0.855 & 0.882 & 0.897 & 0.959 & 0.947 & 0.964 & 0.949 & 0.959 \\
\hline
\end{tabular}

Table 5: Empirical Coverage Rates for $S_{T}^{\mu}$ using $A R F I M A(0, d, 1), \theta=-0.8$ DGPs.

Notes: The top panel of the table shows the coverage rates for the bootstrap methods outlined in Section 2 directly applied to the original levels series. The middle panel shows the coverage rates for block bootstraps applying the KN methodology. The bottom panel shows the coverage rates for the bootstrap methods using the suggested FDB methodology. 


\begin{tabular}{|c|c|c|c|c|c|c|c|c|c|c|c|c|c|c|}
\hline \multicolumn{15}{|c|}{$\operatorname{ARFIMA}(0, d, 0), S_{T}^{d}$} \\
\hline \multirow{3}{*}{ Bootstrap \d } & \multicolumn{14}{|c|}{$E L W$} \\
\hline & \multicolumn{7}{|c|}{$T=120$} & \multicolumn{7}{|c|}{$T=500$} \\
\hline & 0 & 0.15 & 0.25 & 0.35 & 0.45 & 0.49 & 0.75 & 0 & 0.15 & 0.25 & 0.35 & 0.45 & 0.49 & 0.75 \\
\hline$M B B_{b_{1}}$ & 0.612 & 0.576 & 0.532 & .466 & 0.381 & 0.376 & 0.167 & 0.579 & 0.511 & 0.412 & 0.314 & 0.193 & 0.134 & .031 \\
\hline$M B B_{b_{2}}$ & 0.613 & 0.588 & 0.543 & 0.468 & 0.390 & 0.377 & 0.167 & 0.580 & 0.518 & 0.420 & 0.328 & 0.209 & 0.145 & 0.030 \\
\hline$M B B_{b_{3}}$ & 0.629 & 0.602 & 0.561 & 0.498 & 0.435 & 0.423 & 0.217 & 0.581 & 0.537 & 0.435 & 0.345 & 0.236 & 58 & 042 \\
\hline$M B B_{b^{*}}$ & 0.612 & 0.581 & 0.571 & 0.591 & 0.624 & 0.655 & 0.635 & 0.586 & 0.539 & 0.551 & 0.660 & 0.684 & 0.671 & 0.614 \\
\hline$S B B_{b_{1}}$ & 0.633 & 0.606 & 0.568 & 0.516 & 0.444 & 0.431 & 0.227 & 0.579 & 0.525 & 0.430 & 0.333 & 0.218 & 0.159 & 0.040 \\
\hline$S B I$ & 0.630 & 0.611 & 0.568 & 0.511 & 0.448 & 0.437 & 0.230 & 0.606 & 0.551 & 0.471 & 0.389 & 0.273 & 7 & .069 \\
\hline$S B B_{b_{3}}$ & 0.654 & 0.646 & 0.619 & 0.574 & 0.531 & 0.516 & 0.345 & 0.618 & 0.588 & 0.520 & 0.445 & 0.361 & 8 & 0.122 \\
\hline$S B B_{b^{*}}$ & 24 & 0.591 & 0.611 & 0.624 & 0.649 & 0.655 & 0.623 & 0.589 & & 0.626 & & 99 & & 618 \\
\hline$S D D B$ & 610 & 0.587 & 0.581 & 0.638 & 0.759 & 0.817 & 0.863 & 0.578 & 0.526 & 0.553 & 0.809 & 0.892 & 0.871 & 0.811 \\
\hline$S D D B_{A R}$ & .620 & 0.586 & 0.577 & 0.624 & 0.722 & 0.772 & 0.719 & 0.585 & 0.523 & 43 & 0.815 & 0.861 & & .551 \\
\hline Siev & 534 & 0.531 & 0.528 & 0.533 & 0.543 & 0.565 & 0.613 & 0.583 & & 0.582 & 0.658 & 89 & & 0.756 \\
\hline Sieve $_{H}$ & 0.654 & 0.676 & 0.662 & 0.697 & 0.692 & 0.692 & 0.668 & 0.774 & 0.818 & 0.846 & 0.877 & 0.894 & 0.885 & 0.731 \\
\hline$M B B_{b_{1}}^{F L}$ & 0.877 & 0.886 & 0.883 & 0.878 & 0.886 & 0.894 & 0.829 & 0.876 & 0.866 & 0.896 & 0.900 & 0.875 & 0.883 & 0.844 \\
\hline$M B B_{b_{2}}^{F D}$ & 0.868 & 0.892 & 0.883 & 0.872 & 0.878 & 0.889 & 0.826 & 0.858 & 0.862 & 0.890 & 0.900 & 0.872 & 0.885 & 0.847 \\
\hline$M B B_{b_{3}}^{F+D B}$ & 0.843 & 0.858 & 0.851 & 0.844 & 0.859 & 0.872 & 0.804 & 0.840 & 0.839 & 0.873 & 0.877 & 0.851 & 0.868 & 0.836 \\
\hline$M B B_{b^{*}}^{F} D B$ & 0.850 & 0.875 & 0.866 & 0.856 & 0.861 & 0.869 & 0.806 & 0.840 & 0.839 & 0.854 & 0.885 & 0.860 & 0.873 & 0.827 \\
\hline$S B B_{b_{1}}^{F D B}$ & 0.863 & 0.871 & 0.871 & 0.871 & 0.874 & 0.884 & 0.815 & 0.869 & 0.850 & 0.883 & 0.896 & 0.865 & 0.877 & 0.837 \\
\hline$S B B_{b_{2}}^{F} D B$ & 0.868 & 0.883 & 0.871 & 0.864 & 0.875 & 0.883 & 0.821 & 0.855 & 0.835 & 0.863 & 0.885 & 0.852 & 0.875 & 0.823 \\
\hline$S E$ & 0.857 & 0.858 & 0.854 & 0.853 & 0.862 & 0.867 & 0.808 & 0.836 & 0.834 & 0.857 & 0.876 & 0.849 & 0.867 & 0.824 \\
\hline$S B B_{b^{*}}^{F D B}$ & 0.853 & 0.874 & 0.864 & 0.858 & 0.859 & 0.873 & 0.816 & 0.838 & 0.833 & 0.866 & 0.871 & 0.859 & 0.870 & 0.835 \\
\hline$S D D B_{M A}^{F D B}$ & 0.876 & 0.886 & 0.884 & 0.870 & 0.874 & 0.876 & 0.819 & 0.871 & 0.853 & 0.880 & 0.902 & 0.876 & 0.890 & 0.839 \\
\hline$S D D B_{A R}^{F D B}$ & 0.870 & 0.887 & 0.879 & 0.863 & 0.872 & 0.875 & 0.819 & 0.870 & 0.858 & 0.890 & 0.904 & 0.878 & 0.889 & 0.837 \\
\hline Sieve $_{A I C}^{F D B}$ & 0.739 & 0.760 & 0.780 & 0.764 & 0.776 & 0.786 & 0.714 & 0.804 & 0.807 & 0.831 & 0.854 & 0.828 & 0.851 & 0.809 \\
\hline Sieve $_{H}^{F D B}$ & 0.678 & 0.686 & 0.690 & 0.702 & 0.702 & 0.700 & 0.641 & 0.799 & 0.824 & 0.834 & 0.839 & 0.832 & 0.815 & 0.797 \\
\hline
\end{tabular}

Table 6: Empirical Coverage Rates for $S_{T}^{d}$ using $A R F I M A(0, d, 0)$ DGPs.

Notes: The top panel of the table shows the coverage rates for the bootstrap methods outlined in Section 2 directly applied to the original levels series. The bottom panel shows

the coverage rates for the bootstrap methods using the suggested FDB methodology. 


\begin{tabular}{|c|c|c|c|c|c|c|c|c|c|c|c|c|c|c|}
\hline \multicolumn{15}{|c|}{$\operatorname{ARFIMA}(1, d, 0), \phi=0.8, S_{T}^{d}$} \\
\hline \multirow{3}{*}{ Bootstrap \d } & \multicolumn{14}{|c|}{$E L W$} \\
\hline & \multicolumn{7}{|c|}{$T=120$} & \multicolumn{7}{|c|}{$T=500$} \\
\hline & 0 & 0.15 & 0.25 & 0.35 & 0.45 & 0.49 & 0.75 & 0 & 0.15 & 0.25 & 0.35 & 0.45 & 0.49 & 0.75 \\
\hline$M B B_{b_{1}}$ & 0.172 & 0.093 & 0.069 & 0.048 & 0.032 & 0.034 & 0.008 & 0.315 & 0.187 & 0.114 & 0.067 & 0.024 & 0.014 & .001 \\
\hline$M B B_{b_{2}}$ & 0.162 & 0.096 & 0.069 & 0.048 & 0.037 & 0.037 & 0.008 & 0.328 & 0.195 & 0.119 & 0.071 & 0.028 & 0.016 & 0.001 \\
\hline$M B B_{b_{3}}$ & 0.192 & 0.116 & 0.087 & 0.060 & 0.047 & 0.046 & 0.012 & 0.361 & 0.234 & 0.138 & 0.081 & 0.037 & 0.029 & 0.003 \\
\hline$M B B_{b^{*}}$ & 0.215 & 0.162 & 0.146 & 0.134 & 0.136 & 0.136 & 0.142 & 0.419 & 0.370 & 0.334 & 0.316 & 0.295 & 0.287 & 0.182 \\
\hline$S B B_{b_{1}}$ & 0.180 & 0.113 & 0.084 & 0.067 & 0.051 & 0.048 & 0.014 & 0.331 & 0.213 & 0.127 & 0.081 & 0.033 & 0.024 & 0.002 \\
\hline$S B B_{b_{2}}$ & 0.187 & 0.110 & 0.087 & 0.063 & 0.052 & 0.045 & 0.012 & 0.367 & 0.249 & 0.158 & 0.093 & 0.055 & 0.039 & 0.005 \\
\hline$S B I$ & 0.202 & 0.133 & 0.101 & 0.080 & 0.074 & 0.068 & 0.024 & 0.389 & 0.292 & 0.193 & 0.131 & 0.086 & 0.066 & 0.012 \\
\hline$S B B_{b^{*}}$ & 0.213 & 0.167 & 0.148 & 0.138 & 0.146 & 0.132 & 0.113 & 0.428 & 0.392 & 0.338 & 0.320 & 0.299 & 0.300 & 0.190 \\
\hline$S D D B_{M}$ & 0.247 & 0.293 & 0.328 & 0.374 & 0.404 & 0.417 & 0.461 & 0.455 & 0.582 & 0.597 & 0.614 & 0.642 & 0.637 & 0.584 \\
\hline$S D D B_{A F}$ & 0.272 & 0.385 & 0.466 & 0.525 & 0.538 & 0.574 & 0.605 & 0.461 & 0.585 & 0.616 & 0.648 & 0.617 & 0.589 & 0.427 \\
\hline Siev & 0.189 & 0.154 & 0.137 & 0.160 & 0.166 & 0.152 & 0.157 & 0.448 & 0.474 & 0.492 & 0.521 & 0.572 & 0.570 & 0.435 \\
\hline & 0. & 0.214 & 0.264 & 0.267 & 0.304 & 0.287 & 0.251 & 0.482 & 0.608 & 0.666 & 0.647 & 0.632 & 0.601 & 0.348 \\
\hline$M B$ & 0.398 & 0.399 & 0.393 & 0.440 & 0.448 & 0.417 & 0.383 & 0.626 & 0.671 & 0.683 & 0.670 & 0.685 & 0.691 & 0.617 \\
\hline$M B B_{b D}^{F D}$ & 0.385 & 0.400 & 0.402 & 0.443 & 0.442 & 0.420 & 0.376 & 0.642 & 0.692 & 0.688 & 0.692 & 0.696 & 0.699 & 0.619 \\
\hline$M B B_{b_{3}}^{F} D B$ & 0.431 & 0.428 & 0.430 & 0.467 & 0.460 & 0.437 & 0.399 & 0.668 & 0.700 & 0.713 & 0.712 & 0.720 & 0.712 & 0.652 \\
\hline$M B B_{b^{*}}^{F^{3}} D B$ & 0.422 & 0.425 & 0.435 & 0.471 & 0.463 & 0.436 & 0.399 & 0.670 & 0.716 & 0.735 & 0.716 & 0.736 & 0.713 & 0.643 \\
\hline$S B B_{b}^{I}$ & 0.397 & 0.409 & 0.411 & 0.455 & 0.442 & 0.423 & 0.385 & 0.652 & 0.689 & 0.697 & 0.693 & 0.704 & 0.692 & 0.625 \\
\hline$S B B_{b_{2}}^{F} D B$ & 0.397 & 0.402 & 0.399 & 0.447 & 0.449 & 0.415 & 0.385 & 0.656 & 0.691 & 0.694 & 0.705 & 0.719 & 0.700 & 0.628 \\
\hline$S B B_{b_{2}}^{F_{2} D B}$ & 0.382 & 0.385 & 0.403 & 0.431 & 0.432 & 0.417 & 0.381 & 0.642 & 0.695 & 0.707 & 0.695 & 0.717 & 0.695 & 0.635 \\
\hline$S B B_{b^{*}}^{F^{*} D B}$ & 0.392 & 0.396 & 0.402 & 0.437 & 0.431 & 0.403 & 0.389 & 0.635 & 0.684 & 0.689 & 0.685 & 0.706 & 0.685 & 0.621 \\
\hline$S D D B_{M A}^{F D B}$ & 0.400 & 0.404 & 0.413 & 0.441 & 0.451 & 0.419 & 0.394 & 0.673 & 0.711 & 0.715 & 0.712 & 0.725 & 0.720 & 0.635 \\
\hline & 0.394 & 0.395 & 0.399 & 0.450 & 0.443 & 0.423 & 0.398 & 0.682 & 0.713 & 0.713 & 0.723 & 0.719 & 0.709 & 0.653 \\
\hline & 180 & 0.292 & 0.263 & 0.307 & 0.325 & 0.305 & 0.284 & 0.635 & 0.677 & 0.678 & 0.675 & 0.675 & 0.663 & 0.598 \\
\hline Siev & 0.190 & 0.170 & 0.162 & 0.202 & 0.211 & 0.220 & 0.224 & 0.488 & 0.508 & 0.521 & 0.530 & 0.534 & 0.506 & 0.514 \\
\hline
\end{tabular}

Table 7: Empirical Coverage Rates for $S_{T}^{d}$ using $A R F I M A(1, d, 0), \phi=0.8$ DGPs.

Notes: The top panel of the table shows the coverage rates for the bootstrap methods outlined in Section 2 directly applied to the original levels series. The bottom panel shows

the coverage rates for the bootstrap methods using the suggested FDB methodology. 


\begin{tabular}{|c|c|c|c|c|c|c|c|c|c|c|c|c|c|c|}
\hline \multicolumn{15}{|c|}{$\operatorname{ARFIMA}(1, d, 0), \phi=-0.8, S_{T}^{d}$} \\
\hline \multirow{3}{*}{ Bootstrap \d } & \multicolumn{14}{|c|}{$E L W$} \\
\hline & \multicolumn{7}{|c|}{$T=120$} & \multicolumn{7}{|c|}{$T=500$} \\
\hline & 0 & 0.15 & 0.25 & 0.35 & 0.45 & 0.49 & 0.75 & 0 & 0.15 & 0.25 & 0.35 & 0.45 & 0.49 & 0.75 \\
\hline$M B B_{b_{1}}$ & 0.626 & 0.629 & 0.583 & 0.518 & 0.452 & 0.402 & 0.210 & 0.600 & 0.529 & 0.404 & 0.344 & 0.217 & 0.187 & .030 \\
\hline$M B B_{b_{2}}$ & 0.624 & 0.622 & 0.579 & 0.518 & 0.449 & 0.411 & 0.209 & 0.602 & 0.523 & 0.403 & 0.356 & 0.213 & 0.188 & 0.037 \\
\hline$M B B_{b_{3}}$ & 0.622 & 0.635 & 0.600 & 0.550 & 0.482 & 0.454 & 0.264 & 0.606 & 0.525 & 0.412 & 0.364 & 0.242 & 0.207 & 0.048 \\
\hline$M B B_{b^{*}}$ & 0.704 & 0.648 & 0.620 & 0.615 & 0.621 & 0.622 & 0.670 & 0.667 & 0.566 & 0.532 & 0.597 & 0.666 & 0.662 & 0.638 \\
\hline$S B B_{b_{1}}$ & 0.639 & 0.628 & 0.591 & 0.523 & 0.482 & 0.437 & 0.271 & 0.605 & 0.517 & 0.399 & 0.356 & 0.230 & 0.193 & 0.040 \\
\hline$S B B_{b_{2}}$ & 0.637 & 0.626 & 0.586 & 0.525 & 0.473 & 0.445 & 0.287 & 0.602 & 0.537 & 0.426 & 0.396 & 0.286 & 0.246 & 0.066 \\
\hline$S B B_{b_{3}}$ & 0.659 & 0.639 & 0.633 & 0.587 & 0.551 & 0.523 & 0.408 & 0.621 & 0.565 & 0.477 & 0.460 & 0.380 & 0.333 & 0.122 \\
\hline$S B B_{b^{*}}$ & 0.695 & 0.664 & 0.654 & 0.642 & 0.649 & 0.632 & 0.655 & 0.676 & 0.617 & 0.594 & 0.649 & 0.698 & 0.663 & 0.658 \\
\hline$S D I$ & 0.724 & 0.734 & 0.716 & 0.727 & 0.755 & 0.749 & 0.806 & 0.672 & 0.657 & 0.593 & 0.653 & 0.738 & 0.781 & 0.853 \\
\hline$S D D B_{A F}$ & 0.677 & 0.689 & 0.664 & 0.683 & 0.680 & 0.677 & 0.531 & 0.658 & 0.651 & 0.590 & 0.649 & 0.671 & 0.698 & 0.514 \\
\hline Siev & 0.561 & 0.572 & 0.572 & 0.565 & 0.591 & 0.599 & 0.640 & 0.589 & 0.561 & 0.535 & 0.637 & 0.730 & 0.718 & .810 \\
\hline & 0.658 & 0.655 & 0.662 & 0.665 & 0.694 & 0.708 & 0.714 & 0.765 & 0.799 & 0.791 & 0.835 & 0.851 & 0.851 & 0.842 \\
\hline$M E$ & 0.912 & 0.904 & 0.882 & 0.904 & 0.897 & 0.900 & 0.842 & 0.882 & 0.904 & 0.884 & 0.907 & 0.901 & 0.891 & 0.873 \\
\hline$M B B_{b D}^{F D}$ & 0.905 & 0.909 & 0.890 & 0.906 & 0.900 & 0.902 & 0.846 & 0.882 & 0.898 & 0.886 & 0.900 & 0.895 & 0.881 & 0.884 \\
\hline$M B B_{b_{3}}^{F} D B$ & 0.890 & 0.892 & 0.864 & 0.897 & 0.892 & 0.886 & 0.826 & 0.830 & 0.855 & 0.826 & 0.856 & 0.858 & 0.842 & 0.851 \\
\hline$M B B_{b^{*}}^{F^{3}} D B$ & 0.840 & 0.817 & 0.824 & 0.832 & 0.846 & 0.854 & 0.811 & 0.818 & 0.838 & 0.823 & 0.843 & 0.854 & 0.825 & 0.849 \\
\hline$S B B_{t}^{t}$ & 0.887 & 0.881 & 0.870 & 0.893 & 0.891 & 0.894 & 0.848 & 0.862 & 0.888 & 0.874 & 0.897 & 0.885 & 0.877 & 0.878 \\
\hline$S B B$ & 0.889 & 0.885 & 0.868 & 0.895 & 0.898 & 0.898 & 0.858 & 0.864 & 0.873 & 0.873 & 0.875 & 0.886 & 0.856 & 0.873 \\
\hline$S B B_{b_{2}}^{F_{2} D B}$ & 0.875 & 0.875 & 0.859 & 0.887 & 0.887 & 0.871 & 0.827 & 0.835 & 0.875 & 0.855 & 0.875 & 0.873 & 0.858 & 0.857 \\
\hline$S B E$ & 0.850 & 0.835 & 0.830 & 0.841 & 0.844 & 0.842 & 0.814 & 0.827 & 0.836 & 0.832 & 0.852 & 0.838 & 0.826 & 0.846 \\
\hline$S D D B_{M A}^{F D B}$ & 0.803 & 0.789 & 0.787 & 0.793 & 0.794 & 0.811 & 0.754 & 0.785 & 0.792 & 0.786 & 0.820 & 0.812 & 0.772 & 0.828 \\
\hline$S D D B^{i}$ & 0.760 & 0.739 & 0.745 & 0.768 & 0.750 & 0.762 & 0.708 & 0.779 & 0.799 & 0.777 & 0.811 & 0.825 & 0.775 & 0.821 \\
\hline & 886 & 0.788 & 0.767 & 0.780 & 0.769 & 0.772 & 0.700 & 0.822 & 0.840 & 0.818 & 0.835 & 0.839 & 0.813 & 0.846 \\
\hline Sie & 0.757 & 0.750 & 0.731 & 0.750 & 0.750 & 0.742 & 0.675 & 0.862 & 0.854 & 0.831 & 0.844 & 0.840 & 0.813 & 0.814 \\
\hline
\end{tabular}

Table 8: Empirical Coverage Rates for $S_{T}^{d}$ using $A R F I M A(1, d, 0), \phi=-0.8$ DGPs.

Notes: The top panel of the table shows the coverage rates for the bootstrap methods outlined in Section 2 directly applied to the original levels series. The bottom panel shows

the coverage rates for the bootstrap methods using the suggested FDB methodology. 


\begin{tabular}{|c|c|c|c|c|c|c|c|c|c|c|c|c|c|c|}
\hline \multicolumn{15}{|c|}{$\operatorname{ARFIMA}(0, d, 1), \theta=0.8, S_{T}^{d}$} \\
\hline \multirow{3}{*}{ Bootstrap \d } & \multicolumn{14}{|c|}{$E L W$} \\
\hline & \multicolumn{7}{|c|}{$T=120$} & \multicolumn{7}{|c|}{$T=500$} \\
\hline & 0 & 0.15 & 0.25 & 0.35 & 0.45 & 0.49 & 0.75 & 0 & 0.15 & 0.25 & 0.35 & 0.45 & 0.49 & 0.75 \\
\hline$M B B_{b_{1}}$ & 0.583 & 0.540 & 489 & 452 & 0.379 & 0.329 & 0.152 & 0.565 & 0.545 & 0.420 & 0.282 & 0.180 & 0.158 & .022 \\
\hline$M B B_{b_{2}}$ & 0.574 & 0.547 & 0.491 & 0.453 & 0.379 & 0.327 & 0.148 & 0.569 & 0.544 & 0.422 & 0.288 & 0.198 & 0.171 & 0.027 \\
\hline$M B B_{b_{3}}$ & 0.595 & 0.564 & 0.525 & 0.499 & 0.431 & 0.377 & 0.203 & 0.582 & 0.554 & 0.450 & 0.318 & 0.212 & 9 & 038 \\
\hline$M B B_{b^{*}}$ & 594 & 0.583 & 0.590 & 0.637 & 0.641 & 0.629 & 0.577 & 0.563 & 0.591 & 0.609 & 0.645 & 0.694 & 0.681 & 0.573 \\
\hline$S B B_{b_{1}}$ & 0.605 & 0.575 & 0.530 & 0.506 & 0.441 & 0.399 & 0.220 & 0.570 & 0.553 & 0.444 & 0.294 & 0.205 & 0.181 & 0.033 \\
\hline$S B B_{b_{2}}$ & 605 & 0.579 & 0.523 & 0.500 & 0.435 & 0.402 & 0.215 & 0.578 & 0.589 & 0.479 & 0.345 & 0.261 & & .053 \\
\hline$S B B_{b_{3}}$ & 0.635 & 0.622 & 0.579 & 0.581 & 0.509 & 0.480 & 0.318 & 0.602 & 0.607 & 0.524 & 0.421 & 0.344 & 7 & 0.108 \\
\hline$S B$ & 10 & 0.614 & 0.619 & 0.663 & 0.660 & 0. & 0.584 & 0.576 & & & 0. & & & .587 \\
\hline$S D D B$ & 595 & 0.594 & 0.637 & 0.736 & 0.795 & 0.836 & 0.830 & 0.559 & 0.580 & 0.688 & 0.837 & 0.883 & & 0.798 \\
\hline$S D D B_{A R}$ & 594 & 0.588 & 0.636 & 0.739 & 0.800 & 0.810 & 0.681 & 0.579 & 0.577 & 0.702 & 0.822 & 36 & & .495 \\
\hline Siev & 592 & 0.611 & 0.624 & 0.662 & 0.669 & 0.6 & 0.564 & 0.635 & 0.7 & 0.722 & 0.764 & 52 & & .678 \\
\hline Sieve $_{H}$ & 0.649 & 0.642 & 0.666 & 0.672 & 0.707 & 0.691 & 0.604 & 0.793 & 0.853 & 0.868 & 0.878 & 0.904 & 0.896 & 0.663 \\
\hline$M B B_{b_{1}}^{F L}$ & 0.862 & 0.868 & 0.879 & 0.867 & 0.894 & 0.883 & 0.802 & 0.864 & 0.889 & 0.884 & 0.891 & 0.895 & 0.904 & 0.850 \\
\hline$M B B_{b_{2}}^{F D}$ & 0.861 & 0.872 & 0.886 & 0.878 & 0.899 & 0.872 & 0.801 & 0.856 & 0.887 & 0.882 & 0.880 & 0.878 & 0.896 & 0.847 \\
\hline$M B B_{b_{3}}^{\sigma_{2} D B}$ & 0.836 & 0.837 & 0.858 & 0.835 & 0.872 & 0.854 & 0.779 & 0.845 & 0.863 & 0.869 & 0.861 & 0.855 & 0.879 & 0.824 \\
\hline$M B B_{b^{*}}^{F} D B$ & 0.838 & 0.839 & 0.848 & 0.841 & 0.859 & 0.848 & 0.770 & 0.830 & 0.853 & 0.853 & 0.855 & 0.864 & 0.869 & 0.823 \\
\hline$S B B_{b_{1}}^{F D B}$ & 0.843 & 0.858 & 0.872 & 0.850 & 0.885 & 0.863 & 0.788 & 0.859 & 0.876 & 0.881 & 0.873 & 0.885 & 0.890 & 0.830 \\
\hline$S B B_{b_{2}}^{F} D B$ & 0.849 & 0.855 & 0.865 & 0.855 & 0.880 & 0.860 & 0.776 & 0.845 & 0.876 & 0.870 & 0.862 & 0.868 & 0.881 & 0.829 \\
\hline$S E$ & 0.828 & 0.836 & 0.852 & 0.838 & 0.864 & 0.850 & 0.785 & 0.828 & 0.863 & 0.845 & 0.845 & 0.864 & 0.862 & 0.822 \\
\hline$S B B_{b^{*}}^{F D B}$ & 0.839 & 0.846 & 0.853 & 0.842 & 0.851 & 0.861 & 0.771 & 0.842 & 0.860 & 0.864 & 0.852 & 0.857 & 0.875 & 0.818 \\
\hline$S D D B_{M A}^{F D B}$ & 0.836 & 0.836 & 0.843 & 0.836 & 0.861 & 0.856 & 0.775 & 0.845 & 0.864 & 0.864 & 0.861 & 0.864 & 0.877 & 0.831 \\
\hline$S D D B_{A R}^{F D B}$ & 0.832 & 0.841 & 0.845 & 0.834 & 0.867 & 0.863 & 0.772 & 0.843 & 0.865 & 0.860 & 0.862 & 0.867 & 0.876 & 0.830 \\
\hline Sieve $_{A I C}^{F D B}$ & 0.710 & 0.711 & 0.737 & 0.749 & 0.756 & 0.737 & 0.673 & 0.786 & 0.812 & 0.799 & 0.783 & 0.809 & 0.812 & 0.779 \\
\hline Sieve $_{H}^{F D B}$ & 0.659 & 0.658 & 0.681 & 0.690 & 0.711 & 0.688 & 0.609 & 0.818 & 0.851 & 0.841 & 0.825 & 0.832 & 0.850 & 0.792 \\
\hline
\end{tabular}

Table 9: Empirical Coverage Rates for $S_{T}^{d}$ using $A R F I M A(0, d, 1), \theta=0.8$ DGPs.

Notes: The top panel of the table shows the coverage rates for the bootstrap methods outlined in Section 2 directly applied to the original levels series. The bottom panel shows

the coverage rates for the bootstrap methods using the suggested FDB methodology. 


\begin{tabular}{|c|c|c|c|c|c|c|c|c|c|c|c|c|c|c|}
\hline \multicolumn{15}{|c|}{$\operatorname{ARFIMA}(0, d, 1), \theta=-0.8, S_{T}^{d}$} \\
\hline \multirow[b]{3}{*}{ Bootstrap \dd } & \multicolumn{14}{|c|}{$E L W$} \\
\hline & \multicolumn{7}{|c|}{$T=120$} & \multicolumn{7}{|c|}{$T=500$} \\
\hline & 0 & 0.15 & 0.25 & 0.35 & 0.45 & 0.49 & 0.75 & 0 & 0.15 & 0.25 & 0.35 & 0.45 & 0.49 & 0.75 \\
\hline$M B B_{b_{1}}$ & 0.203 & 0.315 & 0.383 & 0.461 & 0.518 & 549 & 0.608 & 0.344 & 0.531 & 0.612 & 0.596 & 0.528 & 0.499 & 189 \\
\hline$M B$ & 0.203 & 0.315 & 0.375 & 0.460 & 0.524 & 0.565 & 0.614 & 0.347 & 0.531 & 0.601 & 0.594 & 0.531 & 0.501 & 0.195 \\
\hline$M B B_{b_{3}}$ & 0.235 & 0.342 & 0.398 & 0.479 & 0.533 & 0.576 & 0.643 & 0.372 & 0.549 & 0.613 & 0.606 & 0.536 & 0.526 & 0.224 \\
\hline$M B B_{b^{*}}$ & 0.369 & 0.466 & 0.472 & 0.524 & 0.552 & 0.588 & 0.687 & 0.503 & 0.619 & 0.672 & 0.669 & 0.573 & 0.525 & 0.789 \\
\hline$S B B_{b_{1}}$ & 0.203 & 0.311 & 0.370 & 0.466 & 0.524 & 0.575 & 0.643 & 0.342 & 0.523 & 0.596 & 0.598 & 0.540 & 0.511 & 0.209 \\
\hline$S B B_{b_{2}}$ & 0.204 & 0.315 & 0.373 & 0.456 & 0.527 & 0.568 & 0.647 & 0.351 & 0.532 & 0.611 & 0.613 & 0.571 & 0.547 & 0.288 \\
\hline$S B B_{b_{3}}$ & 0.243 & 0.337 & 0.403 & 0.483 & 0.541 & 0.596 & 0.686 & 0.370 & 0.553 & 0.632 & 0.648 & 0.623 & 0.604 & 0.441 \\
\hline$S B B_{b^{*}}$ & 0.338 & 0.418 & 0.469 & 0.515 & 0.554 & 0.579 & 0.695 & 0.518 & 0.603 & 0.689 & 0.696 & 0.668 & 0.604 & 0.810 \\
\hline$S D I$ & 0.454 & 0.477 & 0.446 & 0.490 & 0.543 & 0.555 & 0.672 & 0.524 & 0.574 & 0.604 & 0.590 & 0.525 & 0.494 & 0.739 \\
\hline$S D D B_{A F}$ & 0.462 & 0.470 & 0.448 & 0.492 & 0.531 & 0.562 & 0.648 & 0.519 & 0.566 & 0.599 & 0.594 & 0.525 & 0.507 & 0.387 \\
\hline Siev & 0.205 & 0.279 & 0.310 & 0.387 & 0.410 & 0.435 & 0.594 & 0.410 & 0.542 & 0.612 & 0.636 & 0.709 & 0.730 & 687 \\
\hline & 0.338 & 0.378 & 0.361 & 0.408 & 0.423 & 0.412 & 0.468 & 0.586 & 0.659 & 0.654 & 0.676 & 0.666 & 0.657 & 0.661 \\
\hline$M B$ & 0.548 & 0.592 & 0.592 & 0.593 & 0.573 & 0.576 & 0.542 & 0.744 & 0.736 & 0.762 & 0.736 & 0.718 & 0.714 & 0.722 \\
\hline$M B B_{b_{D}}^{D_{1} D B}$ & 0.542 & 0.578 & 0.589 & 0.582 & 0.573 & 0.586 & 0.549 & 0.761 & 0.730 & 0.756 & 0.749 & 0.746 & 0.711 & 0.733 \\
\hline$M B B_{b_{3}}^{F} D B$ & 0.543 & 0.604 & 0.596 & 0.618 & 0.591 & 0.618 & 0.576 & 0.751 & 0.760 & 0.769 & 0.768 & 0.756 & 0.756 & 0.732 \\
\hline$M B B_{b^{*}}^{F^{3}} D B$ & 0.525 & 0.583 & 0.574 & 0.581 & 0.582 & 0.603 & 0.569 & 0.724 & 0.723 & 0.735 & 0.743 & 0.721 & 0.701 & 0.707 \\
\hline$S B B_{b_{1}}^{F}$ & 0.530 & 0.576 & 0.583 & 0.600 & 0.570 & 0.596 & 0.560 & 0.741 & 0.735 & 0.744 & 0.733 & 0.719 & 0.716 & 0.723 \\
\hline$S B B$ & 0.536 & 0.581 & 0.574 & 0.584 & 0.580 & 0.582 & 0.560 & 0.761 & 0.740 & 0.768 & 0.743 & 0.738 & 0.723 & 0.714 \\
\hline$S B B_{b_{2}}^{F_{2} D B}$ & 0.535 & 0.587 & 0.597 & 0.601 & 0.602 & 0.604 & 0.569 & 0.752 & 0.737 & 0.762 & 0.740 & 0.739 & 0.736 & 0.730 \\
\hline$S B B_{b^{*}}^{F^{*} D B}$ & 0.528 & 0.589 & 0.567 & 0.592 & 0.589 & 0.603 & 0.561 & 0.722 & 0.718 & 0.735 & 0.733 & 0.722 & 0.701 & 0.685 \\
\hline$S D D B_{M A}^{F D B}$ & 0.504 & 0.562 & 0.536 & 0.560 & 0.557 & 0.549 & 0.524 & 0.688 & 0.704 & 0.714 & 0.713 & 0.713 & 0.692 & 0.688 \\
\hline$S D D B_{A R}^{F D B}$ & 0.514 & 0.553 & 0.538 & 0.565 & 0.554 & 0.556 & 0.516 & 0.702 & 0.701 & 0.721 & 0.715 & 0.717 & 0.699 & 0.686 \\
\hline & 82 & 0.409 & 0.405 & 0.409 & 0.401 & 0.398 & 0.347 & 0.715 & 0.711 & 0.729 & 0.704 & 0.691 & 0.684 & 0.659 \\
\hline Sie & 0.370 & 0.424 & 0.393 & 0.426 & 0.431 & 0.409 & 0.344 & 0.669 & 0.676 & 0.665 & 0.667 & 0.643 & 0.640 & 0.617 \\
\hline
\end{tabular}

Table 10: Empirical Coverage Rates for $S_{T}^{d}$ using $A R F I M A(0, d, 1), \theta=-0.8$ DGPs.

Notes: The top panel of the table shows the coverage rates for the bootstrap methods outlined in Section 2 directly applied to the original levels series. The bottom panel shows

the coverage rates for the bootstrap methods using the suggested FDB methodology. 


\title{
A Generalised Fractional Differencing Bootstrap for Long Memory Processes
}

\author{
Supplementary Appendix
}

by

G. Kapetanios, F. Papailias and A.M.R. Taylor 


\section{A.1 Introduction}

This supplement to our paper "A Generalised Fractional Differencing Bootstrap for Long Memory Processes" has four sections. The first section contains a proof of Theorem 1. The second section contains the modification of Algorithm 3 needed to allow for an unknown mean in the data. The third section provides additional computational details showing how we conducted the simulation experiments reported in the paper and how we implemented the bootstrap procedures. The final section contains additional simulation results. Equation references (A. $n$ ) for $n \geq 1$ refer to equations in this supplementary appendix and other equation references are to the main paper. An additional reference is included at the end of the supplement.

\section{A.2 Proof of Theorem 1}

Our proof shares some similarities with the treatment of Wright (1995) and is presented below. In particular, as in Wright (1995), we want to show that operations in the underlying $I(0)$ series can be translated to $I(d)$ space provided that a consistent estimator of $d$ is used. Below, we sketch our proof before providing formal details.

1. Let $Y_{t}$ be as in (1) and $X_{t}^{*}$ be obtained by the FDB using the true value of $d$ in the differencing and cumulation steps of Algorithm 3. We first must show that the distribution of a statistic based on $Y_{t}$ and the distribution of the same statistic based on $X_{t}^{*}$, are asymptotically equal.

2. Next, let $Y_{t}^{*}$ be constructed similarly to $X_{t}^{*}$ using Algorithm 3, but $\widehat{d}$ is used for the differencing and cumulation in Steps 3 and 5 of the algorithm. $\widehat{d}$ is obtained using an estimator which satisfies Assumption 2. We must show that the distribution of a statistic based on $X_{t}^{*}$ and the same statistic based on $Y_{t}^{*}$, are asymptotically equal. The mapping from the original data to the bootstrap should be a function of $d$. In other words, bootstrapping in the short memory space should not be stochastic; i.e. if we were to resample with replacement the short memory series, we should do it in the same way for $X_{t}^{*}$ and $Y_{t}^{*}$ - the only difference should be the use of $d$ and $\widehat{d}$. Therefore, the two statistics differ only because $\widehat{d}$ and $d$ differ, but as $\widehat{d}$ converges to $d$ so should the distribution of the statistics. We further subdivide this step into two by specifying that in one step, $\widehat{d}$ is used for differencing and $d$ for cumulation and the reverse for the other step.

In summary, to proceed with the proof of Theorem 1 we consider the following series.

- $Y_{t}$ is the original fractionally integrated series, described in (1). $Y_{t}$ is $I(d)$ and we calculate the statistic $S_{T}=S_{T}\left(Y_{1}, . ., Y_{T}\right)$ which satisfies Assumption 3. 
- $X_{t}^{*}$ is the FDB resample in the theoretical case where $d$ is known and used in the differencing and cumulation steps of Algorithm 3. $X_{t}^{*}$ is $I(d)$ and we calculate the statistic $S_{T}^{*(d, d)}=S_{T}\left(X_{1}^{*}, \ldots, X_{T}^{*}\right)$.

- $Y_{t}^{*}$ is the FDB resample exactly as described in Algorithm 3, using $\widehat{d}$ for differencing and cumulation. $\widehat{d}$ is obtained using an estimator which satisfies Assumption 2 and $Y_{t}^{*}$ is $I(d)$. Using $Y_{t}^{*}$ we calculate the statistic $S_{T}^{*(\widehat{d}, \widehat{d})}=S_{T}\left(Y_{1}^{*}, . ., Y_{T}^{*}\right)$.

- Finally, $H_{t}^{*}$ is the FDB resample using $\widehat{d}$ for differencing and $d$ for cumulation. $H_{t}^{*}$ is $I(2 d-\widehat{d})$ and we calculate the statistic $S_{T}^{*(\widehat{d}, d)}=S_{T}\left(H_{1}^{*}, . ., H_{T}^{*}\right)$.

Denote the distribution of the statistic under $\mathcal{P}_{\mathbf{Y}_{T}}$ by $F_{S_{T}}$ and $F_{S_{T}^{*(d, d)}}, F_{S_{T}^{*(\widehat{d}, d)}}$ and $F_{S_{T}^{*(\widehat{d}, d)}}$ are corresponding approximations to the true distribution of the statistic under $\mathcal{P}_{\mathbf{X}_{t}^{*}}, \mathcal{P}_{\mathbf{Y}_{t}^{*}}$ and $\mathcal{P}_{\mathbf{H}_{t}^{*}}$ respectively.

In what follows we use the operator $\Delta_{+}^{d}$, which is the inverse of $\Delta_{+}^{-d}$ in (1), defined for a generic variable $y_{t}$ as $\Delta_{+}^{d}:=\Delta^{d} y_{t} \mathbb{I}(t \geq 1)=\sum_{n=0}^{t-1} \alpha_{n}(d) y_{t-n}$ where $\mathbb{I}(\cdot)$ denotes the indicator function and $\alpha_{n}(d):=\frac{\Gamma(n-d)}{\Gamma(-d) \Gamma(n+1)}$ denotes the coefficients in the binomial expansion of $(1-z)^{d}$.

Proof. Using Assumption 3 we have that,

$$
\begin{aligned}
& \left\{\eta\left(F_{S_{T}^{*(\widehat{d}, \widehat{d})}}, F_{S_{T}}\right)\right\}^{2} \leq\left\{\eta\left(F_{S_{T}^{*(\widehat{d}, \widehat{d})}}, F_{S_{T}^{*(d, d)}}\right)\right\}^{2}+\left\{\eta\left(F_{S_{T}^{*(d, d)}}, F_{S_{T}}\right)\right\}^{2} \\
& \leq\left\{\eta\left(F_{S_{T}^{*(\widehat{d}, \widehat{d})}}, F_{S_{T}^{*(\widehat{d}, d)}}\right)\right\}^{2}+\left\{\eta\left(F_{S_{T}^{*(\widehat{d}, d)}}, F_{S_{T}^{*(d, d)}}\right)\right\}^{2}+\left\{\eta\left(F_{S_{T}^{*(d, d)}}, F_{S_{T}}\right)\right\}^{2} .
\end{aligned}
$$

The last term of the right hand side of the above is straightforward, since the true $d$ is used. Therefore, for valid short memory bootstrap methods, $\eta\left(F_{S_{T}^{*(d, d)}}, F_{S_{T}}\right)=o_{p}(1)$. We focus on the first and second terms. From the definition of Mallows metric, using Assumption 3 and applying the Cauchy-Schwartz inequality twice we have,

$$
\begin{gathered}
\left\{\eta\left(F_{S_{T}^{*(\widehat{d}, \widehat{d})}}, F_{S_{T}^{*}(\widehat{d}, d)}\right)\right\}^{2} \leq E\left[E^{*}\left[\left\|S_{T}^{*(\widehat{d}, \widehat{d})}-S_{T}^{*(\widehat{d}, d)}\right\|^{2}\right]\right] \\
\leq\left\{T^{-1} \sum_{t=1}^{T} E\left[E^{*}\left[\left(B_{t}\left(\boldsymbol{H}_{T}^{*}, \boldsymbol{Y}_{T}^{*}\right)\right)^{2}\right]\right]\right\}\left\{T^{-\left(1+\max \left\{0, d-\frac{1}{2}\right\}\right)} \sum_{t=1}^{T} E\left[E^{*}\left[\left(H_{t}^{*}-Y_{t}^{*}\right)^{2}\right]\right]\right\} \\
\leq\left\{\lim _{T \rightarrow \infty} \sup T^{-1} \sum_{t=1}^{T} E\left[E^{*}\left[\left(B_{t}\left(\boldsymbol{H}_{T}^{*}, \boldsymbol{Y}_{T}^{*}\right)\right)^{2}\right]\right]\right\}\left\{T^{-\left(1+\max \left\{0, d-\frac{1}{2}\right\}\right)} \sum_{t=1}^{T} E\left[E^{*}\left[\left(H_{t}^{*}-Y_{t}^{*}\right)^{2}\right]\right]\right\} \\
=\left\{\lim _{T \rightarrow \infty} \sup T^{-1} \sum_{t=1}^{T} E\left[E^{*}\left[\left(B_{t}\left(\boldsymbol{H}_{T}^{*}, \boldsymbol{Y}_{T}^{*}\right)\right)^{2}\right]\right]\right\}\left\{\lim _{T \rightarrow \infty} \sup T^{-\left(1+\max \left\{0, d-\frac{1}{2}\right\}\right)} \sum_{t=1}^{T} E\left[E^{*}\left[\left(H_{t}^{*}-Y_{t}^{*}\right)^{2}\right]\right]\right\} .
\end{gathered}
$$


Given that there exists a function $B_{t}\left(\boldsymbol{H}_{T}^{*}, \boldsymbol{Y}_{T}^{*}\right)$ such that the first limit is finite, we are interested in the last term of the right hand side of the above. Hence, we need to prove that,

$$
\lim _{T \rightarrow \infty} \sup T^{-\left(1+\max \left\{0, d-\frac{1}{2}\right\}\right)} \sum_{t=1}^{T} E\left[E^{*}\left[\left(H_{t}^{*}-Y_{t}^{*}\right)^{2}\right]\right]=0 .
$$

Using (1) we have that,

$$
H_{t}^{*}=\Delta_{+}^{-d} u_{t}^{*} \text { and } Y_{t}^{*}=\Delta_{+}^{-\hat{d}} u_{t}^{*},
$$

where $u_{t}^{*}$ is the resampled $I(0)$ process. Following similar steps to those in Wright (1995),

$$
\begin{gathered}
T^{-\max \left\{0, d-\frac{1}{2}\right\}}\left(H_{t}^{*}-Y_{t}^{*}\right)=T^{-\max \left\{0, d-\frac{1}{2}\right\}}\left(\Delta_{+}^{-d} u_{t}^{*}-\Delta_{+}^{-\hat{d}} u_{t}^{*}\right)= \\
T^{-\max \left\{0, d-\frac{1}{2}\right\}}\left((1-L)^{-d} \sum_{j=1}^{t-1} \alpha_{j}(\widehat{d}-d) u_{t-j}^{*}\right)= \\
T^{-\max \left\{0, d-\frac{1}{2}\right\}}\left((1-L)^{-d}\left((\widehat{d}-d) \sum_{j=1}^{t-1} \alpha_{j}^{\prime}(\widehat{d}-d) u_{t-j}^{*}+(\widehat{d}-d)^{2} \sum_{j=1}^{t-1} \alpha_{j}^{\prime \prime}(\widehat{d}-d) u_{t-j}^{*}\right)\right),
\end{gathered}
$$

since,

$$
\alpha_{j}(\widehat{d}-d)=(\widehat{d}-d) \alpha_{j}^{\prime}(\widehat{d}-d)+(\widehat{d}-d)^{2} \alpha_{j}^{\prime \prime}(\widehat{d}-d),
$$

where $\alpha_{j}^{\prime}$ and $\alpha_{j}^{\prime \prime}$ denote the first and second derivative of $\alpha_{j}(\cdot)$ respectively. Using (A.3) and (A.4), it suffices to prove that

$$
\begin{aligned}
\lim _{T \rightarrow \infty} \sup & \sum_{t=1}^{T} E\left[E ^ { * } \left[\left((\widehat{d}-d) \sum_{j=1}^{t-1} \alpha_{j}^{\prime}(\widehat{d}-d) u_{t-j}^{*}\right.\right.\right. \\
& \left.\left.\left.+(\widehat{d}-d)^{2} \sum_{j=1}^{t-1} \alpha_{j}^{\prime \prime}(\widehat{d}-d) u_{t-j}^{*}\right)^{2}\right]\right]=0 .
\end{aligned}
$$

Under Assumption 2, the variance of $(\widehat{d}-d)$ goes to zero as $T \rightarrow \infty$,

$$
\lim _{T \rightarrow \infty} \sup E\left[E^{*}\left[(\widehat{d}-d)^{2}\right]\right]=0,
$$

see for example Robinson (1995) and Shimotsu and Phillips (2006). The first limit of (A.6) 
can be written as

$$
\begin{gathered}
\lim _{T \rightarrow \infty} \sup \sum_{t=1}^{T} E\left[E^{*}\left[\left((\widehat{d}-d) \sum_{j=1}^{t-1} \alpha_{j}^{\prime}(\widehat{d}-d) u_{t-j}^{*}\right)^{2}\right]\right] \\
\leq\left\{\lim _{T \rightarrow \infty} \sup E\left[E^{*}\left[(\widehat{d}-d)^{2}\right]\right]\right\}\left\{\lim _{T \rightarrow \infty} \sup \sum_{t=1}^{T} E\left[E^{*}\left[\left(\sum_{j=1}^{t-1} \alpha_{j}^{\prime}(\widehat{d}-d) u_{t-j}^{*}\right)^{2}\right]\right]\right\}
\end{gathered}
$$

where the second limit is finite. Using (A.7) and (A.8), (A.6) is proved.

Similarly,

$$
\begin{gathered}
\left\{\eta\left(F_{S_{T}^{*(\widehat{d}, d)}}, F_{S_{T}^{*(d, d)}}\right)\right\}^{2} \leq E\left[E^{*}\left[\left\|S_{T}^{*(\widehat{d}, d)}-S_{T}^{*(d, d)}\right\|^{2}\right] \leq\right. \\
\left.\left\{T^{-1} \sum_{t=1}^{T} E\left[E^{*}\left[\left(B_{t}\left(\boldsymbol{X}_{T}^{*}, \boldsymbol{H}_{T}^{*}\right)\right)^{2}\right]\right]\right\} T^{-\left(1+\max \left\{0, d-\frac{1}{2}\right\}\right)} \sum_{t=1}^{T} E\left[E^{*}\left[\left(X_{t}^{*}-H_{t}^{*}\right)^{2}\right]\right]\right\} \\
\leq\left\{\lim _{T \rightarrow \infty} \sup T^{-1} \sum_{t=1}^{T} E\left[E^{*}\left[\left(B_{t}\left(\boldsymbol{X}_{T}^{*}, \boldsymbol{H}_{T}^{*}\right)\right)^{2}\right]\right]\right\}\left\{T^{-\left(1+\max \left\{0, d-\frac{1}{2}\right\}\right)} \sum_{t=1}^{T} E\left[E^{*}\left[\left(X_{t}^{*}-H_{t}^{*}\right)^{2}\right]\right]\right\} \\
\leq\left\{\lim _{T \rightarrow \infty} \sup T^{-1} \sum_{t=1}^{T} E\left[E^{*}\left[\left(B_{t}\left(\boldsymbol{X}_{T}^{*}, \boldsymbol{H}_{T}^{*}\right)\right)^{2}\right]\right]\right\}\left\{\lim _{T \rightarrow \infty} \sup T^{-\left(1+\max \left\{0, d-\frac{1}{2}\right\}\right)} \sum_{t=1}^{T} E\left[E^{*}\left[\left(X_{t}^{*}-H_{t}^{*}\right)^{2}\right]\right]\right\}
\end{gathered}
$$

and hence we need to prove that,

$$
\lim _{T \rightarrow \infty} \sup T^{-\left(1+\max \left\{0, d-\frac{1}{2}\right\}\right)} \sum_{t=1}^{T} E\left[E^{*}\left[\left(X_{t}^{*}-H_{t}^{*}\right)^{2}\right]\right]=0 .
$$

which can be proven similarly to the result in (A.3), completing the proof. 


\section{A.3 Modification of Algorithm 3 to Allow for an Un- known Mean}

\section{Algorithm 4.}

Step 1. Given the data $Y_{t}, t=1, \ldots, T$, calculate the statistic of interest $S_{T}=S_{T}\left(Y_{1}, . ., Y_{T}\right)$.

Step 2. Estimate $\mu$ using (5) and construct the demeaned series, $\mathcal{Y}_{t}:=Y_{t}-\widehat{\mu}(d)$.

Step 3. Based on $\mathcal{Y}_{t}$, obtain an estimate, $\widehat{d}$, of the long memory parameter, $d$, which satisfies Assumption 2.

Step 4. [Differencing] Filter $\mathcal{Y}_{t}$ using the fractional differencing operator, $\Delta_{+}^{\widehat{d}}$, and obtain,

$$
\widehat{u}_{t}:=\Delta_{+}^{\widehat{d}} \mathcal{Y}_{t}
$$

Step 5. [Resampling] Apply any asymptotically valid bootstrap for weakly autocorrelated series, as described separately below, and obtain a resample for $\widehat{u}_{t}$, denoted by $\widehat{u}_{t}^{*}$.

(a) Sieve AR Bootstrap

i. Fit an $A R(h)$ to $\widehat{u}_{t}$, let those estimates be denoted by $\widehat{\varphi}:=\left(\widehat{\varphi}_{1}, \ldots, \widehat{\varphi}_{h}\right)^{\prime}$, and evaluate the corresponding residuals $\widehat{\varepsilon}_{t}:=\left(\widehat{u}_{t}-\bar{u}\right)-\sum_{j=1}^{h} \widehat{\varphi}_{j}\left(\widehat{u}_{t-j}-\bar{u}\right)$ and the associated residual variance estimate, $\widehat{\sigma}_{h_{\varepsilon}}^{2} \cdot \bar{u}$ denotes the sample mean of $\widehat{u}_{t}$.

ii. Using $\widehat{\varepsilon}_{t}$, calculate the standardised residuals, $\widetilde{\varepsilon}_{t}:=\frac{\widehat{\varepsilon}_{t}-\bar{\varepsilon}}{s_{\bar{\varepsilon}}}$, where $\bar{\varepsilon}$ and $s_{\bar{\varepsilon}}$ denote the sample mean and standard deviation of $\widehat{\varepsilon}_{t}$, respectively.

iii. Construct a random sample with replacement for $\widetilde{\varepsilon}_{t}$ denoted by $\widetilde{\varepsilon}^{*}:=\left(\widetilde{\varepsilon}_{1}^{*}, \ldots, \widetilde{\varepsilon}_{T}^{*}\right)^{\prime}=$ $\left(\widetilde{\varepsilon}_{I_{1}}, \ldots, \widetilde{\varepsilon}_{I_{T}}\right)^{\prime}$ with $I_{1}, \ldots, I_{T}$ being i.i.d. random variables with discrete uniform distribution on $\{1, \ldots, T\}$. Alternatively one could use the wild bootstrap in place of the i.i.d. resampling scheme.

$i v$. The sieve AR bootstrap data observations are generated via the recursion,

$$
\widehat{u}_{t}^{*}=\bar{u}+\sum_{j=1}^{h} \widehat{\varphi}_{j}\left(\widehat{u}_{t-j}^{*}-\bar{u}\right)+\widehat{\sigma}_{h_{\varepsilon}} \widetilde{\varepsilon}_{t}^{*},
$$

initialised at $\widehat{u}_{1-j}^{*}=\widehat{u}_{\tau-j+1}, j=1, \ldots, h$ where $\tau$ has the discrete uniform distribution on $\{h, \ldots, T\}$.

(b) $M B B$

i. Construct the de-meaned series of $\widehat{u}_{t}$, defined as $v_{t}:=\left(\widehat{u}_{1}-\bar{u}, \ldots ., \widehat{u}_{T}-\bar{u}\right)^{\prime}$, and divide it into $M$ overlapping blocks of $b$ observations, with $M:=T-b+1$. 
Denote the collection of blocks by $\left\{B_{1}, \ldots, B_{M}\right\}$ and let $I_{1}, \ldots, I_{T / b}$ be i.i.d. random variables with discrete uniform distribution on $\{1, \ldots, M\}$.

ii. A MBB resample for $v_{t}$ is then obtained with replacement from the collection of blocks, $v_{t}^{*}:=\left(B_{I_{1}}, \ldots, B_{I_{T / b}}\right)^{\prime}$.

iii. Obtain the corresponding resample for $\widehat{u}_{t}$ as,

$$
\widehat{u}_{t}^{*}=v_{t}^{*}+\bar{u} .
$$

(c) $S B B$

i. Construct the de-meaned series of $\widehat{u}_{t}$, defined as $v_{t}:=\left(\widehat{u}_{1}-\bar{u}, \ldots, \widehat{u}_{T}-\bar{u}\right)^{\prime}$. $S B B$ uses a random block length for $b$. Let $L_{1}, L_{2}, \ldots$ be a sequence of i.i.d. random variables having geometric distribution, so that the probability of the event $\left\{L_{i}=\eta\right\} \quad$ is $(1-p)^{\eta-1}$ for $\eta=1,2, \ldots$ and $p$ is a fixed number in $[0,1]$. Denote the collection of blocks by $\left\{B_{L_{1}}, B_{L_{2}}, \ldots\right\}$. The indicator $L_{\eta}$ denotes the length of the relevant block. Let $I_{1}, I_{2}, \ldots$ be a sequence of i.i.d. variables which have discrete uniform distribution on $\{1, \ldots, T\}$.

ii. A SBB resample for $v_{t}$ is obtained by random draws with replacement from the collection of blocks, $v_{t}^{*}:=\left(B_{L_{1}} B_{L_{2}}, \ldots,\right)^{\prime}$ until $t=T$.

iii. Obtain the corresponding resample for $\widehat{u}_{t}$ as,

$$
\widehat{u}_{t}^{*}=v_{t}^{*}+\bar{u} .
$$

(d) $S D D B-M A$

i. Consider the MA representation of $u_{t}, u_{t}=\varepsilon_{t}+\sum_{j=1}^{\infty} c_{j} \varepsilon_{t-j}$, and the spectral density of $u_{t}, f_{u}$. Obtain an estimate of $f_{u}$ using $\widehat{u}_{t}$ denoted by $\widehat{f}_{\widehat{u}}$.

ii. Compute the Fourier coefficients of $\log \left(\widehat{f}_{\widehat{u}}\right)$ given by $\widehat{a}_{k}:=\frac{1}{2 \pi} \int_{0}^{2 \pi} \log \left\{\widehat{f}_{\widehat{u}}(\omega)\right\} e^{-i k \omega} d \omega$ for $k=0,1,2, \ldots$

iii. Let $\widehat{\sigma}_{a}^{2}:=2 \pi e^{\widehat{a}_{0}}$ and compute the coefficients $\widehat{c}_{k}$, using $\widehat{c}_{k+1}=\sum_{j=0}^{k}\left(\frac{1-j}{k+1}\right) \widehat{a}_{k+1-j} \widehat{c}_{j}$ for $k=0,1,2, \ldots$ and starting value $\widehat{c}_{0}=1$.

iv. Generate i.i.d. pseudo-innovations $\left(\varepsilon_{1}^{*}, \ldots, \varepsilon_{T}^{*}\right)^{\prime}$ with mean 0 and variance $\widehat{\sigma}_{a}^{2}$.

$v$. Generate the pseudo-time series $\left(\widehat{u}_{1}^{*}, \ldots ., \widehat{u}_{T}^{*}\right)^{\prime}$ obtained by,

$$
\widehat{u}_{t}^{*}=\bar{u}+\sum_{j=0}^{\infty} \widehat{c}_{j} \varepsilon_{t-j}^{*}
$$

(e) $S D D B-A R$

i. Consider the $A R$ representation of $u_{t}, u_{t}=\sum_{j=1}^{\infty} b_{j} u_{t-j}+\varepsilon_{t}, \sum_{j=1}^{\infty}\left|b_{j}\right|<\infty$, 
and the spectral density of $u_{t}, f_{u}$. Obtain an estimate of $f_{u}$ using $\widehat{u}_{t}$ denoted by $\widehat{f}_{\widehat{u}}$.

ii. Compute the Fourier coefficients of $\log \left(\widehat{f}_{\widehat{u}}\right)$ given by $\widehat{a}_{k}:=\frac{1}{2 \pi} \int_{0}^{2 \pi} \log \left\{\widehat{f}_{\widehat{u}}(\omega)\right\} e^{-i k \omega} d \omega$ for $k=0,1,2, \ldots$

iii. Let $\widehat{\sigma}_{a}^{2}:=2 \pi e^{\widehat{a}_{0}}$ and compute the coefficients $\widehat{b}_{k}$, using the recursive formula starting with $\widehat{b}_{0}=-1$ and $\widehat{b}_{k+1}=-\sum_{j=0}^{k}\left(\frac{1-j}{k+1}\right) \widehat{a}_{k+1-j} \widehat{b}_{j}$ for $k=0,1,2, \ldots$.

iv. Generate i.i.d. pseudo-innovations $\left(\varepsilon_{1}^{*}, \ldots, \varepsilon_{T}^{*}\right)^{\prime}$ with mean 0 and variance $\widehat{\sigma}_{a}^{2}$.

v. Generate the pseudo-time series $\left(\widehat{u}_{1}^{*}, \ldots ., \widehat{u}_{T}^{*}\right)^{\prime}$ obtained by,

$$
\widehat{u}_{t}^{*}=\bar{u}+\sum_{j=1}^{t-1} \widehat{b}_{j}\left(\widehat{u}_{t-j}^{*}-\bar{u}\right)+\varepsilon_{t}^{*}
$$

Step 6. [Cumulation]Apply the fractional cumulating operator $\Delta_{+}^{-\widehat{d}}$ to $\widehat{u}_{t}^{*}$ and obtain the resampled data $\mathcal{Y}_{t}^{*}=\Delta_{+}^{-} \widehat{d}_{t}^{*}$. Finally, the corresponding resample to the original data is obtained by,

$$
Y_{t}^{*}:=\widehat{\mu}(d)+\mathcal{Y}_{t}^{*}
$$

Step 7. Calculate $S_{T}^{*}=S_{T}\left(Y_{1}^{*}, . ., Y_{T}^{*}\right)$ which is the bootstrap analogue to $S_{T}$.

Step 8. Repeat Steps 5 to 7, to construct B independent bootstrap samples and bootstrap statistics, denoted $Y_{l, T}^{*}$ and $S_{l, T}^{*}, l=1, \ldots, B$, respectively, and collect $S_{B, T}^{*}:=\left(S_{1, T}^{*}, \ldots, S_{B, T}^{*}\right)^{\prime}$. Approximate $F_{S_{T}}(\mathbf{s})$ by the bootstrap distribution function $\bar{F}_{S_{B, T}^{*}}(\mathbf{s})=\frac{1}{B} \sum_{l=1}^{B} I\left(S_{B, T}^{*} \leq\right.$ s).

\section{A.4 Simulation Algorithm and Coverage Rates}

Below we outline how we conduct the simulation experiments and implement the bootstrap procedures.

\section{Algorithm 5.}

Step 1. For the ith simulation, with $i=1,2, \ldots, R$ and $R=1000$, generate sample data for $u_{t}$, $t=1,2, \ldots T$, which follows an $\operatorname{ARMA}(p, q)$ model with standard normal innovations; the starting value is always set to be $u_{1}=0$. The settings for $T, p$ and $q$ are as described in the main text.

Step 2. Then, apply the inverse of the Fast Fractional Difference (FFD), introduced by Jensen and Nielsen (2014), and generate $Y_{t}=\mu+\Delta_{+}^{-d} u_{t}$ setting $\mu=0$. Therefore,

$$
Y_{t}:=\Delta_{+}^{-d} u_{t}
$$


The values of $d$ considered are as given in the main text. Calculate the statistic of interest $S_{T}=S_{T}\left(Y_{1}, . ., Y_{T}\right)$.

Step 3. Construct $B=199$ bootstrap resamples for $Y_{t}$, denoted by $Y_{l, T}^{*}, l=1, \ldots, B$, using two approaches:

(a) Ignoring the presence of long memory and applying the sieve $A R$ bootstrap, $M B B$, $S B B, S D D B$ using the $M A$ or $A R$ representation directly to $Y_{t}$; exactly as discussed in Algorithm 4. The AR order, $h$, for the sieve is obtained: (i) using AIC with maximum order $H:=\left\lfloor(\log T)^{2}\right\rfloor$, and (ii) setting $h=\left\lfloor(\log T)^{2}\right\rfloor$. The latter is $h=23$ for $T=120$ and $h=39$ for $T=500$. We use OLS to estimate the $A R$ parameters in the sieve bootstrap.

(b) Employing FDB and applying Algorithm 4; in fractional differencing and cumulation steps we use the FFD and its inverse. We obtain $\widehat{d}$ using $L W$ or $E L W$ with $m=11$ and $m=22$ for $T=120$ and $T=500$, respectively.

Step 4. Then, calculate the statistic of interest and obtain $S_{l, T}^{*}, l=1, \ldots, B$, collecting $S_{B, T}^{*}:=$ $\left(S_{1, T}^{*}, \ldots, S_{B, T}^{*}\right)^{\prime}$. It should be noted here that for $K N, M B B$ and $S B B$ bootstraps, one must use the bootstrap samples as calculated in Step $3 a$ above, inflating $S_{B, T}^{*}$ by $b^{\widehat{d}}$. Then, calculate the $q_{0.05}^{*}$ and $q_{0.95}^{*}$ quantiles of $S_{B, T}^{*}$. Finally, calculate $C_{i}$ which takes the value 1 if $q_{0.05}^{*} \leq S_{T} \leq q_{0.95}^{*}$ and 0 otherwise.

Step 5. Repeat steps (1) to (4) above for all $i=1,2, \ldots, R$ simulations.

Step 6. Report the simulated bootstrap coverage rate for $S_{T}$ defined as $C R:=\frac{\sum_{i=1}^{R} C_{i}}{R}$. 
A.5 Full Set of Simulation Results 


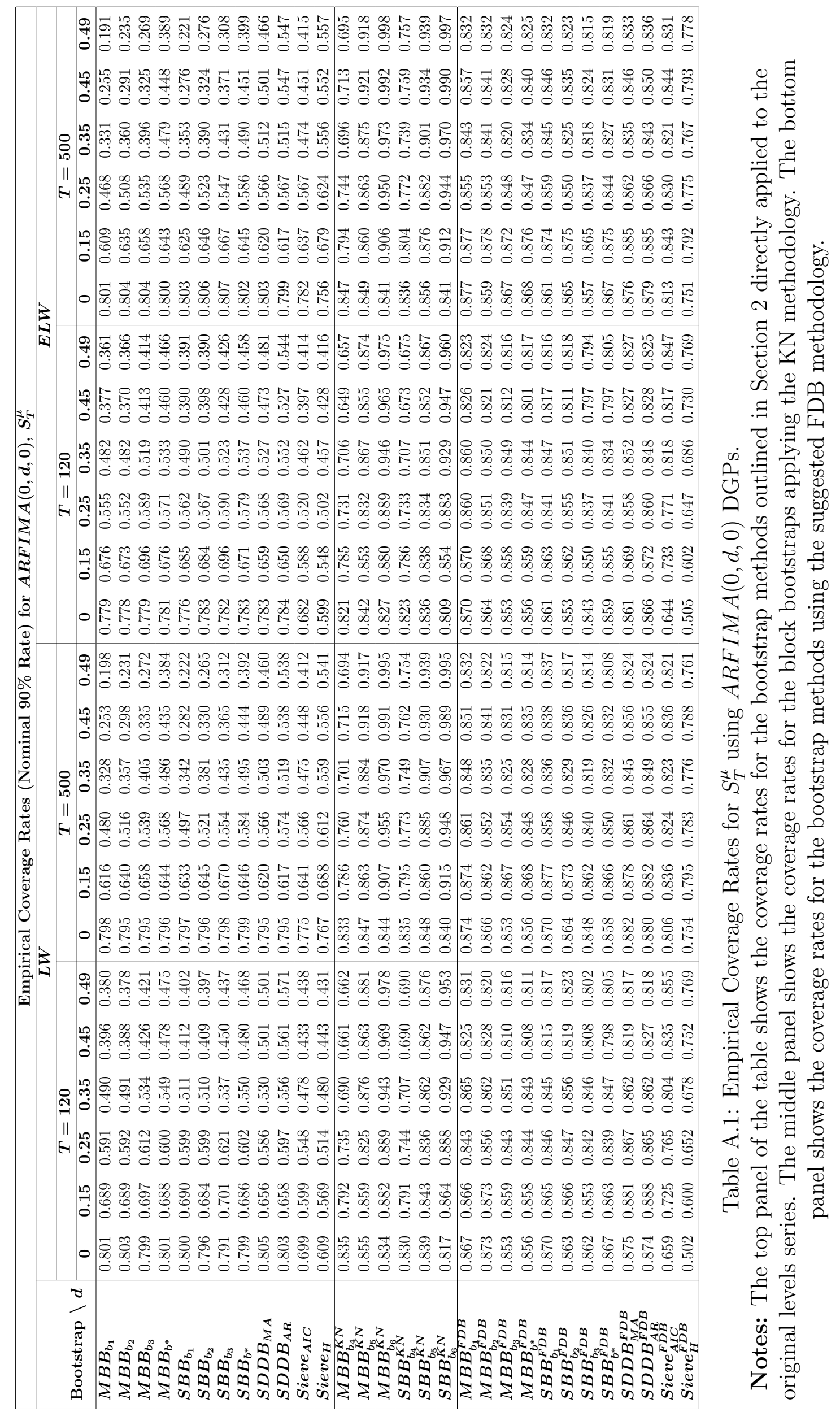




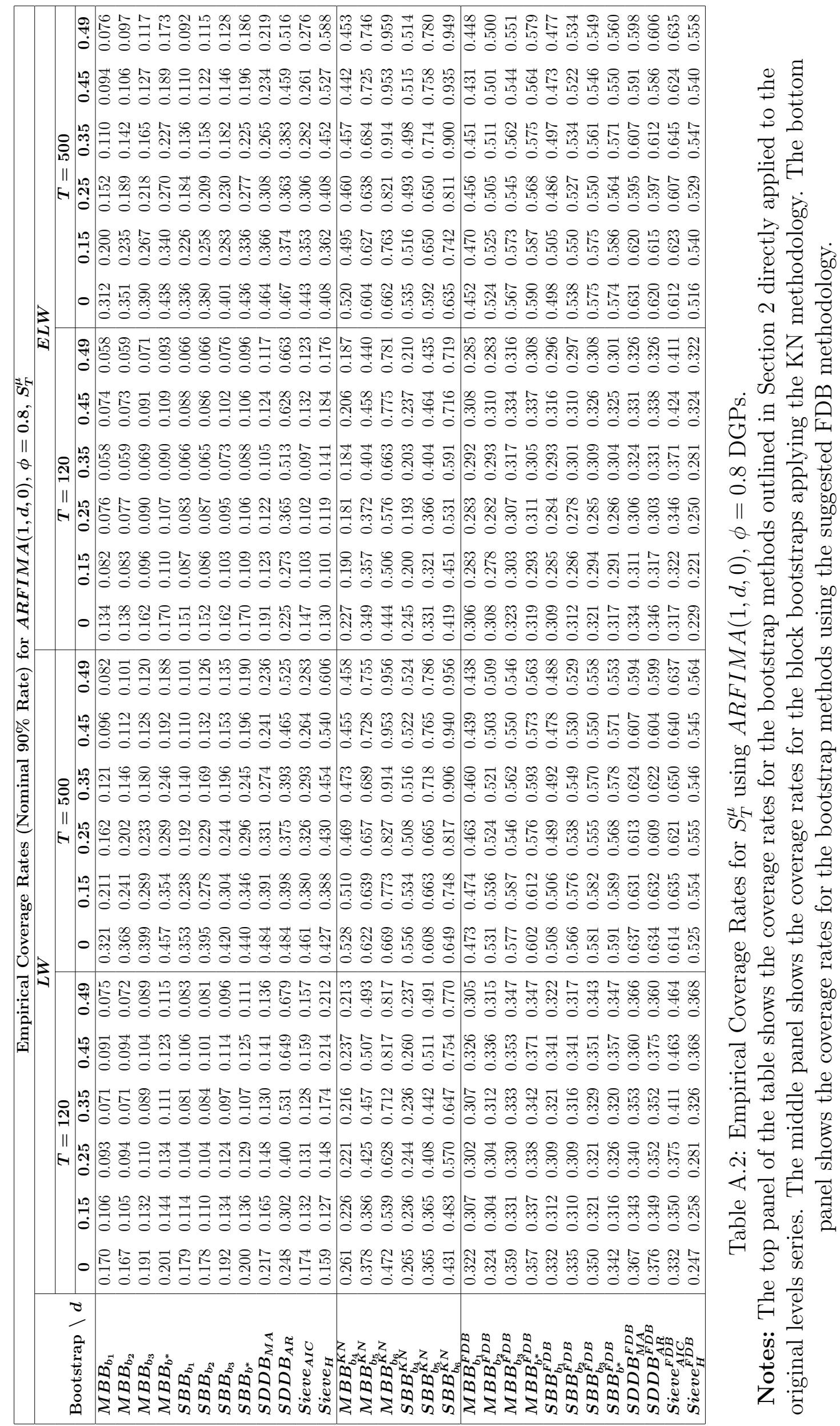









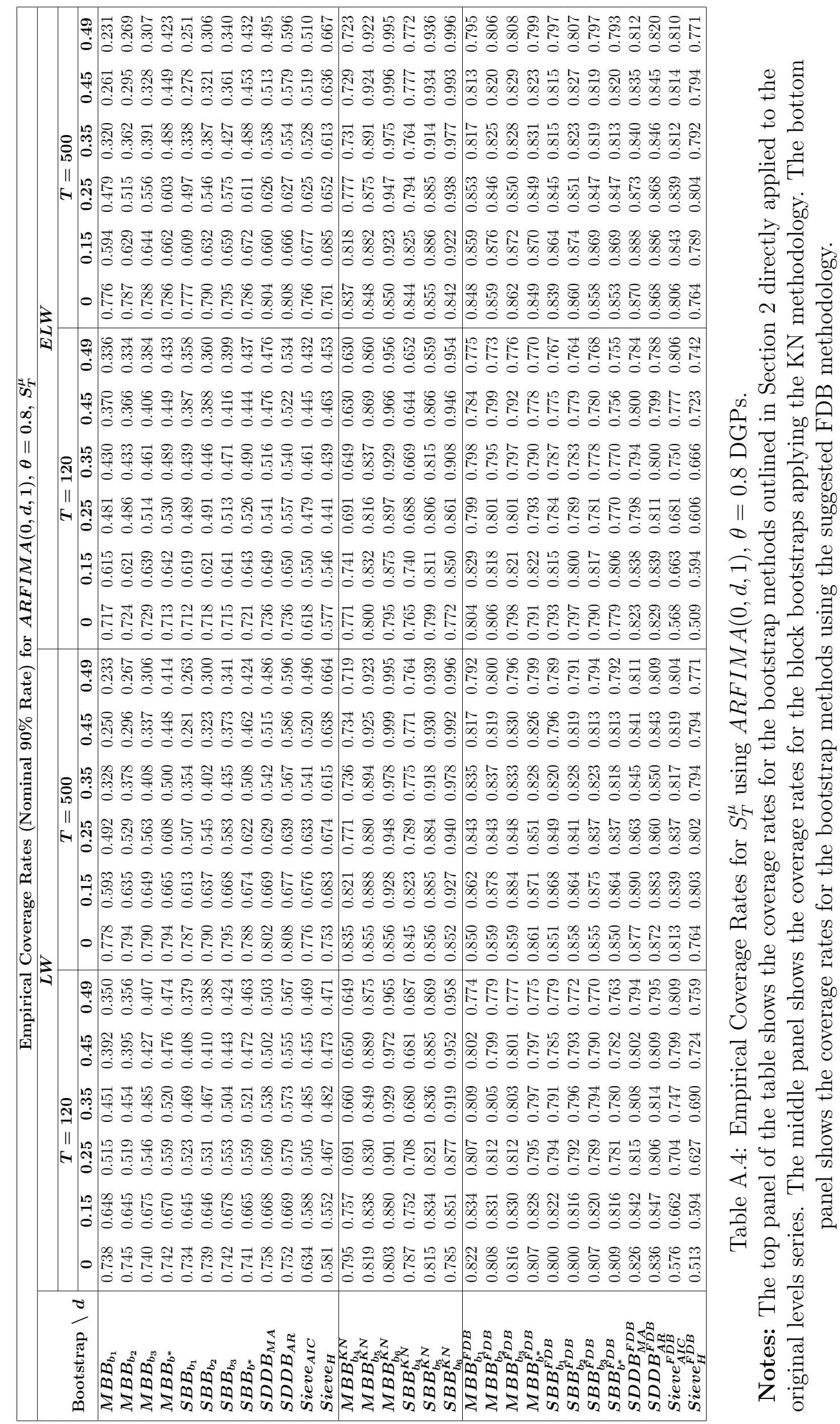




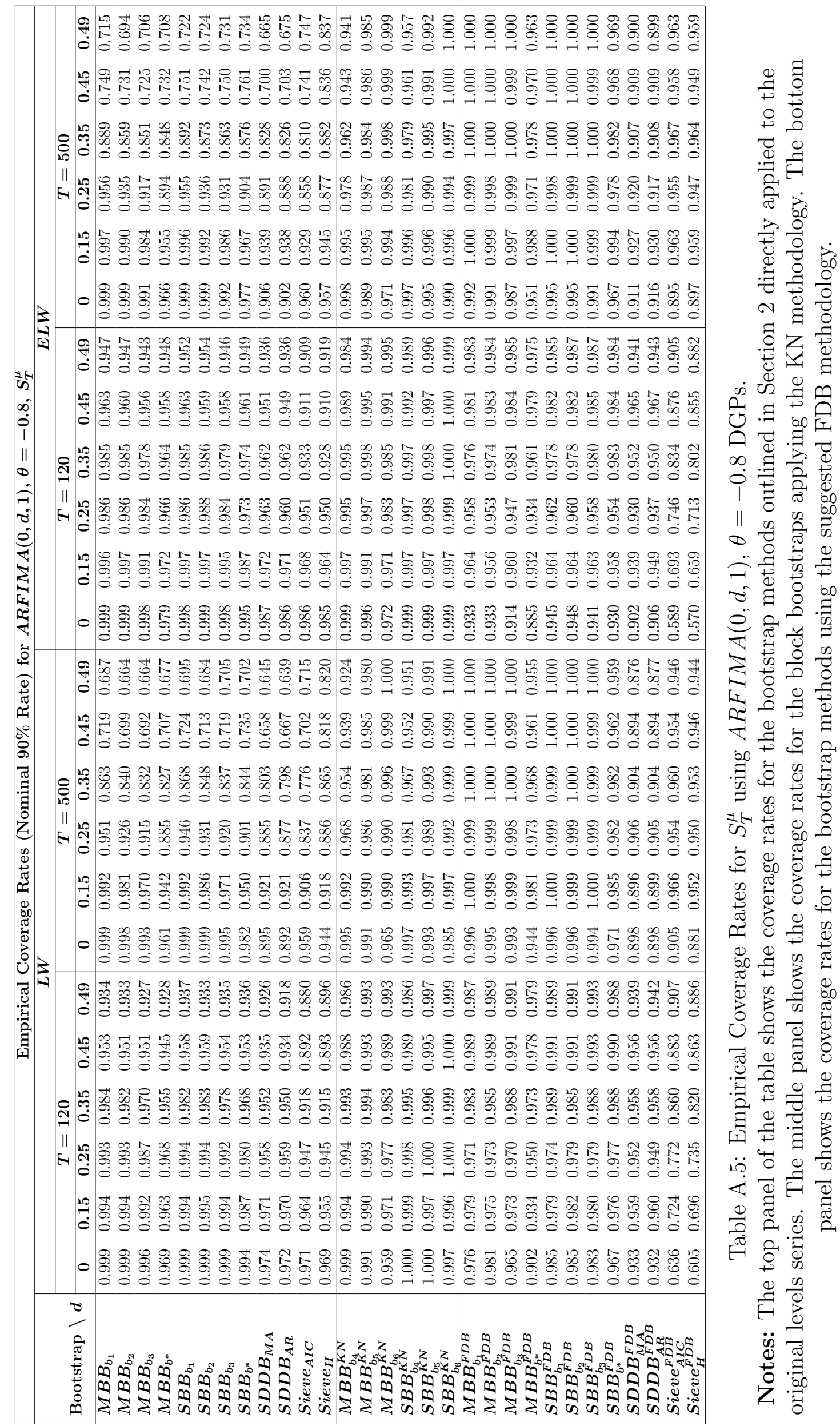




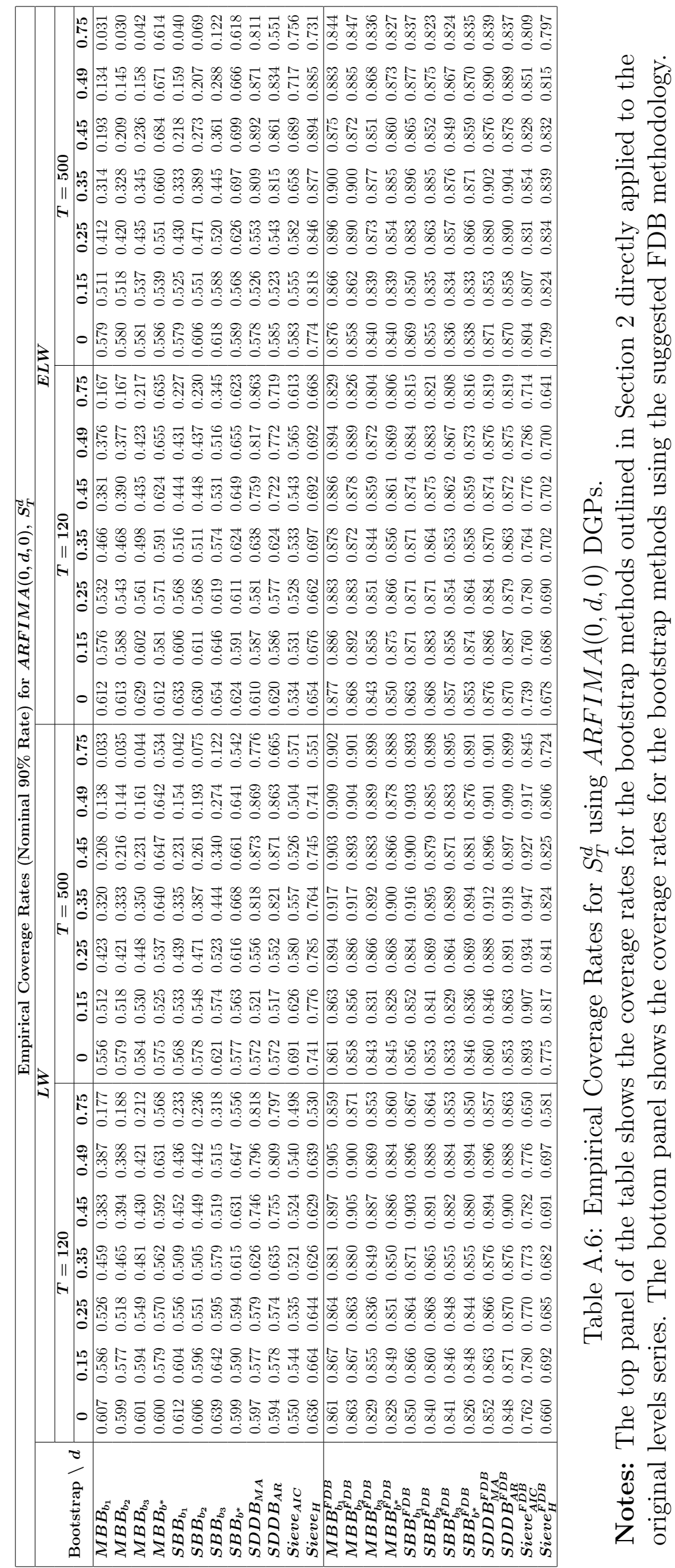




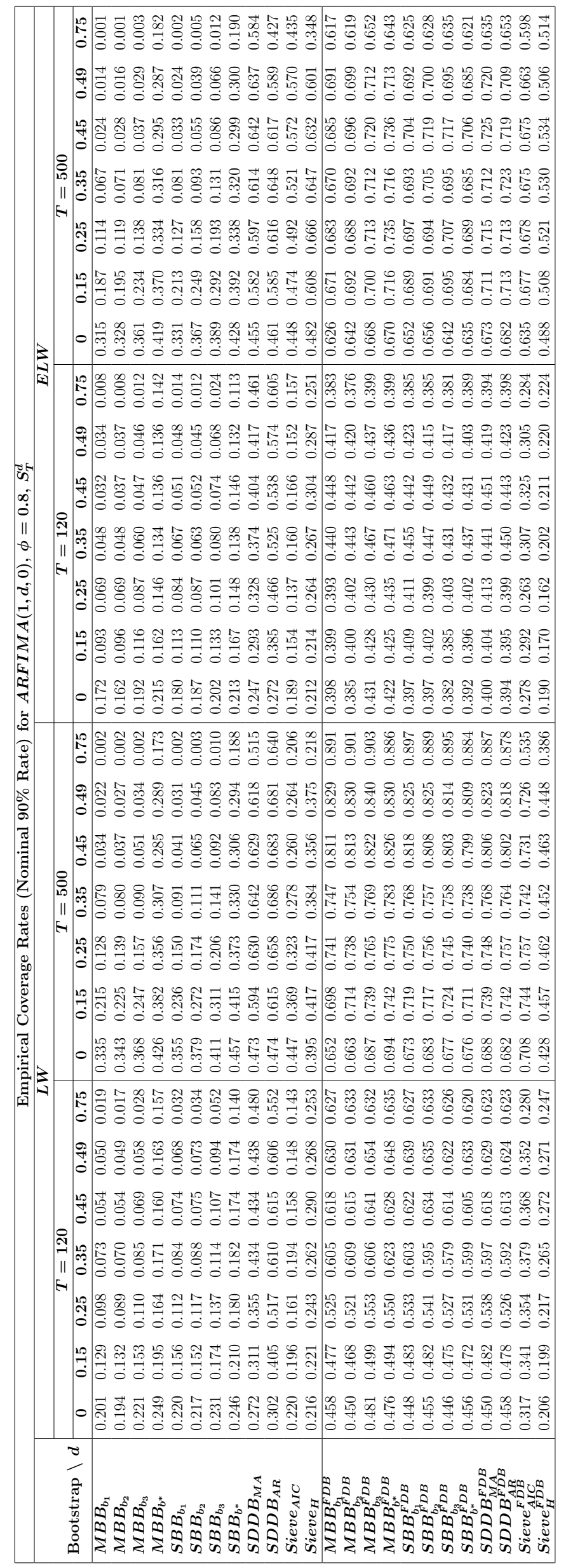

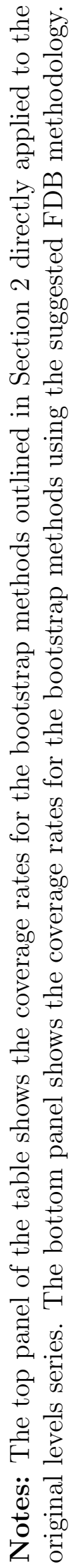




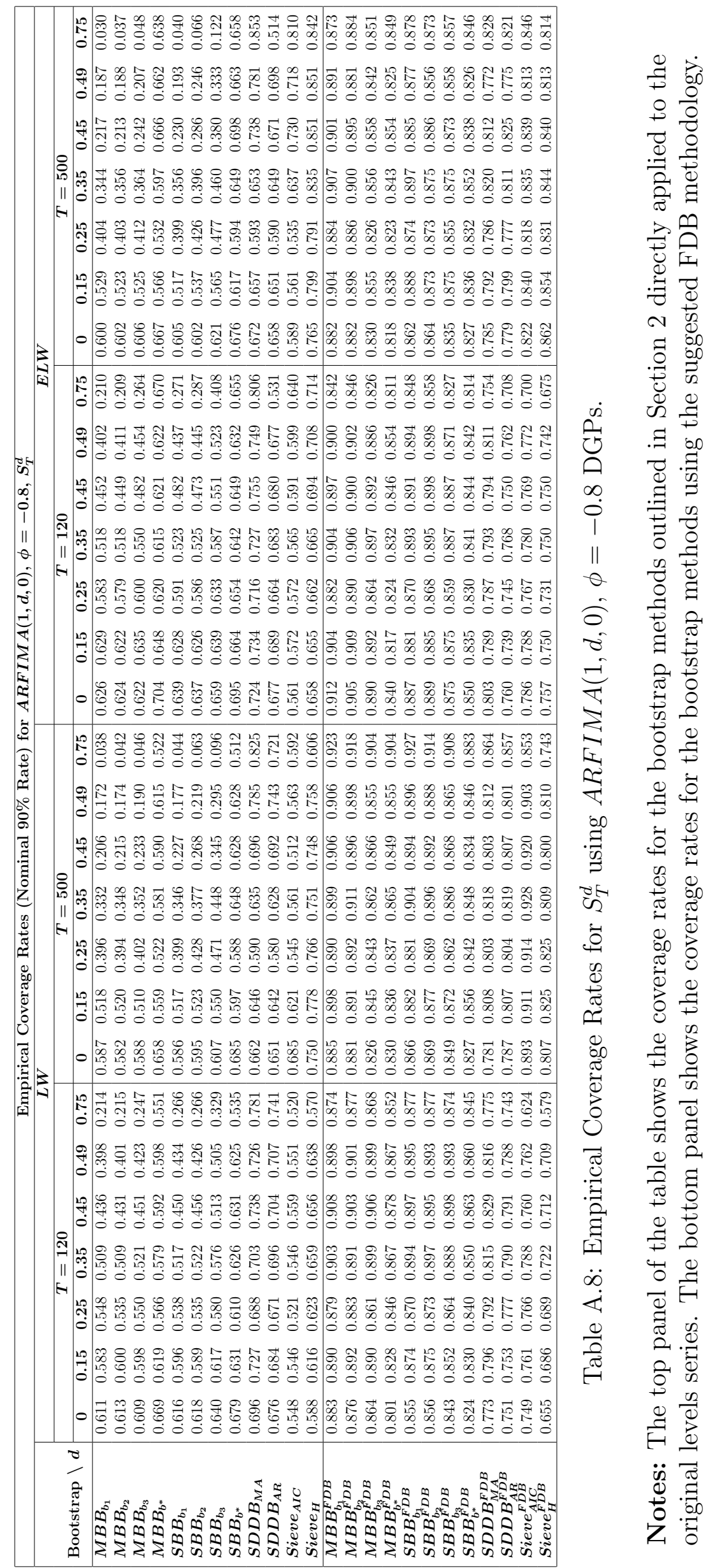




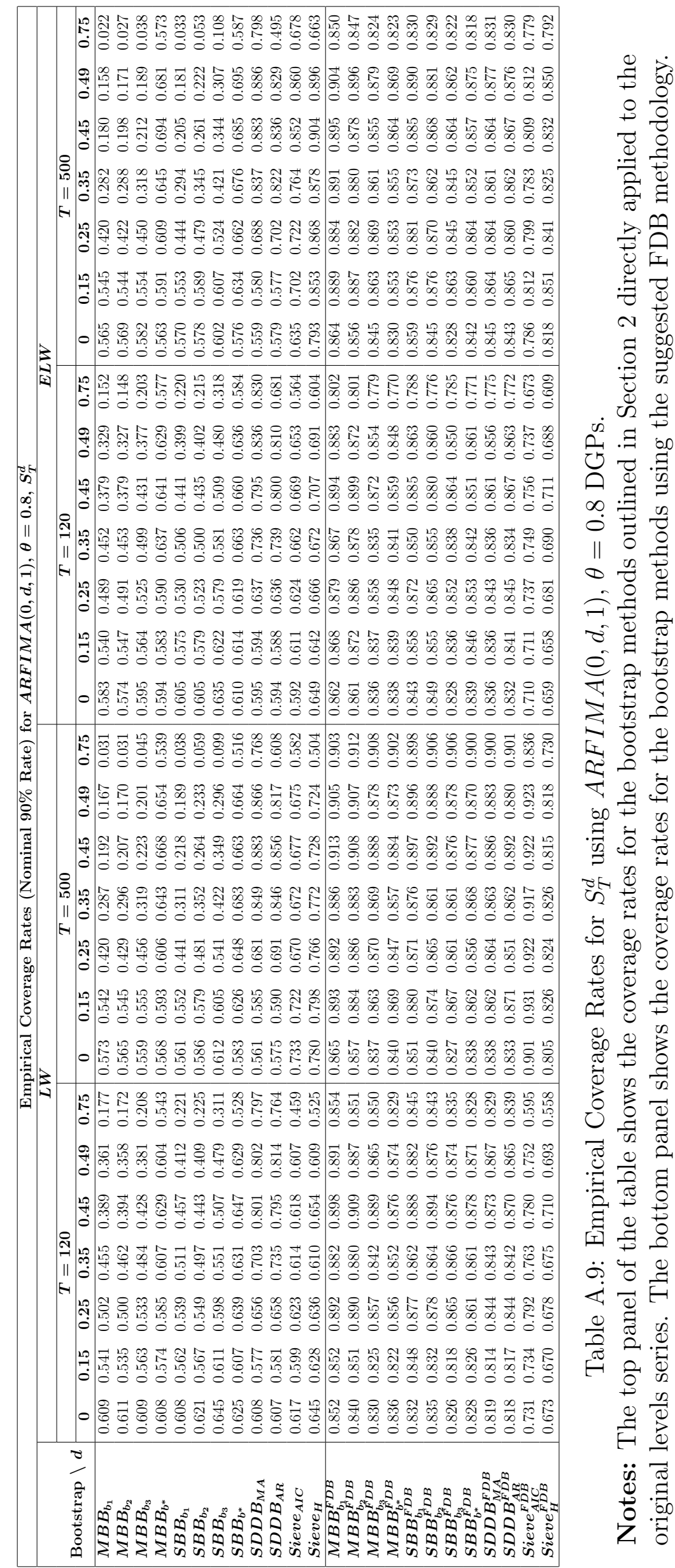




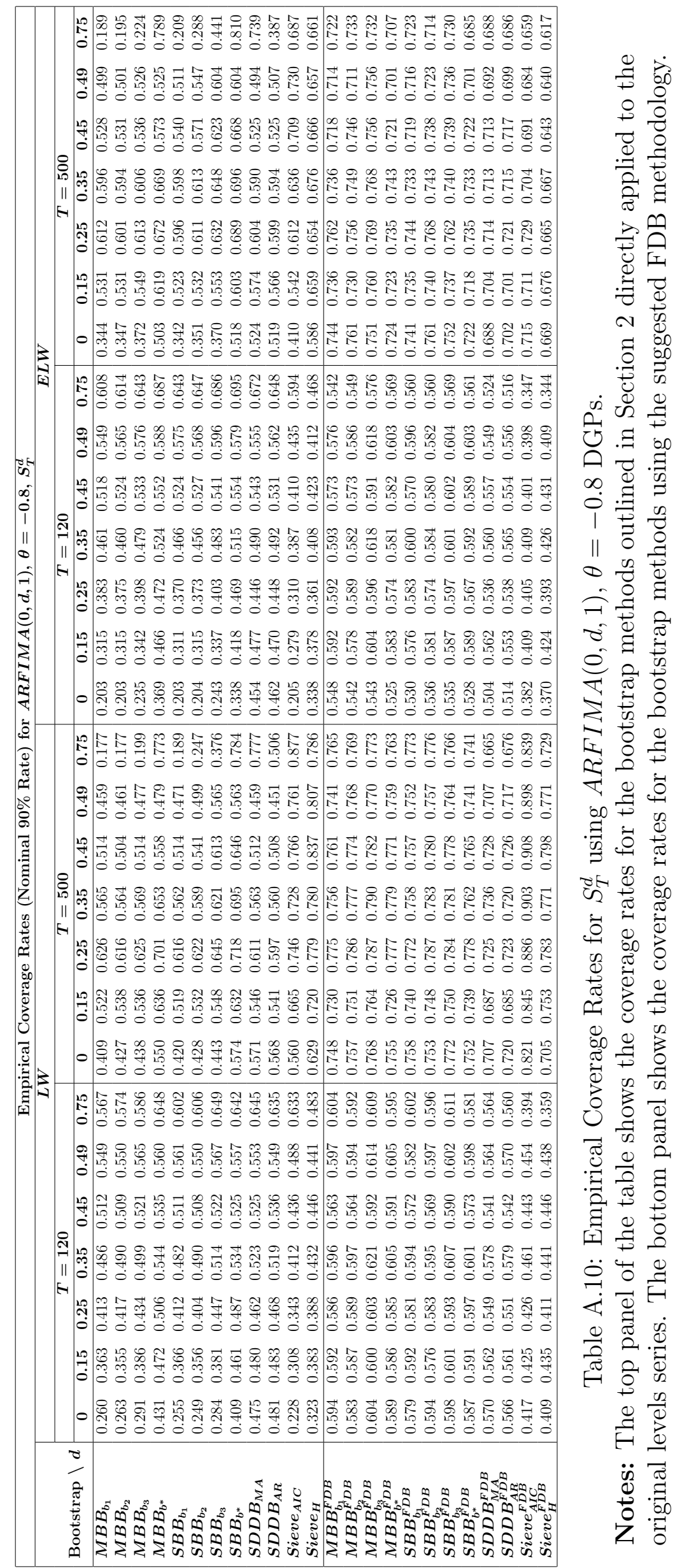




\section{Additional Reference}

Wright, J.H., 1995. Stochastic orders of magnitude associated with two-stage estimators of fractional arima systems. Journal of Time Series Analysis, 16, 119-126. 